\title{
Guidance on the homogenization of climate station data
}

Draft version

November 2018

This publication is a non-peer reviewed preprint

published at EarthArXiv 


\section{Guidance on the homogenisation of climate station data}

Contributions by: Victor Venema ${ }^{1}$, Blair Trewin ${ }^{2}$, Xiaolan Wang ${ }^{3}$, Tamás Szentimrey ${ }^{4,5}$, Monika Lakatos $^{5}$, Enric Aguilar ${ }^{6}$, Ingeborg Auer ${ }^{7}$, Jose A. Guijarro ${ }^{8}$, Matthew Menne ${ }^{9}$, Clara Oria ${ }^{10}$, Wilfrid Serge Raoul Likeba Louamba ${ }^{11}$, Ghulam Rasul ${ }^{12}$

1 Meteorological Institute, University of Bonn, Germany

2 Bureau of Meteorology, Melbourne, Australia

3 Climate Research Division, Science and Technology Branch, Environment and Climate Change Canada, Toronto, Ontario, Canada

4 Varimax Limited Partnership, Budapest, Hungary

5 Hungarian Meteorological Service, Climate Division, Hungary

6 University Rovira i Virgili, Taragona, Spain

7 Zentralanstalt für Meteorologie und Geodynamik (ZAMG), Vienna, Austria

8 State Meteorological Agency (AEMET), Spain

9 NOAA National Center for Environmental Information, Dataset Section, Asheville, North Carolina, USA

10 SENAMHI, Servicio Nacional de Meteorología e Hidrología del Perú, Peru

11 Direction de la météorologie, Brazzaville, Congo

12 Pakistan Meteorological Department, Islamabad, Pakistan 


\section{PART I \\ General topics}




\section{CHAPTER 1 Introduction}

Good quality, homogenised historical data are essential to understand climate variability and change. Climate data analysis cannot be undertaken without a series of preliminary tasks, namely DAta REscue (DARE), which ensures completeness of the datasets, Quality Control (QC), aiming at the identification and removal of erroneous observations and climate time-series homogenisation, which seeks to detect and remove changing systematic biases in the climate time series. However in many countries these historical data to prepare climate assessments are not accessible, or not quality controlled and not homogenised. They cannot fulfil the requirements of Global Framework for Climate Services.

homogenisation, the topic of these guidelines, aims to make data "homogeneous". This word derives from the ancient Greek and means "of the same nature". In the realm of climate science this means that the values of a climate time series must be of the same nature, i.e., comparable. Unfortunately, most long-term raw climate series do not fulfil this principle and are internally inhomogeneous, i.e., not of the same nature and are therefore unsuitable for statistical climate change analysis. Nonclimatic factors such as change in the circumstances of the measurements can cause distortions in the statistical behaviour of the time series. The impact of these distortions can be comparable to that of climate change. This may lead to erroneous conclusions.

Each value arriving at the computer of a climatologist is the result of a series of consecutive procedures: a set of instruments is deployed in a location, the value is measured, recorded, undergoes transformations and is included in a database, and then transmitted to the intermediate and final users. When studying long term series, some of these procedures may have been altered through the years. In a temperature time series starting in the late 19th century, the thermometer has most likely been replaced several times or even substituted by an electronic sensor in recent decades. The shelter has probably changed from an open stand to a Stevenson screen and then possibly to a multi-plate screen if the station has been automatized. Around the mid- $20^{\text {th }}$ century, many observation stations were moved to airports to service the growing demand for civil aviation. If the station remained at the same location, the surroundings have probably been altered. For example, if the station was deployed in the outskirts of a village 100 years ago, today, it might be surrounded by buildings. Perhaps due to land use changes or other practicalities, the station has been relocated to a more convenient place in the suburban area. (More information on causes of inhomogeneities can be found in Chapter 3). If we would combine the observations in such different periods, they would obviously not be comparable. It is rare, although not unknown, for a climate data record of 100 years or more to be truly homogeneous. For example - in the Australian ACORNSAT temperature data set, only 2 out of 112 stations (both starting in the 1940s) were found to be homogeneous for both maximum and minimum temperatures, while in Europe the period between two breaks in temperature observations is about 20 years.

The situation described in the previous paragraph implies that we will not be able to make solid inferences about the temporal evolution (e.g., compute trends) of climate series without ensuring that all the observations and the derived time-series are comparable. No climate time series should be used without homogenisation and all NMHSs and climate data providers should, as part of the core competency for Climate Services "create and deliver climate data sets" routinely conduct homogenisation. 
In this context, homogenisation refers to the handling of long-term issues. Short-term data quality problems (such as an error in a single observation, or an instrument fault affecting a period of a few days or weeks) are considered to fall under data quality control.

Homogenisation is a three-phase process: detection, adjustment and validation. Detection involves analysing the climate series to locate abrupt or gradual inhomogeneities. Detection is performed using statistical tests and visual tools and should be performed consulting metadata, i.e., documentary information on known changes. Adjustment is the process of using statistical approaches to reduce the bias introduced by the inhomogeneities, to make the series comparable.

Both detection and adjustment should be made by comparing the series with neighbouring series. This is called relative homogenisation and relies on the principle that nearby stations share not only the same climate, but also much of the same temporal evolution. If we do not use such comparisons with neighbouring stations (absolute homogenisation) this results in much larger uncertainties of the homogenised series.

The final step is the validation of the homogenised data. No matter how well the data are homogenised, perfection will not be achieved and some residual inhomogeneity will remain in the adjusted series. It is necessary to critically evaluate the work and to review the homogenised data. This review should look at individual series and assess whether the new values make sense: is the seasonal cycle preserved, are the values in the expected range for the station, etc. If we have homogenised a full dataset, looking at the regional coherence of the temporal evolution of the series is extremely useful, as well as comparing the adjustment series to the known changes in the network.

For selecting appropriate homogenisation methods we must rely on their design principles and on validation and benchmarking studies using simulated series to assess their capabilities; see Chapter 4 for details. These evaluations can inform the operator which method is suited for the task at hand, which will vary according to the variable to be homogenised (e.g., temperature, rainfall, pressure, etc.), the data resolution (e.g., daily, monthly), the number of series to be homogenised and their spatial cross correlations.

There is a rising awareness that homogenised data is not a final product that can be analysed as if it were homogeneous, but that climatologists should rather see homogenisation as part of the data analysis. The analysis of climate data should, for example, take into account that the homogeneity of homogenised data depends on the station and the period considered. An urban climatologist needs to homogenize data differently (Zhang et al., 2013), than a climatologist interested in global warming or land-use change. Reversely the understanding of climate variability can help in producing more accurate homogenised data (Wen et al., 2010).

\subsection{Scope of this report}

This guidance was prepared by the Task Team on homogenisation (TT-HOM) of the Commission for Climatology (CCl). TT-HOM is part of the $\mathrm{CCl}$ Open Panel of $\mathrm{CCl}$ Experts on Climate Monitoring and Assessment (OPACE-2). The report aims to give guidance to users and developers of homogenisation methods for climate station time series.

There are two fundamental types of homogenisation: homogenisation of the annual, seasonal or monthly means, and homogenisation of the distribution that also adjusts the variability around the mean. The focus of this guidance will be on the homogenisation of the means; this type is understood best and most commonly applied in operational practise. Adjustments of the distribution are important for studies on changes in weather variability and severe weather. This guidance will 
explain the limitations of only homogenizing the means, but the adjustment of the distribution is difficult and not sufficiently well understood at this time. This is thus beyond the scope of this guidance.

Climatic datasets of any kind typically contain inhomogeneities. This guidance will be limited, however, to the homogenisation of instrumental land station data. Many lessons will carry over to the homogenisation of radiosonde data (Jovanovic et al., 2017, Haimberger et al., 2012) and other types of data. For more information on the homogenisation of marine in-situ data see Kent et al. (2016), Kennedy et al. (2011) and Huang et al. (2015). Some papers on biases and homogenisation of satellite data are Schröder et al. (2016) and Brogniez et al. (2016).

Part I of this report, chapters 1 to 4, is aimed at getting new people started with homogenisation. Chapter 2 discusses the prerequisites and why they are important for homogenisation: data rescue of the observations themselves and of information on the history of the measurements, as well as quality control (QC).

Chapter 3 is the main chapter about best practises for homogenisation. It lists examples of reasons for inhomogeneities to illustrate the kind of problems a homogenizer may encounter and explains the various approaches to homogenisation and how to select the data you need. The selection of adequate homogenisation software for the task at hand is the topic of Chapter 4. It discusses the publicly available software packages, their strengths and weaknesses and the criteria to use for the selection. People starting with homogenisation are nowadays well advised to use well-tested existing software rather than developing their own. This list of software packages will be kept up to date on the internet.

Part II of this report discusses more advanced and background topics and is intended for advanced users and developers of homogenisation methods. Chapter 5 gives a short historical overview of homogenisation methods. A mathematical description of the homogenisation problems can be found in Chapter 6, which is aimed at designers of homogenisation algorithms. Writing such a guidance also makes clear what we do not know or not well enough yet. Thus finally in the last chapter ideas for future homogenisation research are given.

This report is accompanied by a frequently asked questions page (FAQ) in the internet. These questions can be of a more practical nature or more transient nature (for example, software bugs and solution for them). 


\section{CHAPTER 2}

\section{Prerequisites}

Homogenisation is one step in the processing of climate data. Previous steps influence the quality of the homogenisation and this should be taking into account in the subsequent climate data analysis. These previous steps include DAta REscue (DARE; Section 2.1), Quality Control (QC; Section 2.2) and network selection (sections 3.6 and 3.7).

Network selection is discussed at the end of Chapter 3 on the homogenisation practise because this requires knowledge of homogenisation; also data rescue and quality control are aided by a good understanding of homogenisation. For this chapter the main thing to know is the importance of the signal to noise ratio for quality control and statistical homogenisation. Data rescue is thus also important in regions and periods where we do have data, but the station density is not yet sufficient to see important data problems.

Before homogenising a dataset it is important to know how the variable was measured historically throughout the network and what happened with the stations. Over the course of homogenisation process, awareness of the volume of the missing data is essential. If the volume of missing data crosses a threshold over a period of time some homogenisation approaches may not work as expected. Also during homogenisation metadata is important to validate the results of statistical homogenisation and to understand what happened in complicated situations. The
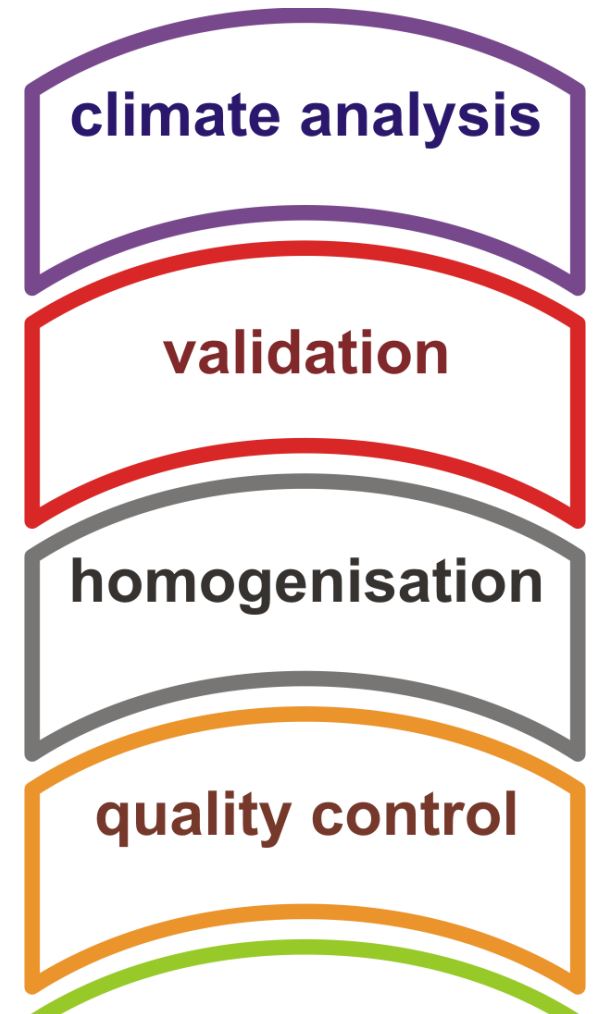

network selection

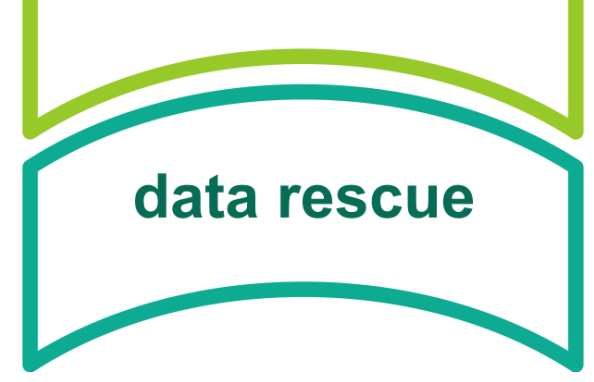
role of metadata and its quality is discussed in Section 2.3.

The final section highlights the importance of training and gives pointers to the main scientific meetings on homogenisation.

\subsection{Data rescue}

Many observations are still not available digitally, especially in the case of daily station data. Whilst considerable effort has been devoted to data rescue in recent years in various parts of the world, a great deal remains to be done.

Data rescue itself is a multi-faceted challenge, including locating and preserving manuscript records, and making the information contained in them available in a digital form. Detailed discussion of the practical aspects of data rescue is outside the scope of this document. The WMO Commission for Climatology has an Expert Team on Data Rescue (ET-DARE) ${ }^{1}$, and published Guidelines on Climate

\footnotetext{
${ }^{1}$ The ET-DARE website is located at http://www.wmo.int/pages/prog/wcp/ccl/opace/opace1/ETDARE-1-2.php.
} 
Data Rescue in 2004 (WMO, 2004), although there have been developments in the field since then, especially in areas such as the use of crowdsourcing for digitization.

Data rescue plays two important roles in the development of homogenised climate data sets. The first and most obvious is that a data set will not be available to be homogenised and analysed unless it is available in digital form. Less obviously, homogenisation is most effective when a candidate station can be compared with a large number of reference stations in the region (see Section 3.6), something which requires digitized data from those reference stations as well as the candidate station. Reference stations are also useful in quality control, as are sub-daily (e.g., hourly) observations at the candidate station. Sometimes it will be known what undigitized data exist in a country or region (for example, at stations where monthly data are digitized but daily data are not), but sometimes it is not known where observations were made, and/or the physical location of the written records.

Sections 3.4 on break detection and 3.6 on comparison series expound the importance of the signal to noise ratio (SNR) for homogenisation. Defining the SNR as the variance of the break signal divided by the variance of the noise it is important that the SNR is above one. As long as this level is not yet reached further data rescue for this period and region should be prioritised, providing further undigitized data exist. Digitising clusters close to a candidate station of interest is preferable to a uniform sampling when it comes to data quality control and homogenisation. The digitisation of short series can also be worthwhile where they can help QC and homogenisation.

\subsection{Quality Control}

Quality control (QC) aims to detect data values that are obviously in error. Apart from affecting the value of a dataset in its own right, this is necessary because large outliers can influence homogenisation detection and correction and subsequent climate analysis. Data quality control is especially important when dealing with weather variability and extremes - both the removal of errors which appear to be extremes, and ensuring that genuine extremes are not removed during quality control processes.

Since large outliers can affect the homogenisation process, QC should be performed before homogenisation. Sometimes QC is performed again after homogenisation because the higher data quality allows the detection of more subtle erroneous values. The interaction between $Q C$ and homogenisation is not strong, however, and mostly this is not necessary.

Quality control can also be a source of inhomogeneities, especially in daily data when analysed for changes in weather variability and extremes. The methods used for QC of the data have often changed over time and this can introduce inhomogeneities if, for example, erroneous data were not being detected in the past but are now being flagged. In many cases older historical data received very limited QC; in particular, QC methods which involve spatial intercomparison of data with other stations have only been practical since the introduction of modern computer systems. More recently, the level of manual intervention in $Q C$ is being reduced in many countries, and whereas in the recent past, a data point flagged by an automatic system might be subject to manual review, increasingly QC is purely automatic, sometimes leading to an increased risk of 'false positives' where valid extremes are flagged as suspect.

Also when the (recent) data was subjected to real-time $Q C$ an additional climatological $Q C$ is necessary. Data that was not yet available in real time may help find errors and during digitisation and collating data new errors can be introduced and a climatological QC offers a standard minimum quality over the full period reducing the influences of inhomogeneities in quality. Furthermore, 
independent QC (in parallel to any QC applied by the original data custodian), is important for achieving an as uniform as possible data quality across the full period of record (although this will not make the QC fully uniform, if only because reference data from nearby stations are likely to be more sparse in the earlier part of the record).

Derived time series can have quality control problems that were not obvious in the single observations. It is desirable to carry out $Q C$ at a range of timescales to detect the full range of possible error modes. A observation or clerical error (for example, a misplaced decimal point or misreading a thermometer by 10 degrees) is likely to affect only a single observation. Other errors such as instrument faults may be too small to be detectable on any individual day, but may be detectable in monthly data with its lower level of noise (and hence higher signal-to-noise ratio).

Quality control is about detecting observational errors. Homogenisation is about making data more comparable, which can be, but does not have to be, because of subtle errors. These are two fundamentally different problems. There are, however, sometimes mixed cases that could belong to both categories. For example, when a measurement problem occurs and is fixed after a time this produces a measurement problem that depending on the length can be seen as a QC problem or as a platform-like break pair. Due to errors in digitisation (minus signs missing, multiplication factors not implemented), collation/merging (stations with a similar name mixed up) and observers not following procedures over a long time (not measuring overflow of rain gauge, reading wrong side of minimum-maximum thermometer) clearly wrong values can also persist over periods typically seen as homogenisation time scales.

Some homogenisation software includes a quality control component, but in general it is preferable if quality control is performed before the homogenisation process starts.

Discussion of specific quality control tests and methods is outside the scope of this publication. For a fuller discussion of quality control, readers are referred to WMO (1993) and for a good example to Dunn et al., (2012).

While homogenisation procedures often include missing data completion, longer contiguous missing data periods can obstruct the homogenisation process. This is especially a problem in case of sparse networks when multiple stations in a region are incomplete simultaneously. Note that the QC, the missing data completion, the detection of inhomogeneities and adjustment of the series are closely related problems. Generally the output of a successful homogenisation process are complete, quality controlled and homogeneous data series.

\subsection{Metadata \& station history}

\subsubsection{What is metadata?}

In the context of homogenisation, the term 'metadata' is used to refer to what, in some other contexts, is called 'station metadata', important for homogenisation is the 'station history'. This includes information about the observation site and instruments, and observation and dataprocessing procedures. These can include (but is not limited to):

- The location and elevation of a station.

- The types and conditions of instruments used at a location.

- The land use and vegetation type in the vicinity of a station.

- The standard times and frequency at which observations are made.

- The name of the observer(s) (for a manual station).

- Procedures for processing data (for example, the definition of daily mean temperature).

- Results of calibrations or tolerance checks carried out at a station. 
- Details of maintenance (scheduled or unscheduled) carried out at a station.

Metadata includes both the status of a site at any given point in time, and information throughout a site's history.

Metadata can exist in a wide variety of forms. In many national meteorological services, some or all recent metadata is held in digital form within a searchable database, but large amounts of historical metadata normally exist only on paper. Metadata may exist in forms specific to an individual station, or documents that cover a large number of stations (for example, observation procedures often apply to a whole national network and are covered by national-level documents rather than stationspecific ones). In many countries, stations are inspected regularly by network management staff, and these inspection reports form a significant part of available metadata. Sometimes an observer, their family and newspapers, financial accounting or other external documents can provide additional metadata.

\subsubsection{The value of metadata, and its limitations}

Metadata, where it exists, is an extremely valuable part of data homogenisation. While statistical methods for detecting inhomogeneities can provide strong evidence that an inhomogeneity can occur, metadata can indicate the cause of an inhomogeneity, and can also (if recorded) determine the date of an inhomogeneity with high precision, whereas statistical methods can only determine the timing with limited precision (typically within a few months to a year). Metadata can also provide evidence of inhomogeneities in situations where statistical methods have limited or no effectiveness - for example, where there are no nearby reference stations, or where a change affects a large part of the network at the same time.

There is no consensus on whether metadata should influence whether a break found by statistical homogenisation is set or not. This is potentially dangerous because not all typical breaks are equally well documented. The best-known example would be an urban station where the gradual urbanization is typically not well documented while the relocations are better documented. It would make the trend errors larger to only remove the documented relocations. On the other hand, with several breaks in the candidate and reference series, homogenisation can be a difficult combinatorial problem. In such cases, known likely breaks can help solve this puzzle.

Metadata is most valuable where it is complete. Many national meteorological services have good metadata in recent years, but its availability often becomes sparser as one goes further back in history (and the older metadata is more likely to be on paper, which can be difficult to locate or access). As most stations, particularly prior to the 1990s, were installed to support weather forecasting rather than for climate purposes, recording metadata that was not relevant to a weather forecasting function was often not a high priority. Also, sometimes metadata reports provide a snapshot in time and cannot demonstrate the date of a change (for example, two station inspection reports five years apart may show the station in different places, but there may be no documentation of exactly when the move took place).

Some aspects of metadata are also better-documented than others. Experience shows that new instruments are often well-documented, as their installation normally involves the expenditure of public funds, something which is rigorously documented in many countries. On the other hand, documentation of the local site environment, especially outside the immediate vicinity of the instrument enclosure, is often limited or non-existent. Metadata may also be more limited in situations where the national meteorological service uses data provided from stations installed and operated by third parties, rather than by the national meteorological service itself. 
For these reasons, homogenisation is normally most effective when it uses a combination of metadata and statistical methods. In addition to being used directly, metadata can sometimes be used in support of adjustments made by statistical methods - for example, if an inhomogeneity is found statistically, metadata may provide an explanation for this even if the inhomogeneity was not revealed by an initial search of metadata. One example of this was at Victoria River Downs, a site in a savanna climate in the Northern Territory in Australia. Statistical testing found an inhomogeneity in 2007 of approximately $+1^{\circ} \mathrm{C}$ in maximum temperatures during the dry season; an inspection of station photographs, which had been taken annually, then revealed that the grass around the observation site had been irrigated up until 2007, but after that was allowed to die during the dry season.

Some forms of metadata will be more relevant for some variables than others. Changes of observer are potentially highly relevant for variables that require observer judgment, such as cloud amount, and may signal relocations in voluntary networks, but are less relevant for instrumental variables in professional networks such as temperature; land surface type may be important for temperature, but is of limited significance for precipitation.

\subsubsection{Formats, interpretation and uncertainty of metadata}

Metadata can take the form of a single point of data (for example, a set of station coordinates) or a more complex piece of information (for example, a photo of the station which provides information on the land surface type and surrounding obstructions).

Even the more simple forms of metadata have some level of uncertainty attached. Until recent years, station coordinates were rarely recorded sufficiently accurately to resolve small site moves (in the tens of metres), but such moves may still be climatically significant, especially in complex topography or where they affect the site's exposure to wind. (In exposed coastal locations, site moves of less than 50 metres have been found to have a $15-25 \%$ impact on observed precipitation). In older datasets, or those exchanged internationally, station coordinates are often only reported to the nearest minute or 0.01 degree, which only resolves the location of the station to within about 2 kilometres. Thus a change in the coordinates could also be a new determination of the coordinates using improved technology (e.g., GPS).

More complex forms of metadata often require considerable interpretation. At the most complex end of the scale, it may be necessary to use multiple pieces of metadata information to reach a conclusion - for example, a site move may not be specifically documented, but there may be a number of items of information relating to the site at an earlier date that are not consistent with its being in its current location (for example, descriptions of local topography or references to nearby roads or buildings). Such information is often not amenable to being placed in a standardised format for inclusion within a database, one of the barriers to work currently in progress in WMO to develop a standard digital format for metadata.

One challenge when dealing with metadata documents is identifying what information is important. Information about relevant changes will often be filed together with other correspondence related to the station of no climatic relevance (such as financial documents), and separating relevant from nonrelevant documents can be a time-consuming process.

As with data, metadata can also sometimes be in error, making it useful to have multiple lines of evidence for an inhomogeneity where that is possible. 


\subsubsection{Station identifiers}

Determining exactly which station a dataset is associated with is an important part of developing long-term homogeneous datasets. In most countries, a station will have a domestic station identifier, with stations which report internationally also having a WMO station identifier. It may happen that two or more stations share the same WMO station identifier. There may also be other identifiers in use, such as a station name, or an ICAO code for an airport site.

Best practice is to associate a station identifier with a station, and if that station undergoes a climatically significant change (for example, a significant site move), to cease recording data under that identifier and create a new identifier for the new location. This maximises the visibility of the change, while still allowing a long-term dataset to be created by using a composite of multiple identifiers. (Multiple identifiers are, of course, a necessity if parallel observations are taking place, which is also recommended).

It is, however, common practice to retain a single station identifier even through the process of a significant change. This is particularly true for historical data when awareness of the climatic impact of site changes was lower than it is now, but is still a regular occurrence now, often because the convenience of being able to continue to report for a major location (e.g. a city) under a single identifier is seen to outweigh the arguments for a changed identifier. As such, while station identifiers are a very useful form of metadata, it cannot be assumed that the use of a single identifier in a time series is an indicator of homogeneous data.

Note that the WMO station identifier is not a unique station identifier in some national archives. The WMO station identifier was initially for weather forecast purposes and its number of digits limited. Two or more stations can thus share the same WMO station identifier. In addition new stations sometimes re-use the identifier of a closed station; there are also cases where national identifiers have been re-used.

International datasets face particular challenges in identifying stations. Typically, such datasets will contain data from multiple sources which may be partial duplicates of each other, with metadata often limited to station coordinates (possibly of limited precision) and a station name (which may have multiple possible spellings, especially if the original name is in a language which does not use the Roman alphabet). Rennie et al. (2014) detail the procedures used to merge data from multiple sources in one major international data set, the International Surface Temperature Initiative (ISTI) databank.

\subsubsection{Accessibility of metadata}

Obtaining access to metadata is often a significant challenge. As noted in section 3.3.2 above, substantial quantities of metadata have not been digitised and are only available in paper form. Most metadata is specific to individual locations and may be locatable in files indexed against that location. Other relevant metadata, especially that dealing with network-wide standards and changes, may be in annual reports or other documents which may be more difficult to trace. The use of paper documents may only be possible through visiting an archive location in person. 
Metadata, other than the most basic (such as station coordinates), are not normally exchanged internationally, although a WIGOS metadata standard has just been published, which will allow for this to occur (WMO, 2017). Access to metadata may also be limited, especially for external researchers, because files containing metadata may also contain information (e.g. financial information, or personal information about observers) to which access is restricted.

Finally, metadata is normally only available in the local language.

\subsection{Training and exchange}

Next to data, metadata and methods also training of the operator is important for the quality of homogenised data. Training is important for multiple reasons: A good grasp of the general concepts, an understanding of the statistical background of the homogenisation process, and selection of the best methods and for handling the selected software. Moreover the evaluation of the results requires a trained expert. The availability of training and of local support are conversely also considerations in the selection of homogenisation methods.

Every year Meteo France organises a two week training "Climatology, foundation for climate services" with 3 days about homogenisation and indices. The Universitat Rovira i Virgili (URV) in Tarragona, Spain, occasionally offers trainings at the request of the WMO.

The main meetings to keep up to date with and contribute to homogenisation research are:

- The Seminars for homogenisation and Quality Control in Climatological Databases in Budapest, Hungary.

- The Data Management Workshops of EUMETNET.

- The yearly session on "Climate monitoring; data rescue, management, quality and homogenization" at the Annual Meeting of the European Meteorological Society.

- The yearly session on "Development of climate datasets: homogenization, trends, variability and extremes, including sub-daily timescales" at the General Assembly of the European Geophysical Society.

- The session on "Climate data homogenization and climate trends/variability assessment" at the International Meeting on Statistical Climatology.

The history of the homogenisation of climate data can be followed by the series of the Seminars for homogenisation and Quality Control in Climatological Databases organized by the Hungarian Meteorological Service regularly from 1996. The most proceedings of the Seminars are published through the World Climate Data and Monitoring Programme (WCDMP) series (http://www.wmo.int/pages/prog/wcp/wcdmp/series.php9, namely WCDMP-41, WCDMP-56, WCDMP-71, WCDMP-75 and WCDMP-78). The homogenisation Seminars promoted the discussion of homogenisation methods, with emphasis on their theoretical aspects, practical applications and evaluation of methods. The homogenisation Seminars in Budapest is the origin of the COST-ES0601 Advances in homogenisation methods of climate series: an integrated approach - "HOME" Action ". Past and current efforts in this subject can be found on the website of the "HOME" initiative: http://homogenisation.org/. 


\section{CHAPTER 3}

\section{Homogenisation practise}

\subsection{Introduction}

The purpose of this chapter is to describe general issues related to the development of homogenised datasets. Nowadays, homogenisation is normally best performed with well-tested existing software if available; specific software packages for data homogenisation are discussed separately in Chapter 4 .

There are many factors that can cause inhomogeneities in a climate record. Some will affect only a few climate elements at any specific location, some only affect a single location, while others may affect an entire observation network or substantial parts of it. The latter can cause large-scale biases and are thus climatologically especially important and they are difficult to remove by statistical homogenisation if they happen over a short period. Inhomogeneities may have a seasonal cycle and/or be dependent on weather type (i.e., regime-dependent).

Some examples of causes of inhomogeneities include:

- A site move. For example, early stations often started in towns and villages and were often moved to outlying areas or airports. Early automatic weather stations sometimes had to be placed close to buildings (Menne et al., 2010), while with modern technology it has become easier to place station in pristine locations or move them from the middle of a village to outlying areas (Linden et al., 2015).

- A change in the local environment. Examples of changes in micro-siting are growing or cutting vegetation around a site, or the construction or removal of a building nearby. Also starting to water the grass below the instruments may produce an inhomogeneity. Early temperature and precipitation measurements were often performed at a height of several meters, while nowadays 1.5 to 2 meters is standard. Development of the larger environment (urbanization) can cause a gradual local warming and often provokes a site move, which would likely be a cooling drop. Changes in irrigation practices in the region may lead to artificial cooling (Cook et al., 2014). Moving an anemometer from the roof of an airport to the standard $10 \mathrm{~m}$ level often introduce a big drop in surface wind speed (Wan et al., 2010).

- A change in instruments. For precipitation changes in the outside geometry and windshield will change the characteristics of undercatch and thus produce inhomogeneities. Changes in gauge/instrument type will change typical measurement errors such as the wetting loss and change measuring precision (Wang et al., 2017, Wang et al., 2010). For temperature changes in the screen and thus in the radiation and wetting protection of the thermometer are especially important. Even the position of the thermometer within the screen can matter. There was one report of a plastic Stevenson screen letting the sun in on hot days. Mechanical ventilation can reduce radiation errors, but also produce stronger wetting errors and it may perturb the stable boundary layer. The response time of modern thermometers and their small screens is intrinsically much shorter, which is especially important for the daily minimum and maximum temperature due to turbulent fluctuations. The glass of some early thermometers shrunk in the first years (Winkler, 2009). Calibration errors can cause inhomogeneities. Quicksilver thermometers cannot record temperatures below $-39^{\circ} \mathrm{C}$, which is why minimum temperature thermometers have typically been replaced with alcohol thermometers. Record temperatures can be outside of the (calibration) range of some automatic weather sensors.

- A change in observer. The influence of the observer is especially notable for elements which involve some level of observer judgement, such as cloud or visibility, but also when the reading time is important. Some observers may not be following procedures over a long time 
(not measuring overflow of rain gauge, reading wrong side of minimum-maximum thermometer). In voluntary networks an observer change can also signal a relocation, which might not be documented elsewhere.

- A change in observation procedures. For instance, a change in observation time, which often happens simultaneously in the entire network and can also implicitly happen by regulatory changes in the local times. This is important for fixed hour measurements, but also for the daily minimum and maximum temperatures (Vincent et al., 2009; Degaetano, 2000; Vose et al., 2003; Karl et al., 1986) Changes in maintenance may be important, for example the painting, cleaning and replacement schedule of the screens. With AWS loss of ventilation due to icing may not be noticed and damage and soiling may be detected later. Changes in calibration procedures can potentially affect entire network.

- A change in the data processing. For example, in much of the world the daily mean temperature is computed from the daily maximum and minimum temperatures. However, early measurements in Europe were often made at fixed hours and the mean temperature computed from them, sometimes in combination with the daily minimum or maximum temperature. With AWS many different definitions of the daily mean have become possible. Historical Quality Control (QC) and validation procedures have changed, as well as the ability to do QC. For precipitation, how solid precipitation was measured and converted to the equivalent of liquid precipitation amount for archiving also matters notably (Wang et al., 2017).

- Digitisation and database errors. Digitisation may produce inhomogeneities, for example, when a minus sign is forgotten during digitisation of a section of data on paper (when the sign is indicated in colour). Data from stations with the same or similar names may be mixed up. In global databases the data can come from several different sources with unclear provenance. As a result stations with similar names may be mixed or slightly different series originating from the same station may be kept as different series. Also metadata, such as the station location and units, can be wrong. Typical database errors are values that are ten times too large, especially for precipitation.

The structure of this chapter is as follows. There are two main types of homogenisation: adjustments based on physical principles and adjustments based on statistical evidence. Section 3.2 explains the difference in more detail and explores how these two lines of work can strengthen each other. Parallel measurements, i.e. two or more measurement setups observing the same weather, are important for understanding and quantifying inhomogeneities (Section 3.3).

The next four sections explain the basics of statistical homogenisation. This is a two-stage process. The first stage is to detect potential inhomogeneities, which can utilise metadata, statistical methods and preferably a combination of the two (Section 3.4). The second stage is to adjust the data to account for the impact of those inhomogeneities (Section 3.5). Statistical homogenisation is mostly based on comparisons with neighboring reference stations; what makes a good reference is discussed in Section 3.6. How to select candidate stations and how to prepare the data for statistical homogenisation is the topic of Section 3.7.

A subsequent block of three sections treats statistical homogenisation topics that are important in specific cases. Section 3.8 is about network-wide inhomogeneities, which are important for largescale biases and more difficult to remove. Specific challenges for large multi-national datasets are the topic of Section 3.9. Some algorithms may require multiple rounds of homogenisation; Section 3.10 gives guidance on how to do that right. 
The ensuing two sections are important organisational issues: How to operationally maintain a homogenised dataset (3.11) and how to engage with network managers to avoid inhomogeneities and better assess their impact (3.12). The last section of this chapter (3.13) reviews the best practices for homogenisation.

\subsection{Physical and statistical homogenisation}

Whether a homogenisation method is called physical or statistical depends on how the size of the corrections are computed. Sometimes the main evidence we have that the candidate station has an inhomogeneity is statistical in nature: a large jump in one time series or a candidate station that behaves clearly differently than its neighbors. In such a case the corrections need to be computed statistically and we speak about statistical homogenisation. It is also statistical homogenisation when the date of the break is determined by metadata, but the metadata does not provide information on the size of the break.

Sometimes the reasons for the inhomogeneities are known and this understanding can be used to make more accurate adjustments. For example, when the time of observation will change, parallel observations made at both reading times can be used to correct for this problem (Vose et al., 2003; Vincent et al., 2009). When we use our knowledge about the reason of inhomogeneities for homogenisation we refer to this as physical homogenisation, even if the reason is not strictly physical (for instance, a factor ten missing for precipitation data, or mix ups of units).

When there is a documented station move, we know physically what happened, but the size of the jump needs to be determined statistically, thus this is still considered statistical homogenisation. The use of parallel data to estimate the size of the jump (for a relocation), is considered using a very good reference station and would be statistical homogenisation.

Physical homogenisation can also include statistics. For example, for the time of observation changes in the USA, NOAA developed a correction method based on hourly observations (Vose et al., 2003). To be able to correct stations, which only have daily observations, multiple linear regression was used to compute monthly time of observation bias corrections. Predictors were the station coordinates (time zone, latitude and longitude), observation hour, average diurnal temperature range and average day-to-day temperature difference (Karl et al., 1986). Similar methods were developed for Canada to account for a change in observation time from 0000 to 0600 UTC in 1961 (Vincent et al., 2009), whilst more recent 1-minute resolution data was used to assess the expected impact of an observation time change from 0000 to 0900 local time in 1964 at some Australian sites (Trewin, 2012).

When both physical and statistical methods are possible, it is recommended to compute both adjustments and compare them with each other. Comparing both types of adjustments can identify problems. Whether physical or statistical homogenisation is preferred for the actual corrections depends on the accuracy of the corrections. In most cases the physical adjustments are more accurate, but not always. First applying physical adjustments may make the remaining inhomogeneity too small to detect with statistical homogenisation, but still large enough to be climatologically problematic on the large-scale. Thus choosing the most accurate method may give better results than applying both methods after another.

It is the best to use a physical-based relationship to estimate the adjustments in cases where known physical relationships between variables are sufficiently robust that adjustments using these are likely to give better results than statistical homogenisation. For example, Wan et al. (2010) used a logarithmic wind profile to adjust surface wind speed data for anemometer height changes. Wan et 
al. (2007) used a hydrostatic model alone and in combination with a statistical model to detect and correct errors in both station and mean sea level pressure data due to errors in station elevation. ${ }^{2}$ However, physical-based adjustments need accurate metadata information (such as anemometer heights, station elevations) and may also need other data (such as dry-bulb temperature and surface roughness in the examples above). These metadata or data are often not available.

Physical homogenisation is mostly additional work because it can typically only be applied to a part of the inhomogeneities and statistical homogenisation should thus always also be applied to correct likely remaining ones. It is consequently mostly applied in situations where physical methods are highly accurate or even exact (e.g., digitization problems), and where statistical homogenisation is expected not to function well (e.g., low station density).

\subsection{Parallel measurements}

The best possible set of candidate and reference series is a parallel observation of the old and new situation (for example, a new instrument system is introduced, but the old system is continued for a period of some years after the new system commences).

Recommended practice for major changes in observations is to carry out a period of parallel observations between the two systems. WMO guidance for the optimal period of parallel observations is not fully consistent. CIMO (2014) suggests a minimum of 12 months for wind speed and direction, 24 months for temperature, humidity, sunshine and evaporation, and 60 months for precipitation (and also notes that "A useful compromise would be an overlap period of 24 months"), but WMO (2011) states that "at least one year, and preferably two or more years". This wide range of overlapping periods required reflects the fact that longer is better, but that making shorter parallel measurements should not be discouraged by specifying long periods because they are better than none at all.

These recommended practices are not universally followed (and adherence was less common historically than it is now), and parallel observations are an option that is not available where an inhomogeneity arises because of an unexpected change happening around the station (such as changes in the local site environment) rather than at the station itself. Adherence can be improved by making the management of changes part of the operational practise; see Appendix A for an example of the guidance on managing changes used by the Dutch weather service.

Parallel observations are most valuable if the "old" part of the parallel observation system is representative of conditions before the start of the parallel observations period. However, a common scenario is that the old site environment was changed during the period of parallel observations, which makes the parallel observations unrepresentative of the old site environment. Comparison with neighbor station data could help identify this problem and is highly recommended.

In case there are no parallel measurements available one can consider making parallel measurements with the equipment used before and after the break, or if the equipment is not available, with replicas created for the experiment. This is especially recommended to study the influence of historical transition that affected a large part or the entire network (Brunet et al, 2011; Mekis et al. 2011; Quayle et al., 1991).

\footnotetext{
${ }^{2} \mathrm{~A}$ hydrostatic model represents the relationship between station and sea level pressures, which is also dependent on dry-bulb temperatures.
} 


\subsection{Statistical detection/determination of change point \\ 3.4.1 Introduction}

Metadata should never be assumed to be complete and statistical determination of breakpoints should always be applied. For a homogeneity test, the null hypothesis is that the candidate series is homogeneous; and the alternative hypothesis is that the candidate series is not homogeneous (has one or more change points). In general, a statistical test works by comparing the value of a test statistic to its critical value corresponding to the chosen level of significance $\alpha$ (usually $5 \%$ ). The null hypothesis is rejected when the test statistic value exceeds the critical value. The significance level $\alpha$ is the chance for the null hypothesis to be rejected wrongly.

For a documented change point (i.e., the time of change is documented in metadata and known to the person analysing the data), one still needs to use a statistical test to determine whether or not the documented non-climatic change is statistically different from zero at a chosen significance level. In case an inhomogeneity is statistically significant it is possible to compute the adjustments sufficiently accurately.

Inhomogeneities are mostly abrupt, but can also be gradual (for example, in case of growing vegetation or urbanization). In statistical homogenisation we typically model inhomogeneities as a step function. This also works well for gradual inhomogeneities because there are often additional jumps during the gradual inhomogeneity. Furthermore, fitting linear trends to model gradual inhomogeneities is often not accurate as their behavior in time can be nonlinear. Thus in practise homogenizing gradual inhomogeneities with multiple breaks works well (Venema et al., 2012).

Most of the homogeneity tests are developed to detect mean shifts and thus cannot detect any variance-shift or probability distribution change that is not accompanied by a mean-shift. A variant of the Kolmogorov-Smirnov (K-S) test developed by Dai et al. (2011) can be used to detect unknown changes in the probability distribution (including variance-shift) of the data. Unfortunately, this method has not been included in any data homogenisation software. Szentimrey (2018) is working on a method for the homogenisation of mean and standard deviation for normally distributed data.

After a list of change points have been determined to be significant non-climatic change points, one need to estimate the adjustments that are needed to homogenize the candidate series; this is discussed in Section 3.5. Detection and correction is mostly performed by comparison with neighboring stations; more information on reference series can be found in Section 3.6. Readers are referred to the Methods Chapter 4 for detail descriptions of the software packages available for detecting/testing change points and estimating adjustments used in climate data homogenisation.

\subsubsection{Incorporating metadata in statistical tests}

A statistical break test provides an estimate of change point position. Its accuracy depends on the signal to noise ratio (SNR) defined as the variance of the break signal divided by the variance of the noise signal. If the SNR is well above one, the break position is very accurate.

Finding documentation for a break can be time consuming. The statistical evidence for a break can reduce the period for which the documentation needs to be investigated. The SNR gives guidance on how broad this period would need to be. For smaller SNRs errors of several data-points before or after the true change point are possible; see Figure 3.A. If the SNR is low enough a random segmentation of the time series can explain as much of the actual break signal as the segmentation estimated by a homogenisation method; see Figure 3.B. 
When combining statistically estimated breakpoint location with documented breaks it should be taken into account that also the metadata can be wrong. The likelihood of metadata being wrong and the uncertainty in the data is very situation dependent and subjective. Typically, if the metadata provides a specific date, metadata is more accurate, but if the statistical evidence is strong it can take precedence.

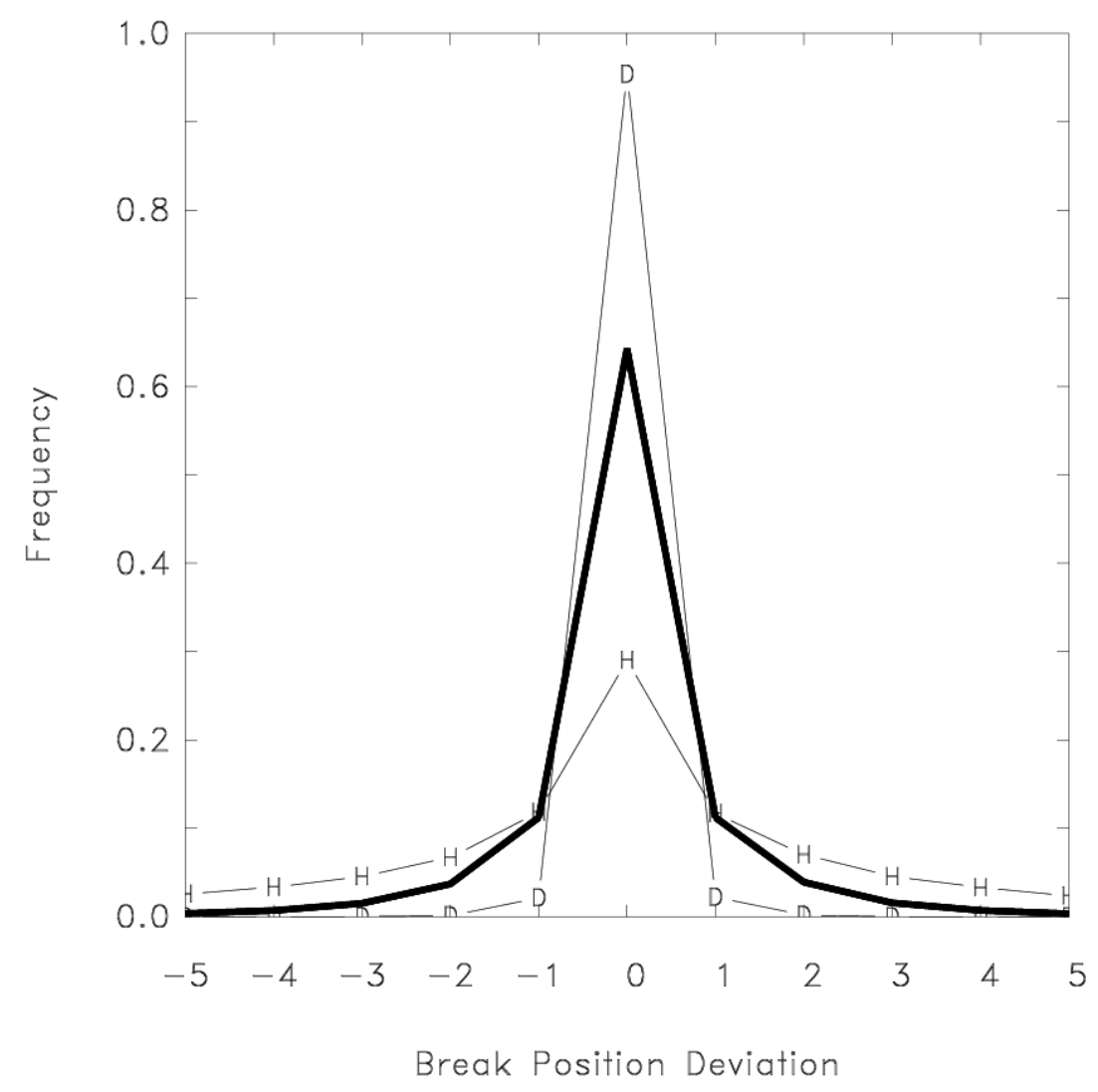

Figure 3.A. The distribution of the break position deviation for three different signal-to-noise ratios (SNR). The thick line is for a SNR of 1, i.e., for the case where the break variance is as large as the noise variance. The line marked with D's has the double SNR and the line marked with H's half the SNR. Figure from Lindau and Venema (2016). 


\section{Skill of standard search versus an arbitrary segmention}

7 breaks within 100 time steps, 1000 repetitions

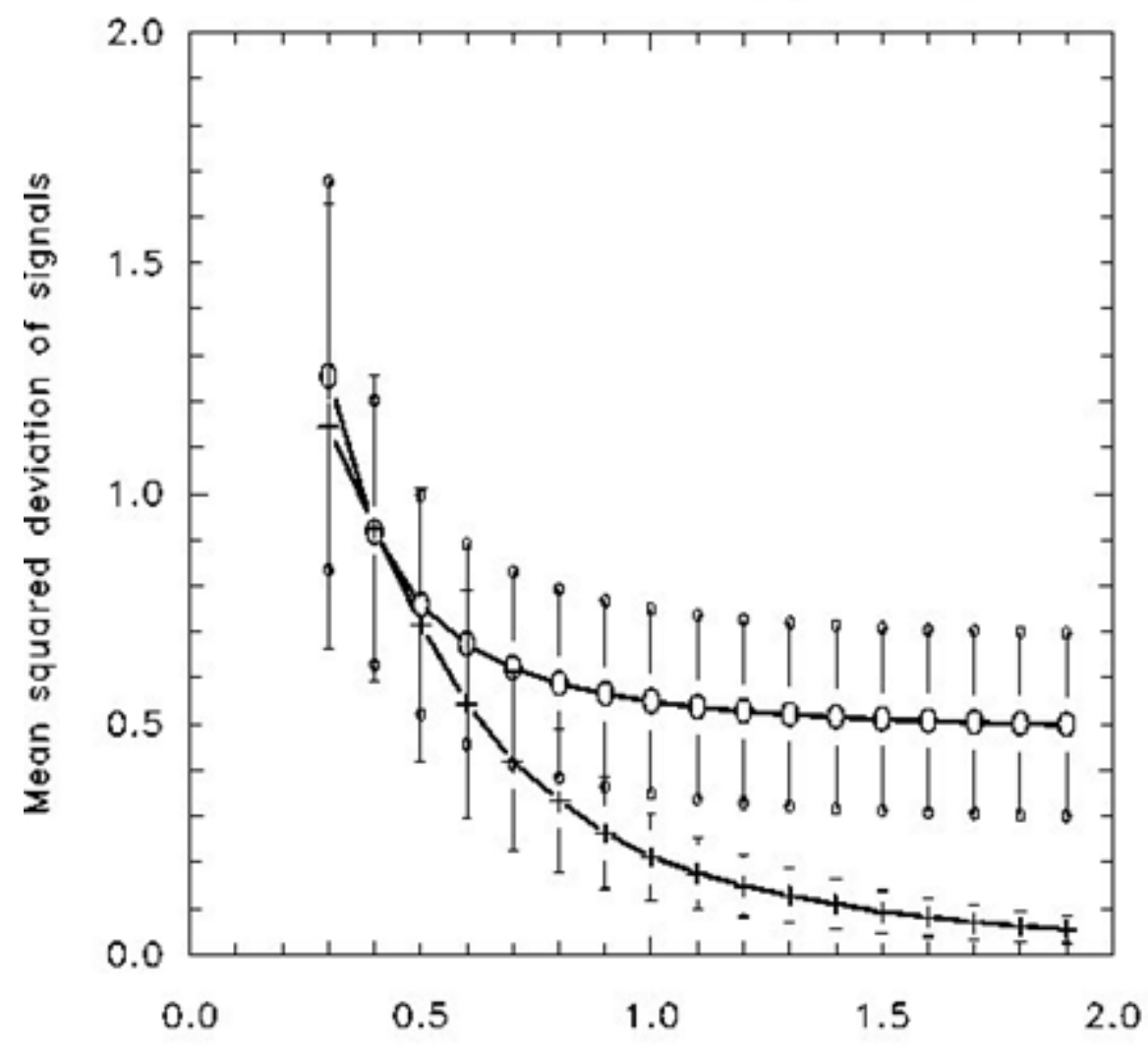

Signal-to-noise ratio SNR

Figure 3.B. The accuracy of the estimation of the break signal as a function of the signal to noise ratio (SNR). When the SNR is high the break signal is accurately estimated and the squared deviation between detected and inserted signal is small. A problem is that also a random segmentation can explain half of the variance of the break signal. When the SNR is about 0.5 the segmentation of the homogenisation method is just as good as a random segmentation. Because the detection breaks explain both noise and break variance, the variance is larger than expected in case of noise and the detection is statistically significant. The problem is that the algorithm is right to detect that the observations have inhomogeneities, but is unable to determine their positions. See Lindau and Venema (2018) for details.

The statistical tests for detecting unknown change points are different from those for determining the statistical significance of documented change points. For one documented breakpoint a t-test for the difference in the mean before and after the break can be used. In case of an unknown breakpoint many positions are tested (multiple testing problem) and the maximum expected difference under the null hypothesis is thus larger. The multiple breakpoints methods used in climatology have also been designed for unknown breakpoints. The commonly used tests for detecting single unknown change points (mean shifts) are maximal $t$ tests (e.g., standardized normal homogeneity test of Alexandersson 1998, the penalized maximal $t$ test of Wang et al. 2007) or maximal $F$ tests (e.g., the penalized maximal $F$ test of Wang 2008b, the two-phase regression model based tests of Lund and Reeves 2002, the multiple regression based test of Vincent 1998). 
The commonly used tests for determining the significance of documented change points are the regular Student $t$ test when a reference series is used and a regular $F$ test when the series is tested without using a reference series or when a trend difference between the candidate and reference series is suspected. The critical values for the regular $t$ or $F$ test are much smaller than those of the corresponding maximal $t$ or maximal $F$ test (see, for example, Lund and Reeves 2002; Wang 2003). Thus, the use of the regular $t$ or $F$ tests for detecting unknown change points would lead to too many false alarms (declare many change points that are actually insignificant).

When metadata is available, one can first use a statistical test to identify significant unknown change points; then, one can add in all documented change points that are not already in the list of Type-1 change points, to test their statistical significance. This is necessary because some documented changes in observing site or procedure or instrumentation might not induce any significant change in the candidate data series, while the statistically identified change points should be taken into account when testing the documented breakpoints using the homogeneous subperiod before and after the documented break.

To avoid the multiple testing problem the test for documented breakpoints should only be used for a limited number of breakpoints where the documented cause suggests a high likelihood of a break, such as relocations and screen-type changes. It is common to only use tests for unknown breakpoints.

\subsubsection{Options if no useful reference station exists}

There exist observational series that do not have any useful reference available, such as temperature observations in northern Canada (most of them are 400-800 km apart from each other), on remote islands or in the Antarctic. What matters for homogenisation is the signal to noise ratio; for wind, precipitation or humidity this will be low at much smaller distances, than for temperature and especially for sea level pressure. These stations are important because they have a large weight in regional or global mean time series because remote sites represent a large area and this happens more for scientifically interesting data from early periods. These situations are best solved with expert judgement and by retrieving and studying metadata. The following approaches can be used to homogenize data from such remote sites.

First, one can look for other related variables/data sources to use as reference. For example, for coastal and island stations, sea surface temperatures may be another reference series possibility (Cowtan et al., 2018). However, air and sea surface temperature do have different trends and variability. Cloud cover has been used to homogenize sunshine duration observation. Care must be taken that this does not remove variability in sunshine duration due to variations in cloud microphysics and aerosols. Surface air temperatures from a reanalysis dataset can be used as a reference to test surface air temperature recorded at a remote site, keeping in mind that reanalysis data have inhomogeneities of their own.

Second, there are homogenisation methods that can be used without using a reference series (absolute statistical homogenisation). However, homogeneity test without a reference series is much less reliable and should never be done with any automatic procedure. One should visually inspect the original and de-seasonalised candidate series, as well as study all available metadata to determine the final list of change points to be adjusted. The higher uncertainty of such homogenised datasets should be quantified and clearly communicated.

An important reason why absolute homogenisation is less accurate is that the series is more noisy. This noise is, furthermore, partially from long-term variations in the climate system, which is harder to distinguish from inhomogeneities than uncorrelated noise. The problems can be reduced by 
identifying and modelling the low-frequency variations within a homogenisation procedure, for example, using the method developed by Wen et al. (2011). An example of application of this method to the Fort Nelson (Canada) cloudiness time series is shown in Fig. 3.3: It identified correctly two shifts, along with a 12.5-year cycle, an annual cycle, and a negative trend; the combination of these components is shown as the blue curve in Fig. 3.3. If the 12.5-year cycle in this time series were ignored, i.e., if the PMFred algorithm were applied to this time series directly, it would identify three false change points and fail to identify the true change point. Such noise reduction techniques work especially well for absolute homogenisation, but can also be explored for relative homogenisation.

As mentioned above reference series may be derived from other variables. For example, Wan et al. (2010) and Minola et al. (2016) used geostrophic wind speeds derived from sea level pressure gradients over a triangle area (formed by three stations of surface pressure observations) as a reference series to homogenize surface wind speeds data from stations within the triangle area. Dai et al. (2011) used empirical relationships between the anomalies of air temperature and vapor pressure derived from recent observations when dewpoint depression (DPD) reports are available under these conditions to adjust artificial sampling effects by estimating missing DPD reports for cold $\left(\mathrm{T}<30^{\circ} \mathrm{C}\right)$ and dry (DPD artificially set to $30^{\circ} \mathrm{C}$ ) conditions. For coastal and island stations, sea surface temperatures may be another reference series possibility.

Reanalysis is independent of surface data for most variables and thus has potential as a reference series where no other suitable reference series exist. This approach has been used for the homogenisation of wind on the Iberian Peninsula (Azorin-Molina 2014). For upper air data, reanalysis data is used in the RAOBCORE and RICH datasets (Haimberger et al., 2012).

Because upper-air temperatures (on which reanalyses are based) generally have longer decorrelation length scales than surface temperatures, reanalyses may be of value as a reference series where no good surface neighbours exist. For the same reason it is expected that inhomogeneities in the reanalysis would be visible in the difference times series with many stations, which may allow the operator to determine whether the inhomogeneity is in the station data or the reanalysis.

Large-scale break inhomogeneities are present in reanalysis data when satellites datasets are introduced or change. Atmospheric models often have (regional) differences with observations. When more observations become available the reanalysis can thus (gradually) shift from the model base state to the observed climatology.

Homogenisation based on parallel data can be used in this situation when metadata on the observations is good. For example, Mekis et al. (2011) analysed data from a large number of parallel measurements with historically used rain gauges to homogenise the precipitation records in Canada.

If the data contains a clear break, absolute homogenisation can also be used to determine this date and to disregard the data before this cut-off date. In case of data sparse regions, such as the Arctic, not using such data can, however, also lead to biases. In case of data from early periods digitisation of more data may be able to resolve the conflict. 


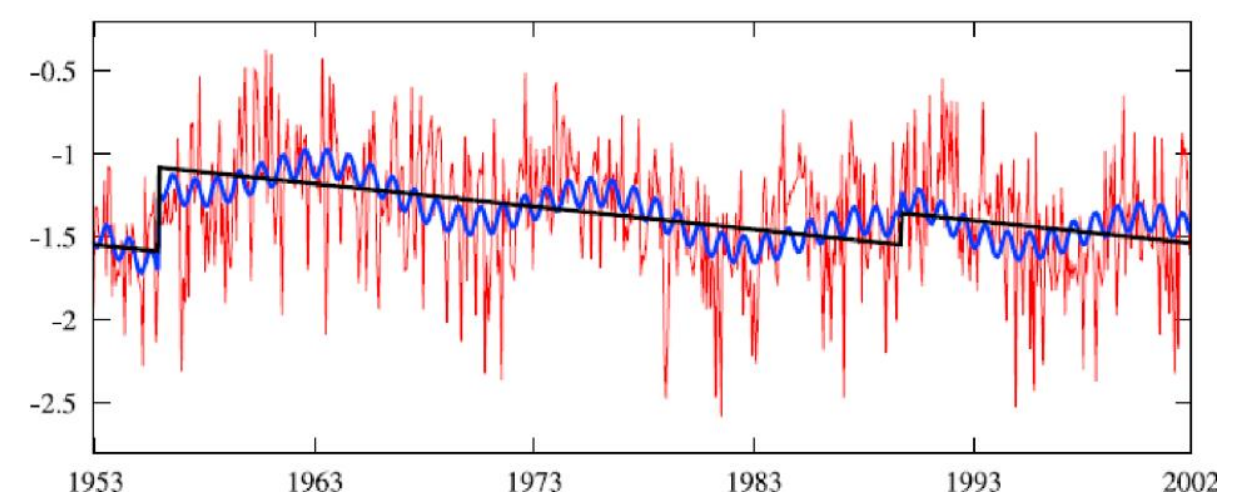

Figure 3.3. The Fort Nelson (Canada) cloudiness time series (red) and the climate signal and shifts (blue curve) identified by the method of Wen et al. (2011). The thick black line shows the trend and shifts, excluding the periodic components. This is part of Fig. 8 of Wen et al. (2011).

\subsection{Statistical adjustments}

The most accurate way to compute corrections is to consider all corrections simultaneously; this principle is called joint correction. Such a method was introduced by (Caussinus and Mestre, 2004), which decomposes the raw data into a regional climate signal for all stations, a step function for every station to model the breaks and noise for every station, which is minimised. This method was able to improve the corrections for nearly all contributions to the HOME benchmarking study (Venema et al., 2012) that did not use this method yet (Domonkos et al., 2011).

Alternative methods correct one break after another, which leads to an accumulation of errors in series with multiple breaks, especially in case of short homogeneous subperiods between breaks whose correction has a large uncertainty. This can be a reason not to set breaks close to each other; another option may be to treat two breaks close in time as a single break in correction, using data before the first break and after the second break as the basis for longer-term adjustments, and treat data between the two breaks separately. As an example, in the Australian ACORN-SAT methodology breaks are at least 4 years apart.

These correction methods can be applied to annual, seasonal and monthly data. Many inhomogeneities have a seasonal cycle. Numerical experiments with the PRODIGE homogenisation method on the HOME benchmark dataset showed that for temperature monthly corrections were most accurate. Because of the seasonal cycle of the inhomogeneities annual corrections performed less well. For precipitation annual corrections were most accurate, although the inhomogeneities did have a seasonal cycle. However, the uncertainty of the estimated monthly corrections was likely too large, leading to less accurate homogenised data. This is likely a good rule of the thumb, although it may be that in sparser networks than the typical European networks studied in HOME temperature corrections behave similarly as precipitation corrections.

Correction methods for the distribution of daily data can also be used to correct the seasonal cycle by applying them on data with the seasonal cycle, which typically dominates the total variance (Trewin and Trevitt 1996; Della-Marta and Wanner 2006; Mestre et al., 2011, Wang et al. 2010, Wang et al. 2013, Trewin 2013). For example, when a Canadian station near the St. Lawrence River was moved to an inland site, the inhomogeneity in the daily maximum surface air temperature series 
shows a clear seasonal cycle (see Fig. 3.4, right panel). The inland site is much warmer in summer, and a little colder in winter, than the near-river site.
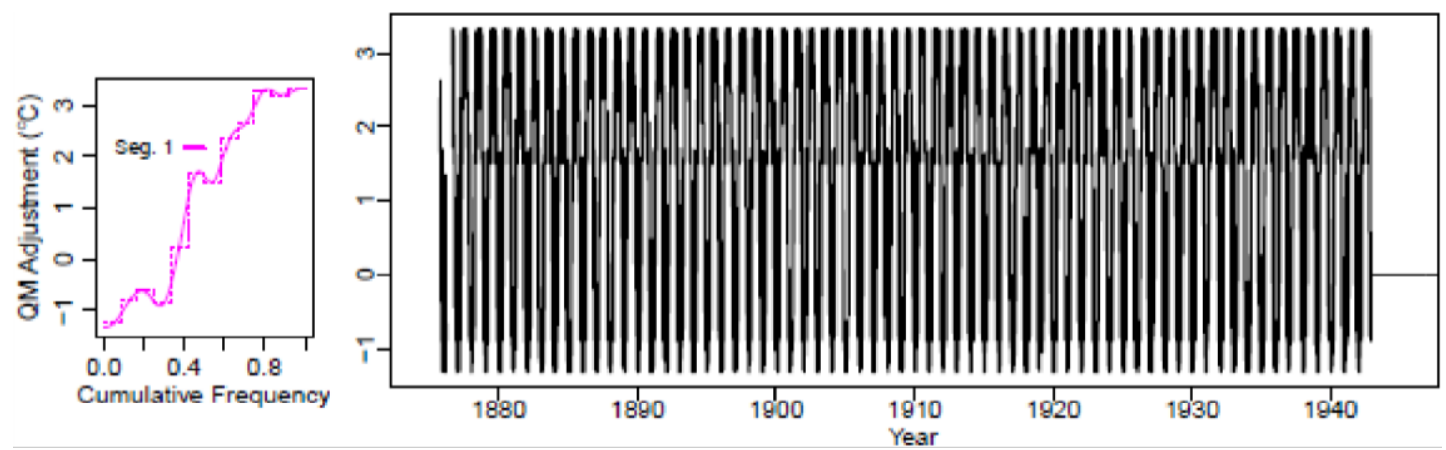

Figure. 3.4 Effect of relocating a station from a near-river site to an inland site on daily maximum surface air temperatures. Shown here is the distribution over quantiles (left panel) and time series (right panel) of the quantile matching adjustments estimated from a difference (candidate-minusreference) series, which are needed to adjust the near-river site data. This is part of Fig. 1 of Wang et al. (2013).

One practical issue is whether the whole homogeneous sub-period of data record should be used to estimate the adjustments. By default the full period is used to make full use of the limited amount of data to estimate the corrections, but sometimes it may make sense to deviate from this. In case the homogeneous subperiod is long, the added benefit of more data diminishes, while the risk of remaining inhomogeneities or noticeable differences in climate change between candidate and reference increases. This is especially the case when the reference is not optimal. When absolute homogenisation is applied it is common to limit the periods to 10 years before and after the break.

An additional issue, as discussed further in section 3.3 is where the period immediately before or after an inhomogeneity is not representative of the broader long-term behaviour of the station. This can occur, for example, where a station is moved after a sudden deterioration in its exposure; in such a case, it would be appropriate to exclude data between the change in exposure and the site move in making longer-term adjustments (see figure 3.5). In practice, issues of this type are often difficult to detect in statistical testing. Also in cases where the date of the break is uncertain, has a SNR below one (Lindau and Venema, 2016), or the statistical breaks and the metadata suggest different dates, it can be justified to exclude some data around the break when estimating corrections.

The use of composites without removing series with breaks is not recommended for computing corrections. The breaks that bias the network mean changes most are those that occur in all stations. When this happens over a short period, the reference would have a similar bias as the candidate and the large-scale bias would largely remain. 


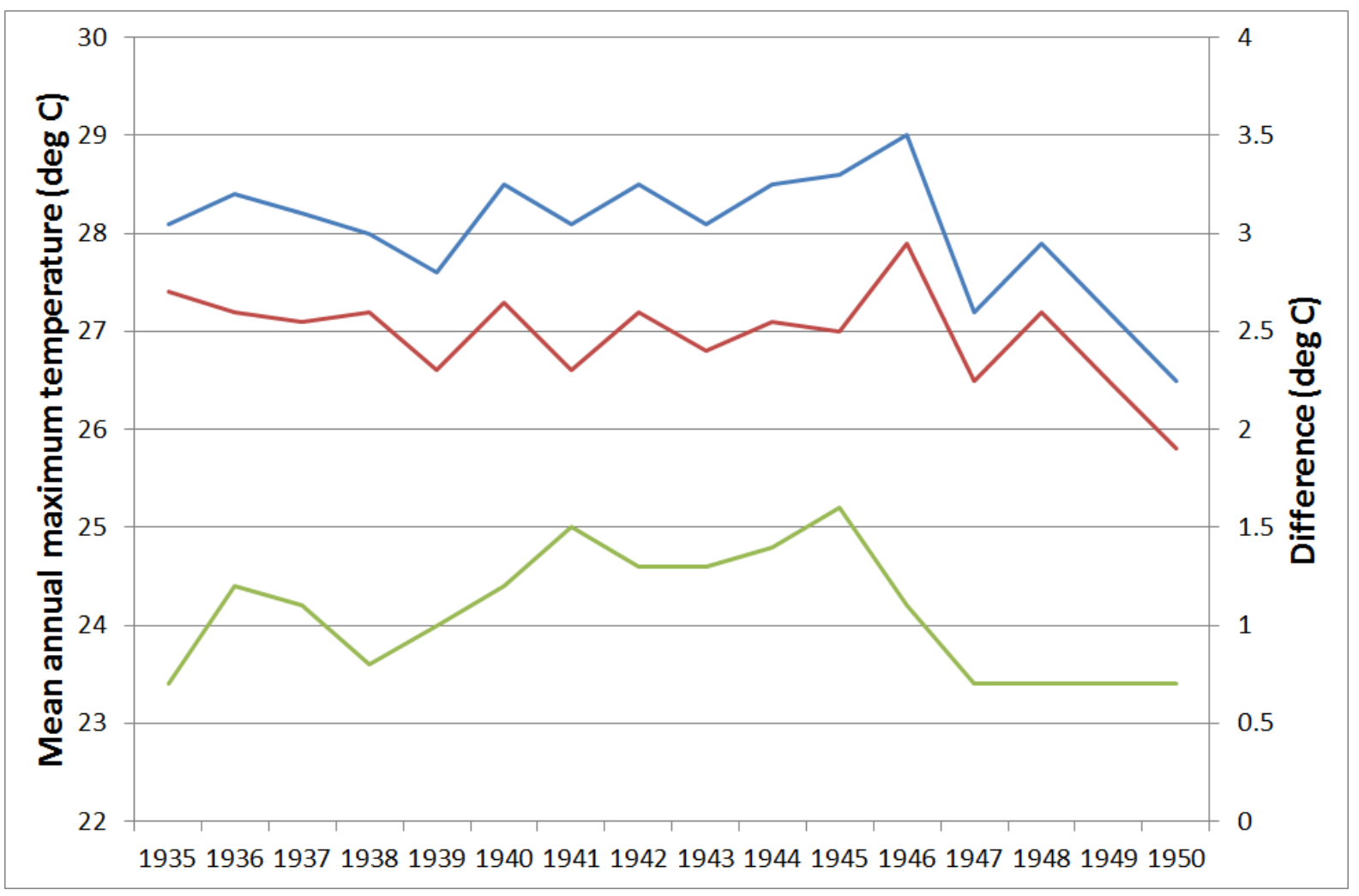

Figure 3.5. An example of unrepresentative data before a change. At Gayndah, Australia (blue line), the screen deteriorated progressively after 1940, before it was replaced in October 1945. The maximum temperature difference (green line) between Gayndah and the mean of three reference sites, Dalby, Brisbane and Emerald (red line), increased from $1.0^{\circ} \mathrm{C}$ to about $1.5^{\circ} \mathrm{C}$ in the years before the screen change, before dropping to $0.7^{\circ} \mathrm{C}$ after the change.

\subsection{Reference series}

The task of detecting, and adjusting for, an inhomogeneity in a climate time series is made more challenging by the fact that any climate time series will have substantial statistical noise in it, which arises from natural climate and weather variability and measurement errors. This can make inhomogeneities difficult to find - in the time series under consideration is, for example, a monthly temperature time series with a standard deviation of $1{ }^{\circ} \mathrm{C}$, then it will be difficult or impossible to detect a $0.4{ }^{\circ} \mathrm{C}$ inhomogeneity by statistical methods.

A common method of addressing this problem is comparing the candidate stations to neighboring reference stations. The most typical example is to compute a difference time series of the candidate and a reference and detect and adjust the series based on this difference series. What to do in case to reference stations are available is discussed in \$3.4.3.

Natural variability that exists in a candidate series will also exist in the reference series, and hence creating a series that compares the candidate and reference series will remove much of the influence of natural climate variability while retaining the effect of the inhomogeneity at the candidate site.

Another advantage of using a reference is that no assumptions need to be made on the statistical nature of the climatic variability as this is taken out. The difference time series can be assumed to contain white noise (or auto-correlated noise) and inhomogeneities. Typically, a reference series will comprise data from one or more locations near the candidate station. 
A reference data series can be a composite reference computed from several neighbor stations or a single neighbor station (pairwise homogenisation). In the latter case, inhomogeneities in the comparison series can belong to the candidate as well as the reference and multiple pairs have to be investigated to determine which station the break belongs to.

There are four considerations to select or weight reference series. 1) Together the references need to overlap over the full period of the candidate. 2) The weights should reduce the noise of the difference series, 3) reduce the influence of inhomogeneities in the reference and 4) enhance the similarity of the regional climate signal in the candidate and reference series.

These four considerations conflict with each other and the optimal solution is not clear. As a consequence many different methods are used to select reference stations and to assign weights to neighboring stations when computing a composite reference from them. Common weighting methods are weights based on correlations, the correlations of the first difference series, kriging (optimal interpolation) weights, the inverse distance from the candidate and height difference.

Stations are excluded when the distance or the height difference is too large or the correlation too low to select references with a similar climate.

In many cases, using distance is similar to correlations and the correlation between two stations will decay reasonably monotonically with the distance between them, but this may, for instance, not be the case in mountains where height is also important. The correlation matrix also may be anisotropic, for example, temperatures at an exposed coastal station are likely to be better-correlated with another similarly exposed station $100 \mathrm{~km}$ further along the coast than with a station $50 \mathrm{~km}$ inland.

In defining the correlation between two stations, we can let $B(t)$ and $R(t)$ denote the candidate series and the potential reference series, respectively. The series $\Delta_{B}(t)=[B(t)-B(t-1)]$ and $\Delta_{R}(t)=[R(t)-R(t-1)]$ are called the first difference series. The correlation between the first difference series $\Delta_{B}(t)$ and $\Delta_{R}(t)$ is often used to select reference series, because this correlation value will be much less affected by any inhomogeneities that might exist in the candidate and/or reference series (an inhomogeneity will only generate one bad value in the first difference series, but a segment of bad values in the candidate or reference series).

\subsubsection{Overlap}

In earlier periods data is typically sparser and it becomes harder to find well-correlated reference stations. To ensure having reference stations in the early period, also references with poorer correlations will have to be part of the set of reference stations.

The theoretical minimum number of stations needed for statistical homogenisation is 3 . In praxis, one needs about 5 stations (4 references) to obtain good results also when the situation becomes more complicated. This requirement typically determines the beginning year of a homogenised dataset.

Typically networks have less data in the beginning (and sometimes near the end). To have overlapping stations for the early period this normally means having to select stations with lower correlations. When adding shorter series to a composite reference, the beginning or ending can produce an inhomogeneity. Similar problems can occur for missing data periods. 
When selecting or weighting references based on their correlations, one should take into account that the computed correlations have considerable uncertainties, especially in case of short series; see Figure 3.B. Thus a high correlation of a short series may be a coincidence and a longer series with a lower correlation more reliable.

Short series of a fews years are also problematic because the detectability of a break depends on being able to detect a statistically significant difference in the mean value before and after a break. Thus next to the size of the inhomogeneity also the number of samples, i.e. the lengths of the homogeneous subperiods, is important.

\subsubsection{Noise reduction}

Kriging makes an optimal estimate of the observation at the candidate given the observations of the references. A composite reference computed as a weighted average of the references using Kriging weights will reduce the noise of the difference time series optimally. The kriging weights are computed using the cross-correlation matrix.

Because of the other 3 considerations and for ease of use, the correlations themselves or inverse distances are also often used to compute the weights for a composite reference series.

The detectability of an inhomogeneity is mainly a function of the signal-to-noise ratio (SNR). In case of one breakpoint the SNR is often defined as the ratio of the size of that inhomogeneity to the standard deviation of the time series under consideration. Reducing the amount of noise in a time series by using a reference will increase the likelihood of detecting an inhomogeneity of a given size. As shown in Fig. 3.B, for the case of one single change point in a data time series of length $\mathrm{N}=600$, the chance for reasonably accurate detection of inhomogeneities increases from around $53 \%$ when the ratio $\frac{\Delta}{S D}=0.5$ to around $91 \%$ (99\%) when $\frac{\Delta}{S D}=1.0(1.5)$; it is $\geq 99.99 \%$ when $\frac{\Delta}{S D} \geq 2.0$. A lower signal-to-noise ratio is also associated with a larger uncertainty range of detection power.

In case multiple breakpoints in one series are considered, the more realistic case, the SNR is commonly defined by the variance (or standard deviation) of the break signal divided by the variance of the noise signal.

If the SNR is below 1, the date of the breaks will be highly uncertain (Lindau and Venema, 2016). When the SNR is around 0.5 the breaks found are not much better than random breaks (Lindau and Venema,, 2018). In such a case, the break test will correctly detect that the series contains breaks, but would not provide reliable information on the position of the break. Trying to obtain a SNR above 1 is thus a high priority. Above such a SNR the other 3 considerations gain in importance.

A pair of stations in pairwise homogenisation will have two times as many breaks than a difference time series based on a composite reference because the composite offers a better estimate of the regional climate signal than a single other station. Thus if the SNR is low, the use of composite references may be preferred. 


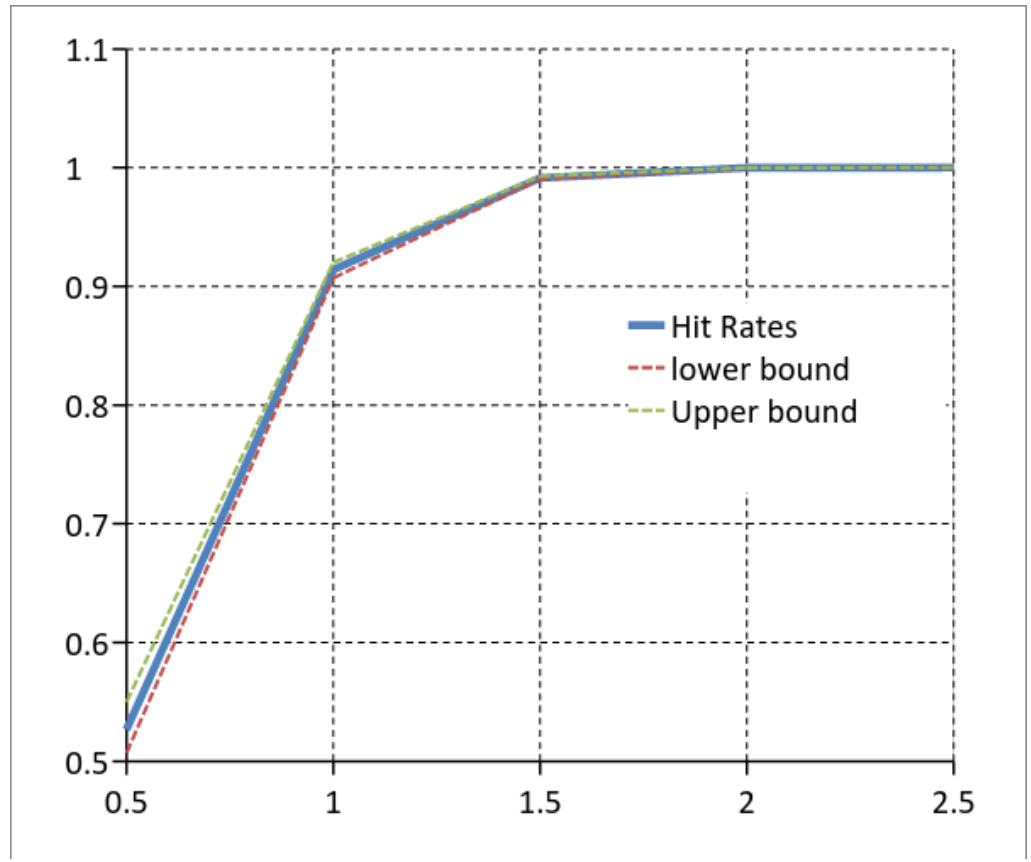

Figure 3.B Power of detection, i.e., hit rates (vertical axis) as a function of the ratio $\frac{\Delta}{S D}$ (horizontal axis) of the size $\Delta$ of an inhomogeneity to the standard deviation (SD) of the candidate series. This is based on the data in Table 1e of Wang (2008a); it is for the case of one change point in a series of length $N=600$. The lower and upper bounds represent the $95 \%$ confidence interval (uncertainty range) of the hit rates.

\subsubsection{Inhomogeneities in the reference}

A reference series should be homogeneous or at least be homogeneous in a sub-period in which the candidate series is inhomogeneous. Inhomogeneities in reference series can easily be mistaken as inhomogeneities in the candidate series. Such mistakes can be greatly reduced by visualization of the difference series or the regression fits. To reduce the risk that a composite reference has significant inhomogeneities of its own around the time of interest a sufficient number of reference stations needs to have significant weights.

In case of widespread inhomogeneities occurring in many or all stations over a short period one needs to be extra careful computing a composite reference. The composite may not contain visible jumps, but if this widespread inhomogeneity has a bias this will also be present in the composite reference. This is especially problematic for computing corrections.

Removing reference stations with breaks from the computation of the composite reference reduces this problem. Also carefully designed iterative procedures that correct the breaks in the reference stations may do so. Pairwise homogenisation methods are best suited for dealing with such difficult cases.

\subsubsection{Similar climate signal}

The selection of references with a similar climate signal is best performed by an expert based on an understanding of the local climate. In automatic methods, similarity is often estimated by the crosscorrelations, together with thresholds for the maximum distance and height difference and minimum correlation. 
However, a high correlation does not guarantee that the references have the same regional climate signal. Further objective criteria could be the Köppen climate classes, the size of seasonal cycle, daily cycle, exposure, soil moisture and vegetation.

In case of sparse networks, the number of reference stations may need to be limited to ensure they all belong to the same climate region.

All stations having a similar climate signal is especially important in detection methods with a step counting how many breaks were detected. This is sometimes used to collate the results of several break detection tests or in the attribution step of the Pairwise homogenisation Method where the station with the break is determined by the number of pairs that have a break. In case not all pairs belong to the same regional climate, it is possible that many far away reference stations falsely suggest a break, while it is ignored that a smaller number of the stations in the same climate region rightly do not. In such cases it is appropriate to select less reference stations or to apply higher weights to nearby stations.

\subsubsection{Using references}

References are mostly used in two ways, for normally distributed variables the difference between candidate and reference is used, for approximately log-normally distributed variables the ratio of candidate and reference. In the difference series approach, the test is applied to, and the adjustments are estimated from, the difference series $D(t)=[X(t)-Y(t)]$. The ratio series would be computed as $\mathrm{R}(t)=[X(t) / Y(t)]$. Temperature and most other variables stem from additive processes and have an approximately normal distribution. Precipitation and wind speed are multiplicative processes, which produce approximately a log-normal distribution. In some climates even the monthly averages of these variables may contain zeros. In this case one can transform the data to an approximately normal distribution and use a difference time series.

Less commonly the covariate approach is used where the reference series is used as a covariate in a regression based test (e.g., the multiple regression based test of Vincent 1998), in which case the test is applied to the residual series of the regression of the candidate series on the reference series, e.g., residuals $\varepsilon(t)=[B(t)-\hat{B}(t)]$ of the fitted regression $\hat{B}(t)=\hat{a}+\hat{b} R(t)$.

\subsection{Selecting the data to be homogenised}

\subsubsection{Which stations should be selected?}

There are two broad approaches which can be used for selecting the data to be homogenised in a national or regional dataset. (Sometimes, a homogenisation project will be directed at homogenizing a single station, in which case no further station selection is required after the initial choice).

The first is to include all stations which meet set criteria for factors such as length of record and data completeness, while the second is to only consider a selection of stations, chosen on criteria such as records of the best quality (e.g., best site standards, fewest documented moves) or geographic representativeness.

Both approaches have been used for major national datasets; for example, the United States national homogenised dataset (Menne et al., 2009) includes all available stations with a sufficiently long record, whereas the Australian national dataset (Begert et al., 2005; Brunet et al., 2006; Trewin, 2013) selects the 112 stations assessed to be of the best quality and representativeness out of a broader national network which is several times larger. 
A strong selection reduces the amount of work, which means that more attention can be given to single stations. Care should be taken that the SNR is above one. More stations have as advantage that regional climates can also be studied and maps can be produced.

\subsubsection{How to prepare data for homogenisation?}

Once a set of stations has been selected for homogenisation, the next step is to determine which data from those stations will be used in the detection process. Decisions made here are important because some inhomogeneities may have a seasonal cycle - for example, a change in the wind shielding of a precipitation gauge at a cold-climate site may have little impact on summer precipitation, but a large impact on precipitation in winter when much of it falls in the form of snow.

Three possible options are:

(a) To use only annual data (annual sum or mean, sometimes combined with magnitude of seasonal cycle).

(b) To use monthly or seasonal time series in parallel with each other (and/or with annual data).

(c) To use monthly or seasonal data (either in its original form or as anomalies) as a single time series (also referred to as serial or consecutive monthly or seasonal time series).

The use of annual data typically has the most favourable signal-to-noise ratio for inhomogeneities which affect all, or a substantial part, of the year. Compared to serial monthly/daily data, the lower SNR is, however, exactly compensated by the larger number of values (in case of white noise and as long as the breaks are on the 1st of January; Lindau and Venema, 2018). On the other hand, using annual data may result in missing inhomogeneities that only affect part of the year, or inhomogeneities which have opposite impacts in different seasons and cancel out in an annual mean (for example, where a site moves from an exposed coastal location to one further inland, it is likely to be warmer in summer but cooler in winter). Also using the seasonal cycle or method (b) can thus improve the results.

Using monthly or seasonal time series in parallel (that is, carrying out tests separately on time series for each month/season with one data point per year) allows the detection of seasonally-varying inhomogeneities. In some cases, a station which shows no significant inhomogeneity at an annual timescale may show significant seasonal signals, e.g., where opposite signals in summer and winter cancel each other out in an annual mean. This method requires the consolidation of information from the various monthly/seasonal tests with each other, and with annual values, to determine a final set of potential inhomogeneities. However, the length of these series is basically the same as the annual data series, which is much shorter than the consecutive monthly or seasonal series, while they are more noisy; this will reduce the detection power of statistical tests.

Some methods use monthly or seasonal data as a single time series, also called serial monthly or consecutive monthly series. This allows greater time resolution in detecting inhomogeneities and like parallel monthly avoids treating the year with the break as member of one of the homogeneous subperiods, but may also miss a seasonally variable signal as it will repeat each year. Such methods may also be complicated by the fact that, in some climates, not only does the mean value of a time series vary seasonally, but its variability may also have a seasonal cycle (for example, in most midand high-latitude locations in the northern hemisphere, temperature variability is substantially greater in winter than it is in summer). Also, autocorrelation in consecutive monthly or seasonal series is usually higher than in annual series and hence non-negligible and should be taken into account in the statistical test applied. 
Methods using annual data cannot determine the timing of an inhomogeneity, without reference to metadata, to a precision greater than one year. However, if the SNR of monthly or seasonal data is not large enough the break date will have a clear uncertainty (Lindau and Venema, 2016). Hence, the greater apparent precision which might be obtained from a monthly method can be partly illusory. The greater temporal precision may also sometimes be helpful in focussing the search for metadata.

Most widely used methods are types (a) for automatic methods and (b) for manual homogenisation. Methods of type (b) are likely to detect a broader range of inhomogeneities if applied properly.

\subsection{Network-wide issues and options for dealing with them}

On occasions, changes will occur that affect all stations in a national network, or a substantial proportion of those stations, at the same time or over a period of years. Examples of such changes include:

- A change in observation time - either explicit (such as the change in the observing period for daily data from 0000-0000 UTC to 0600-0600 UTC in Canada in 1961)) or implicit (e.g., Australian stations continued to observe at the same local clock time when daylight saving time was introduced in the early 1970s, introducing an effective one-hour shift in standard observation time during summer).

- A major change in instrument type - such as a change in standard thermometer screens (e.g. to AWS), or the introduction of a new type of radiosonde. (Upper-air observations are particularly susceptible to this type of change, as they do not involve much fixed infrastructure and hence it is possible for any change to be implemented quite quickly).

- A change in observation procedures or definitions - such as changing the unit of cloud amount measurement from $1 / 10$ to $1 / 8$, or a change of units (e.g., from Fahrenheit to Celsius).

- A change in algorithms used for data analysis - such as a change in the definition of daily mean temperature (e.g., from the mean of eight 3-hourly observations to the mean of the daily maximum and minimum).

Network-wide changes can be particularly challenging to deal with in a homogenisation process. Since they normally apply to most or all stations in a particular region, the use of reference stations from the same network, in the normal sense, will be of limited use both in detecting the inhomogeneity and in determining its likely impact. In addition, a change whose impact may not be significant, or detectable, at an individual station (e.g., a $0.2^{\circ} \mathrm{C}$ temperature inhomogeneity) may be significant in a national mean if it affects all stations in a network or in the global mean if it represents a technological or organisation change that was common.

Some potential strategies for addressing such changes include:

- Changes affecting the entire network are not always mentioned in metadata databases, which typically like changes to individual stations. Because they are important national events, they are often mentioned in annual reports.

- If a change affects most, but not all, of the stations in a network (e.g., it affects all automatic stations, but not manual ones), compare the affected and unaffected stations.

- Compare with observations near the border in neighboring countries that are unaffected by the change. (This is only effective where there are such stations, which normally requires a land border, and also requires access to observations from other countries, which may not always be easy to obtain).

- Compare with another data type which was not affected by the change. Examples include comparing temperatures at the surface with radiosonde temperatures at $850 \mathrm{hPa}$ (or 
reanalysis fields based on those), or comparing measured wind speeds with geostrophic winds derived from mean sea-level pressure fields.

- Use alternative data to indicate the possible impact of the change. For example, for a historical change in observation time, even though high-resolution sub-daily data may not be available from the period when the change took place, it may be possible to use highresolution data from recent years to estimate what the impact of a past observation time change may have been. For example, Vincent et al. (2009 and 2012) used hourly temperature data to correct biases in daily minimum temperatures caused by a change in observing time across Canada. In Austria (Böhm et al., 2010) and Spain (Brunet et al., 2006) parallel experiments were performed to quantify the changes due to the transition to Stevenson screens.

Such methods can be reasonably coarse in their results, and it may only be possible to quantify the impact of such inhomogeneities at a national or regional level (or to determine that a change had no significant impact at that level), without fully accounting for different impacts that any inhomogeneity may have had at individual stations.

Network-wide inhomogeneities can be solved in different ways depending on the resources available. The first version of the US Historical Climate Network dataset contained a physical adjustment for the transition from Stevenson screens (Cotton region shelters) to (MMTS) automatic weather stations. This transition happened in many stations over a short period of time and was consequently often difficult to homogenize using statistical homogenisation comparing a candidate station with a composite reference (the average signal over several neighbors), because these neighbors were often also affected. The adjustments were based on estimates of those candidates that had neighboring stations without this transition. After designing a new homogenisation method, making pairwise comparisons, it became possible to homogenize this difficult situation and the physical adjustments were no longer applied.

\subsection{Specific challenges for multi-national datasets}

Combining data from different networks and countries has its advantages as it reduces problem with network-wide inhomogeneities. However, developing a homogeneous dataset at a global or regional (multi-national) scale also has some particular challenges. These include:

- Large dataset size. Such datasets will often contain information from hundreds or thousands of stations. These will normally be beyond the practical size limit for manual homogenisation methods, requiring the use of automated methods.

- Limited access to metadata. As metadata is normally archived at the national level, with only the most basic metadata exchanged internationally, there is often limited or no capacity to incorporate metadata into the homogenisation process for multi-national datasets. (Even if metadata can be accessed, using it will often require interpretation of documents in the local language). Sometimes even determining exactly which station data come from can be challenging, with a major task in global data set development being identifying and consolidating duplicate data sets from different sources (e.g. Rennie et al., 2014). The sharing of metadata will hopefully improve with WMO OSCAR.

- Limited access to potential reference series and other relevant data. Often, in a multinational dataset only selected climate stations are shared for climatology, sometimes in addition other data is available, which are routinely internationally exchanged. In contrast, in national datasets, it is often possible to draw on additional data (e.g., shorter-term stations, which have too little data to be considered for a long-term dataset, but can still be useful as reference stations in specific sub-periods), as well as data from extra elements and sub-daily data, while global datasets are typically single-element datasets; ECMWF is working on a 
multi-element database where observations from one station are kept together (Dunn and Thorne, 2017).

These factors combine to make automated methods the most likely option for such datasets, and make it more difficult to find well-correlated reference series, making it more difficult to detect smaller inhomogeneities and increasing the uncertainty in those adjustments which are made.

One option is to draw upon national-level information to the extent which it is available. In the HadCRUT global temperature dataset, maintained by the UK Meteorological Office and the University of East Anglia, national-level homogenised datasets are used where they are available (Jones et al., 2012).

\subsection{Multiple rounds of homogenisation}

In case of manual homogenisation with methods using a composite reference it is normal for an initial homogenisation process to fail to fully address all homogeneity issues with the dataset under consideration. There are a number of reasons why this can occur; common ones include:

- Undetected inhomogeneities in one or more reference series, or in one of the stations within a parallel observations pair during the period of parallel observations.

- Anomalous climatic conditions around the time of an inhomogeneity resulting in an unrepresentative adjustment (for example, a particularly wet or dry period immediately before or after an inhomogeneity).

- Conditions at a site shortly before or after an adjustment which are unrepresentative of the longer-term record. One common scenario here is where a site move takes place because of recent building work near the old site; data from the old site after the building work started may not be representative of the old site prior to the building work, and therefore should not be used in determining the required adjustment for a long-term dataset.

The most effective way of dealing with these issues is to carry out a second-round homogenisation process. This involves carrying out homogeneity tests on the "homogenised" data - for example, by comparing with other homogenised stations in the region, or testing for anomalous trends at individual homogenised stations (i.e., check the spatial consistency of trends). Visual inspection of time series from the first round of homogenisation can also be useful in some situations.

Where issues are identified through the second-round process, depending on the nature of the issue, options for addressing them include:

- Repeating the homogenisation using a different set of reference stations (if possible). A useful approach, if a number of reference series are being used, is to estimate the size of the adjustment which would occur by using a single reference station, for each of the reference stations individually, and remove any reference station which generates results which are excessively anomalous relative to other reference stations.

- Using a different time period as the basis for adjustment. For example, if a station moved in 1951 but a new building was built near the old station in 1949, the period ending in 1948 (rather than the period ending in 1950 or 1951) should be used as the basis for long-term adjustment.

If one makes multiple rounds of homogenisation one should never assume that the data of a previous round was homogeneous and compute all corrections anew. Otherwise the solution may drift away from the truth by repeated homogenisation. 
Sometimes, even after a second (or third) round of homogenisation, some stations will still show anomalous trends relative to other stations. These may occur because of changes which gradually affect the local climate over an extended period (for example, being encroached upon by an expanding urban area, or increasing levels of irrigated agriculture in a district), or because of natural local effects (e.g., a coastal site being cooled by increased levels of coastal upwelling in the nearby ocean). If it can be established with a reasonable level of confidence that such anomalous trends have a specific non-climatic cause (such as urbanisation), one option is to remove them from the dataset, or to exclude them from some products based on the dataset (for example, not including stations influenced by urbanisation in assessments of long-term climate change).

\subsection{Operational maintenance of a homogenised dataset}

The initial development of a homogenised dataset is a substantial undertaking. Normally, a major purpose of homogenised datasets will be as the underlying dataset for products (for example, a national or global temperature anomaly), which means that for those products to continue to be updated, the underlying dataset needs to continue to be updated too. The advantage of automatic homogenisation methods is that they can easily be applied when new data comes in.

It is recommended that homogenised datasets be constructed in such a way that the most recent data are unadjusted, with older data adjusted (where required) to be homogeneous with the more recent data. This allows the dataset to be updated by appending new data without further adjustment, as long as those data remain homogeneous. An exception to this may occur where the older data are considered to be a more reliable long-term reference; for example, in a precipitation network, if the bulk of the network is manual but a small number of automatic stations are being introduced, it may be more appropriate to adjust the automatic stations to be equivalent with the earlier manual data for better spatial consistency of the network as a whole.

Over time, a homogenised dataset will start to become out of date. There are two major factors which contribute to this. Firstly, some stations which are part of the original dataset will close over time (sometimes replaced in the network by new stations nearby, which can be used as the basis for a composite, sometimes not). Secondary, new inhomogeneities may occur at stations which remain in the dataset.

It is recommended that a reassessment be undertaken of any homogenised dataset at least every 5 years. This reassessment should include:

- A check of the status of all stations in the existing dataset, and if they are closed or no longer reporting reliably, whether they can be replaced with an alternative station that can become part of a composite record.

- A search of recent metadata (covering the period since the last update) for all stations in the dataset.

- Incorporation of any relevant historical data that has become available (for example, data recently digitised as part of data rescue activities).

- In case of manual methods at least a statistical testing for inhomogeneities in the most recent part of the record, including a reassessment of the last few years of the previous version of the dataset, as inhomogeneities in the last (or first) few years of a time series are difficult to detect and quantify and the additional new observations may allow more reliable assessments to be made. Especially with automatic methods a full homogenisation of the full dataset is recommended. 


\subsection{Engagement with network managers}

The task of maintaining a homogenised data set is made simpler if there are fewer inhomogeneities, which need to be considered. While nothing can be done to prevent inhomogeneities which have already occurred, there is considerable value in managing a network in such a way as to reduce the number of inhomogeneities in current and future data, and/or to facilitate accurate quantification of any inhomogeneities which may occur. This is something which requires engagement between climatologists and network managers. This can be challenging, especially if different organizations are responsible for managing the observation network and for climate data.

Practices which can help achieve the goal of minimizing ongoing inhomogeneities include:

- Ensuring that proper field trials are carried out for any new observation system before they are implemented in the full network. These field trials should preferably be carried out for a minimum of two years (a single year can produce misleading results if that year is climatically unusual), and especially in large countries should take place in a range of climates representative of the full range of climates found within that country.

- Ensuring that parallel observations take place where any significant change occurs, that these start early enough to provide at least two years of overlapping data and are archived and shared.

- Identifying sites which are at risk of closure, or significant changes in their environment (e.g., through planned building works nearby), at an early stage, to maximise the time available for parallel observations with any new site.

- Where new sites are being chosen, selecting sites that match the topography and local environment of the old site as closely as possible, to lessen the risk that a climatically significant change will occur as a result of the site move.

- If possible select sites where the surrounding is unlikely to change in the coming decade to centuries.

Also important are the ongoing maintenance of metadata, and the appropriate use of station identifiers. In particular, station identifiers should not be re-used, as this creates a high potential for confusion and may lead to data being merged from two completely unrelated stations. The re-use of station identifiers is a particular problem for international datasets which depend on WMO station numbers, as under current systems (in the process of being updated), the supply of WMO station numbers is limited - the numbers have only five digits, and the first two are used as a country code.

Appendix A contains an example protocol for the management of changes in climate networks to avoid unnecessary changes and handle the unavoidable ones. This includes studying inhomogeneities (in advance) and storing the parallel measurements, which are made as experimental data in the central database.

\subsection{Best practices for homogenisation}

This chapter outlined a range of issues and considerations in the development of long-term homogeneous datasets. The extent to which these can be implemented will vary widely, depending on the access of the dataset developers to relevant data and metadata, the tools and computer systems available to them, the density of the underlying observation network, and the size of the dataset under consideration.

There are a number of principles which can be considered as best practice for data homogenisation, including the following:

1. Data homogenisation is most effectively done through a combination of statistical methods and metadata. If this is not possible (e.g., because metadata are not available, or because a lack of reference series makes statistical homogenisation difficult), homogenisation is likely 
to be less effective. Statistical homogenisation should always be applied; it cannot be assumed that metadata is perfect.

2. If there are known issues with a dataset (for example, a network-wide change of observation time), these should be dealt with before more station-specific inhomogeneities are considered. The use of reference series directly or in pair-wise comparison usually will not identify network-wide changes.

3. Reference series should be used in statistical homogenisation methods if at all possible.

4. It is important to try to get the signal to noise ratio above one.

5. Once a draft version of a homogeneous data set has been prepared, methods that assume the composite reference to be homogeneous need a second round of homogenisation, as discussed in section 3.11 above.

6. homogenised data sets should be fully updated at least every five years.

7. Where major changes are anticipated, parallel observations should be established and maintained for at least two years. An example document on managing network changes can be found in Appendix A.

8. When an adjusted dataset is produced, the adjustments should be properly documented and published. Such documentation should include the dates covered by the adjustment, whether the inhomogeneity was identified through statistical methods or metadata (and, if the latter, what the likely cause of the adjustment was). Summary statistics of the influence of adjustments (all and per metadata category) can help the user assess the quality of the data. Best practise is to share both the raw and homogeneous data as well as the metadata on the identified breaks.

9. The methods used in the development of any homogenised dataset should be properly documented in an accessible form. This should include a paper published in the peerreviewed scientific literature, but at a minimum should include a detailed description of methods used, which is available in the same location as the homogenised data themselves. Best practise is to write clear well-documented code taking into account that it will be published alongside the data.

While no method can guarantee a fully homogeneous dataset, and any homogenised dataset will have some level of uncertainty associated with the adjustments involved in creating it, following these principles should maximise the likelihood that a dataset will be sufficiently homogeneous that it can be effectively used for the development of long-term climate data products. 


\section{CHAPTER 4}

\section{Selecting statistical homogenisation software}

This chapter aims to guide the reader to numerical methods and software packages that can be used for various homogenisation tasks. There are multiple statistical homogenisation software packages, as described in Section 4.1. We will describe how we know about their performance (Section 4.2) and what the performance and abilities of the publicly available homogenisation packages are (Section 4.3). The considerations for selecting an appropriate package for the task at hand are illustrated in Section 4.4 by discussion various typical use cases. It is assumed that the reader has a basic understanding of the principles of homogenisation from reading Chapter 3.

\subsection{Statistical homogenisation packages}

The tables and descriptions below list the publicly available homogenisation packages that are used in climatology and their characteristics. New methods are regularly being developed and the list below should not be considered to be definitive; other methods also exist in the scientific literature but are not included in the list/table below as their software has not yet been released in a form which is usable by the broader community. These tables are kept up to date on the homepage of the TT-HOM: http://www.climatol.eu/tt-hom/.

Many of these methods were included in a benchmarking study as part of the HOME project (Venema et al., 2012). Section 4.2.2 contains further discussion of this study.

ACMANT is a homogenisation package for temperature and precipitation data. It is one of the most accurate automatic method we currently have to homogenize temperature networks without metadata.

AnClim implements all common detection and correction methods with one graphical user interface. The AnClim contribution to the HOME benchmark using an ensemble approach using many methods and settings had an average performance. But the package offers access to many methods, which can also be used by themselves in the more standard way, and together with ProClimDB (not free) helps automatize many tasks and works with databases.

Bayesian MDL is a multiple-breakpoint method that is freely available, but in a research stage and mentioned for completeness and developers of homogenisation methods (Li et al., ArXiv 2017).

Berkeley Earth is a homogenisation and interpolation method used for global temperature datasets. The homogenisation corrections are computed in the interpolation part. It thus also does not return station data, but a field or an estimate of the regional climate at the location of the station.

Climatol has moved to SNHT detection after the HOME benchmark, which has improved its performance. It automatically computes a full series for every homogeneous subperiod in the original data, and can make use of short series and available metadata.

GAHMDI solves the multiple-breakpoint problem with a global search algorithm (genetic algorithm). It is packaged together with HOMAD, a method to correct the distribution of daily data. No intercomparison and documentation beyond the two published articles is available. 
GSIMCLI uses geostatistical methods to compute the null hypothesis numerically using a Monte Carlo approach. It is a new method and there is no information from intercomparison studies on its performance. It provides a graphical user interface and automation for networks.

HOMER was designed as part of the COST Action HOME, but its performance was not benchmarked. It implements several multiple-breakpoint methods. Using the pairwise option, it is is the successor of the multiple breakpoint method PRODIGE and expected to be one of the best manual methods. It includes the ANOVA correction method, which is likely the most accurate correction method (Domonkos et al., 2013) The joint detection option (cghseg) is best not used alone.

iCraddock implements the Craddock test in a pairwise fashion. This manual method is subjective, but performs well for small networks.

MASH is an automatic homogenisation algorithm based on hypothesis testing that is designed to work with inhomogeneous references and uses a multiple breakpoint approach. It has an excellent performance.

ReDistribution Test is a single breakpoint test for vector wind.

RHTests implements several break detection tests taking into account auto-correlations and the distance from the edges. It is the only method in this list that also has the option of homogenizing without a reference. The reference series, where used, has to be computed by the operator. It includes a test for documented breakpoints. Note that these tests are not designed for use with any automatic procedure.

SNHT refers to an R-package on CRAN, which implements the well-known Standard Normalized Homogeneity Test in a modified way by Haimberger (2007) and in the pairwise approach used by Menne and Williams (2007).

PHA, pairwise homogenisation algorithm, is used by NOAA to homogenise their national (USHCN) and global temperature dataset (GHCN). Being tested on such a large dataset and using the pairwise approach it is highly robust and recommended for large datasets. It can use metadata.

Table 1.Overview characteristic of homogenisation packages. Next page 


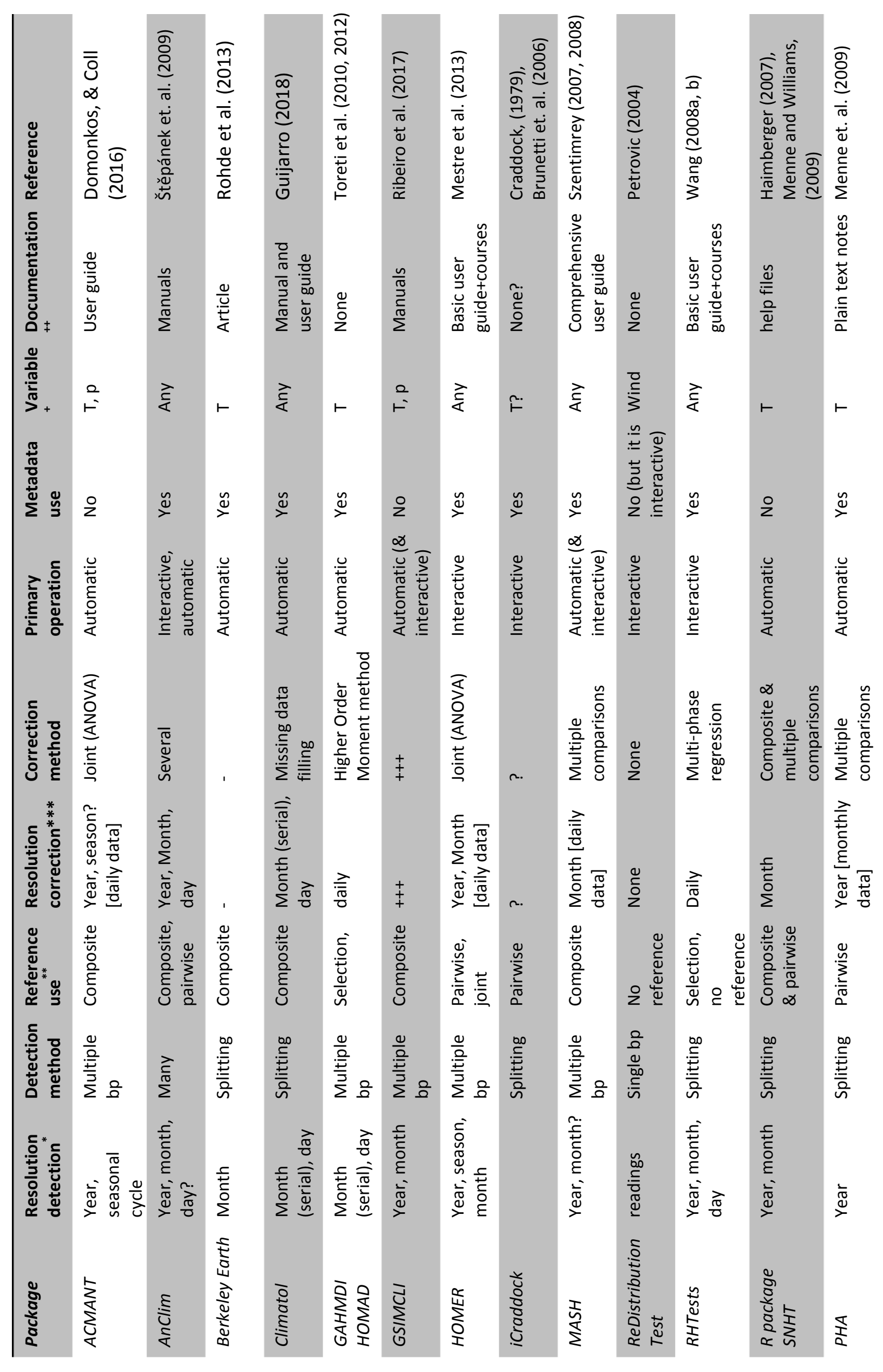


* ACMANT can detect breaks on two series in parallel: the yearly averages and the magnitude of the seasonal cycle. If not noted otherwise in the column Resolution, "month" mean detection is on multiple monthly series in parallel.

** Operator reference selection, averaging (composite reference), removing references with breaks from composite, iteration, pairwise, joint detection.

*** Square brackets mean that the resolution is supported by the software, but the corrections are not computed at that resolution.

${ }^{+} \mathrm{T}$ : temperature; $p$ : precipitation; any: Gaussian and log-normal distribution or additive and multiplicative models; humidity may be difficult.

${ }^{++}$A User guide is limited to a few pages and shorter than a manual.

+++ The corrections are computed at the resolution of the data (annual or monthly series). Corrections are applied to a metric computed by gsimcli (user-defined: percentile, mean or median) of the probability density function (pdf) of the candidate station, which is estimated using composite references.

\subsection{Performance statistical homogenisation}

There are two indications to evaluate whether homogenisation improved a dataset. First, the breaks found should fit to the breaks known from the station history. Second, no more breaks would be detected in the dataset if it were homogenised again and that the results are regionally coherent, physically consistent and climatologically plausible. However, these assessments do not protect against setting too many breaks and removing real regional climate variability (overhomogenisation). In addition, these indications are not strong enough that this helps in selecting the best statistical homogenisation method. We thus mostly rely on what is known about the performance of homogenisation methods from the scientific literature for general cases.

There are two lines of evidence on the general performance of statistical homogenisation methods: theoretical principles and numerical studies. They strengthen each other and to gain confidence we need both. The design principle of a homogenisation method may be sound, but details matter and it may still perform poorly in a numerical comparison. Conversely, numerical studies only test for specific scenarios, which may not be realistic (for the task at hand) and our understanding helps to know what is important and needs to be realistic.

\subsubsection{Theoretical principles}

If a normally distributed uncorrelated difference (candidate minus reference) series may contain one break at a known date the appropriate test is a simple t-test for the difference in the mean before and after the break. If the same series is known to contain maximally one break at an unknown position, multiple testing needs to be taken into account and the appropriate test is the Standard Normalised Homogeneity Test (SNHT; Alexandersson, 1986) or the Penalized Maximal t (PMT) test.

However, climate series typically contain more than one break and also the reference series may contain breaks, which should not be falsely attributed to the candidate series. How statistical homogenisation methods solve these two problems seems to be the main determinant of the performance of statistical homogenisation methods.

There are three ways to detect multiple breakpoints in one series. 
1. Sometimes single-breakpoint tests are performed over moving windows. However, to reduce the probability that there are not multiple breakpoints in the window its length needs to be short. This makes the method less sensitive and this approach is not much used. ${ }^{3}$

2. Traditionally, single-breakpoint methods are used and the series is split at the most significant breakpoint, after which the two new series are tested again (hierarchical splitting and variants thereof).

3. Modern multiple-breakpoint methods (effectively) test all possible multiple break combinations.

Theoretically it is expected that multiple breakpoint methods are the most accurate.

There are also several approaches to handle the problem that the reference series contains inhomogeneities.

1. Averaging over a large number of reference stations. This removes large obvious jumps in the composite reference series, but it often happens that a large part of a network experiences a similar transition over a few years or decades. The bias due to this transition would also largely be in such a composite reference and may reduce detection power.

2. Selecting reference stations without a break around the break in the candidate station or, alternatively, to correct the breaks in the references before they are used as reference. These approaches have to be executed iteratively as the breaks in the references need to be known. Using previously homogenised data is potentially dangerous. Thus this type of methods needs to be validated extra carefully whether they remove the large-scale biases.

3. Detection of breaks on pairs of stations. Here the reference station is not assumed to be homogeneous, with breaks in both stations being detected as breaks in the difference series between them. A second "attribution" step is necessary to determine which of the breaks detected in the pairs belong to which station.

4. Joint detection of all breaks in a network of multiple stations simultaneously. This is a complicated combinatorial puzzle and computationally more demanding than the other methods.

Joint detection is theoretically the optimal solution. However, at the moment the only implementation of this method is in the homogenisation package HOMER, which does not work well by itself.

Traditionally breaks are corrected one station at a time and one break after the other by estimating the size of the jump using a reference series or the median of multiple reference series in case of pairwise methods. This may lead to an accumulation of errors.

A modern joint correction method (often called ANOVA) has been developed by Caussinus and Mestre (2004). This method decomposes a network of stations into one regional climate signal, a step function for every station to model the inhomogeneities and noise. Corrections are computed by minimizing the noise. The corrections of this methods are unbiased if all breaks are correctly identified. Thus all breaks should be set, also breaks that are close to each other. This method has been shown to produce more accurate results than traditional methods for the dense European networks simulated in HOME (Domonkos et al., 2013).

\footnotetext{
${ }^{3}$ A moving-window detection can be useful when you only want to remove clear breaks, but not gradual inhomogeneities. For example, to study the gradual (nonlinear) warming due to urbanization and want to remove the effect of relocations (Zhang et al., 2014).
} 


\subsubsection{Numerical studies}

Traditionally validation studies have focussed on break detection scores of the detection methods. This can help in understanding how the algorithm works, but it is not clear what the optimal compromise for climatological analysis of the homogenised data between the hit rate and the alarm rate is. More recent work has included error measures that assess the performance of entire homogenisation methods and are of relevance to climatological users of the data, such as the root mean square error and the remaining uncertainty in the trend after homogenisation (Domonkos, 2011; Venema et al., 2012; Williams et al., 2012).

To some extent, the results of validation studies will depend on the metric(s) used for evaluation. The way in which homogenisation is carried out may also influence the results (for example, whether metadata was used, how the algorithms was operated and how well-trained the operator was ). In case of manual and semi-automatic methods clear differences between operators were found (Venema et al., 2012) and one should clearly distinguish between the method/package and the contribution validated. Especially for RHtests, which implements several detection tests and correction methods, which can be used in many ways, the results of such a validation study may not be representative.

Williams et al. (2012) only studied the pairwise homogenisation algorithm, Domonkos (2011) compared a large number of automatic homogenisation algorithms, while the COST Action HOME (Venema et al., 2012) included nearly all state-of-the-art and most-used methods, including several manual methods. Note that both studies were performed for dense networks, the performance of the method will be lower for sparser networks. Also the ranking of the methods could be different for other networks, but we would not know how.

These numerical results support the idea that algorithms that are designed to solve the multiplebreakpoint problem and the inhomogeneous-reference problem are able to obtain accuracies that are clearly higher than traditional methods. HOME recommended the algorithms: ACMANT, iCraddock, MASH and PRODIGE and for large networks the Pairwise homogenisation Algorithm.

These validation studies involved the generation of an artificial station network where the inhomogeneities are known. The HOME benchmark dataset aimed to model networks for temperature and precipitation for Europe. Its validation data is quite realistic, but the break variance is about two times too high. The study did not include explicit large-scale trend biases; they were thus small and difficult to correct. Furthermore, its high station density is not representative for the early instrumental period or networks in developing countries. An upcoming benchmarking study of the International Surface Temperature Initiative (ISTI; Thorne et al., 2011) aims to resolve these issues (Willett et al, 2014). New validation results are been obtained in the frame of the MULTITEST project at the time of writing this guide. ${ }^{4}$

In the HOME benchmark all contributions made the temperature data more homogeneous, except for the contribution using absolute homogenisation. This illustrates how dangerous absolute methods can be, see Section 3.4.3, but it should be noted that to make the benchmark blind, the regional climate signal in HOME was more variable than usual and that this dataset was thus more difficult than a real case for absolute homogenisation. Only the best methods were able to improve the homogeneity of precipitation data.

\footnotetext{
${ }^{4}$ http://www.climatol.eu/MULTITEST/)
} 
Validation studies can also be based on high-quality homogenised data. For example in Gubler et al. (2018) information on inhomogeneities based on data homogenised with high station density was used to study the performance of homogenisation methods applied to a thinned sparser network. The advantage of this is that the inhomogeneities are by definition realistic. The problem is that also with a high station density homogenisation will not be perfect. This kind of studies provide synergy together with those using simulated data. Gubler et al. studied four different ways to operate HOMER and found that HOMER using joint detection should not be used by itself. The way the metadata was used, it did not improve the results.

Kuglitsch et al. (2012) also used homogenised Swiss data to validate the homogenisation methods RHTestsV3, PRODIGE and Toreti. PRODIGE finds more breaks, but because only break detection was studied, it is not clear whether it is more sensitive or overestimates the number of breaks, as well as whether the differences in detection matter for the homogeneity of the data.

Numerical results (Domonkos et al., 2013) support the expectation that the joint correction method is indeed an improvement. It could improve the results of all contributions but one to the HOME benchmarking study that did not use it yet.

\subsection{Automated and manual methods}

homogenisation methods may be fully automated (that is, no human intervention is required beyond the selection of the dataset), or they may involve some level of manual intervention. Areas which may involve human judgement in manual methods include:

- The selection of reference stations to be used (whereas an automatic method may use, for example, a purely distance-based criterion).

- Merging information from statistical methods and metadata.

- Determining which inhomogeneities identified by a statistical method should be retained.

- Determining which period to use for a comparison (for example, not using a climatically anomalous year or a less reliable data period in comparing two sites with each other).

Both automatic and manual methods have been used successfully in many countries and the various software systems linked through the Task Team portal contain examples of both. Manual methods do have the advantage of being able to introduce information about a station that may not be easily quantifiable (for example, known gradual changes in the local site environment), and also allow more ready identification of anomalous results at individual stations (although the risk of these is reduced if some of the practices described in section 3.10.

However, manual methods do have the disadvantage of being labour-intensive, and requiring expert judgement, which may not be available in some cases. Furthermore, because it is easy to compute many cases and settings automatic methods can be better validated. This promotes to faster improvements in the capabilities of automatic methods. Furthermore, this also means that we have more reliable estimates of the uncertainties. Additionally removing uncertainties due to human factors means that you are more likely to get the accuracy expected from validation studies..

Automated or semi-automated methods are recommended for when the above four bullet points are not applicable and for users with limited experience in homogenisation. They are also the only practical option for very large datasets, especially global or regional datasets. 


\subsection{Example use cases}

The performance of the homogenisation methods mentioned in the previous section is an important consideration, which will clearly influence the quality of your homogenised data, although most of the listed methods in this chapter will at least typically improve temperature data under most conditions. Further considerations are whether the package can handle metadata, in the case where you have a decent amount of metadata. The size of the network is important; the larger it is the more automatic methods are preferred.

Also the availability of local expertise or training opportunities are a reason to prefer a specific method; for the HOME benchmark clear differences between contributions with the same method, but different operators were found, which may be related to experience with the method. Automatic methods are less influenced by the availability of such expertise and are recommended for inexperienced users.

For a small network with fewer than 50-100 stations, some knowledge of the station history and time to work on a good dataset, both the pairwise method in HOMER and iCraddock are good options. Advantages of HOMER over iCraddock are that the positions of the breaks in the pairs are determined objectively, which also speeds up the task, and HOMER natively supports joint correction. In case the network is sparse and the references may have slightly different climate signals, iCraddock is a good option because the operator can assess graphically whether any differences are climatologically to be expected or reason to suspect an inhomogeneity. In the case where interactive options are not used, MASH would perform similarly to HOMER.

When the network is too sparse and there are effectively no references that can be used (including the availability of reanalysis or proxy series), the only option is to use absolute homogenisation. Such a method is implemented in RHTests.

For mid-sized networks, the automatic methods ACMANT, Climatol and MASH are attractive options. ACMANT can handle networks of up to 100 stations, MASH up to 500, and Climatol network size is only limited by the available memory in the computer. If the size of the network allows for these methods, Climatol and MASH would be preferred in the case where metadata are available, otherwise ACMANT. It should be noted here that one can also split a large network up into multiple smaller ones.

In the above cases the operator carefully selects the series to be used for homogenisation. For very large networks (hundreds or thousands of stations) this becomes cumbersome. The Pairwise homogenisation Algorithm was made for continental and global datasets and can handle messy datasets with short series and missing data.

In case one only wants to check a single breakpoint in a difference time series at a known time, the ttest implemented in RHTests and many other computational packages can be used. 


\section{PART II \\ Advanced topics}




\section{CHAPTER 5 \\ History of homogenisation}

Problems due to inhomogeneities have long been recognised and homogenisation has a long history. In September 1873, at the "International Meteorologen-Congress" in Vienna, Carl Jelinek requested information on national multi-annual data series (k.k. Hof- und Staatsdruckerei, 1873), but decades later, in 1905 G. Hellmann (k.k. Zentralanstalt für Meteorologie und Geodynamik, 1906) still regretted the absence of homogeneous climatological time series due to changes in the surrounding of stations and new instruments and pleaded for stations with a long record, "Säkularstationen", to be kept as homogeneous as possible.

Although this "Conference of directors" recommended maintaining a sufficient number of stations under unchanged conditions today these basic inhomogeneity problems still exist.

\subsection{Detection and adjustments}

Homogenisation has a long tradition. For example, in early times documented change points have been removed with the help of parallel measurements. Differing observing times at the astronomical observatory of the k.k. University of in Vienna (Austria) have been adjusted by using multi-annual 24 hour measurements of the astronomical observatory of the k.k. University of Prague (today Czech Republic). Measurements of Milano (Italy) between 1763 and 1834 have been adjusted to 24 hour means by using measurements of Padova (Kreil, 1854a, 1854b).

However, for the majority of breaks we do not know the break magnitude; furthermore it is most likely that series contain undocumented inhomogeneities as well. In the early $20^{\text {th }}$ century Conrad (1925) applied and evaluated the Heidke criterion (Heidke, 1923) using ratios of two precipitation series. As a consequence he recommended the use of additional criteria to test the homogeneity of series, dealing with the succession and alternation of algebraic signs, the Helmert criterion (Helmert, 1907) and the "painstaking" Abbe criterion (Conrad and Schreier, 1927). The use of Helmert's criterion for pairs of stations and Abbe's criterion still has been described as appropriate tool in the 1940s (Conrad 1944). Some years later the double-mass principle was popularised for break detection (Kohler, 1949).

\subsection{Reference series}

Julius Hann (1880, p. 57) studied the variability of absolute precipitation amounts and ratios between stations. He used these ratios for the quality control. This inspired Brückner (1890) to check precipitation data for inhomogeneities by comparison with neighbouring stations; he did not use any statistics.

In their book "Methods in Climatology" Conrad and Pollak (1950) formalised this relative homogenisation approach, which is now the dominant method to detect and remove the effects of artificial changes. The building of reference series, by averaging the data from many stations in a relatively small geographical area, has been recommended by the WMO Working Group on Climatic Fluctuations (WMO, 1966).

The papers by Alexandersson (1986) and Alexandersson and Moberg (1997) made the Standard Normal Homogeneity Test (SNHT) popular. The broad adoption of SNHT was also for the clear guidance on how to use this test together with references to homogenize station data. 


\subsection{Modern developments}

SNHT is a single-breakpoint method, but climate series typically contain more than one break. Thus a major step forward was the design of methods specifically designed to detect and correct multiple change-points and work with inhomogeneous references (Szentimrey, 1999; Mestre, 1999; Caussinus and Mestre, 2004). These kind of methods were shown to be more accurate by the benchmarking study of the EU COST Action HOME (Venema et al., 2012).

The paper by Caussinus and Mestre (2004) also provided the first description of a method that jointly corrects all series of a network simultaneously. This joint correction method was able to improve the accuracy of all but one contribution to the HOME benchmark that was not yet using this approach (Domonkos et al., 2013).

The ongoing work to create appropriate datasets for climate variability and change studies promoted the continual development of better methods for change point detection and correction. To follow this process the Hungarian Meteorological Service started a series of "Seminars for Homogenization" in 1996 (HMS 1996, WMO 1999, OMSZ 2001, WMO 2004, WMO 2006, WMO 2010). 


\section{CHAPTER 6}

\section{Theoretical background of homogenisation}

This chapter considers some theoretical aspects of monthly series homogenisation. It revisits many homogenisation problems mentioned in part I in more depth. This chapter is intended for users that are interested in a better analytical understanding and for scientists who would like to start developing their own methods.

In practice the monthly series are homogenised mostly in the mean. The aim of these homogenisation procedures is to detect inhomogeneities of the mean and to adjust the series.

For the detection of inhomogeneities of monthly data and their adjustment, solutions are needed for the following mathematical problems:

- statistical spatiotemporal modelling of the series,

- methodology for comparison of candidate and reference series,

- breakpoint (change point) and outlier detection, and

- methodology for adjustment of series.

This chapter will furthermore discuss these topics:

- quality control procedures,

- missing data completion,

- usage of metadata,

- manual versus automatic methods, and

- evaluation of methods (theoretical, benchmark).

\subsection{General structure of the additive spatiotemporal models}

The statistical spatiotemporal modelling of the series is a fundamental question. The adequate comparison, breakpoint detection and adjustment procedures depend on the chosen statistical model.

If the data series are normally distributed (e.g. temperature) then the additive spatiotemporal model can be used. The general form of this additive model for the monthly series of more stations in a small climate region can be written as follows,

$X_{j, m}(t)=\mu_{m}(t)+S_{j, m}+I H_{j, m}(t)+\varepsilon_{j, m}(t),(j=1,2, \ldots, N ; m=1,2, . ., 12 ; t=1,2, \ldots ., n)$,

where

$$
\begin{aligned}
& j=1,2, \ldots, N: \text { index of station } \\
& m=1,2, ., 12: \text { index of month } \\
& t=1,2, \ldots, n: \text { year }
\end{aligned}
$$

$\mu_{m}(t)$ : $\quad$ common and unknown climate change signal or temporal expected values or temporal trend of the stations without any assumption about the shape

$$
\begin{array}{ll}
S_{j, m}: & \quad \text { spatial expected values or spatial trend of the stations } \\
I H_{j, m}(t): \quad & \begin{array}{l}
\text { inhomogeneity signals with type of 'step-like function' in general with unknown } \\
\text { breakpoints } T \text { and shifts } \\
\text { assumed in general. }
\end{array}
\end{array}
$$

Consequently the expected values or means are, 


$$
\mathrm{E}\left(X_{j, m}(t)\right)=\mu_{m}(t)+S_{j, m}+I H_{j, m}(t),(j=1,2, \ldots, N ; m=1,2, . ., 12 ; t=1,2, \ldots ., n)
$$

and superimposed on these means are normal noise series, written in vector form,

$$
\boldsymbol{\varepsilon}_{m}(t)=\left[\varepsilon_{1, m}(t), \ldots . ., \varepsilon_{N, m}(t)\right]^{\mathrm{T}} \in N\left(\mathbf{0}, \mathbf{C}_{m}\right), \quad(m=1,2, . ., 12 ; t=1,2, \ldots ., n) .
$$

The matrices $\mathbf{C}_{m}(m=1,2, . ., 12)$ include the spatial covariances between the stations and they are assumed to be without any climate change or inhomogeneity during the years. It is the consequence of the fact that the existing methods developed for homogenisation of monthly series have the assumption that there is no climate change or inhomogeneity in the higher order moments than the first one.

The vector noise terms $\boldsymbol{\varepsilon}_{m}(t)$ in general also have some temporal auto-correlations, this question is detailed at sections 1.1, 1.2.

The aim of the homogenisation is to detect the inhomogeneity signal $I H_{j, m}(t)$ and to adjust the original raw monthly series $X_{j, m}(t)$ that is,

$X_{H, j, m}(t)=X_{j, m}(t)-I \hat{H}_{j, m}(t) \quad(j=1,2, \ldots, N ; m=1,2, . .12 ; t=1,2, \ldots, n)$,

where $I \hat{H}_{j, m}(t)$ is the estimated inhomogeneity signal.

\section{Remark 1}

In the practice there is some distinction between the methods i.e. absolute or relative ones.

Absolute homogenisation: only one station data series is used i.e. $N=1$. The main problem of the absolute methods is that the separation of the unknown climate change and variability signal $\mu_{m}(t)$ and the inhomogeneity $I H_{j, m}(t)$ is essentially impossible without additional information on the variability of the climate signal for which we would need homogeneous data.

Relative homogenisation: more station data series are used and compared to each other, i.e. $N>1$. The comparison makes it possible to filter out the common unknown climate change and variability signal $\mu_{m}(t)$.

The two basic strategies in order to solve the problem of homogenisation are as follows.

1.1 The monthly series (1) are examined serially as one time series according to the calendar order. At this type of examination the special problems to be solved are,

- annual cycle or seasonality of $\mu_{m}(t), E_{j, m}, I H_{j, m}(t), \mathbf{C}_{m}(m=1,2, . ., 12)$ the latter covariances include also the standard deviations and spatial correlations implicitly,

- moreover the temporal autocorrelation between the elements of the adjacent months.

1.2 The monthly series or the seasonal series or the annual series may be examined independently in parallel. Then the examined series may be,

- the derived monthly series in each calendar month $(m=1,2, . ., 12)$ separately, $X_{j, m}(t)=\mu_{m}(t)+S_{j, m}+I H_{j, m}(t)+\varepsilon_{j, m}(t) \quad(j=1,2, \ldots, N ; t=1,2, \ldots, n)$

- the derived seasonal series in each calendar season $(s=1,2,3,4)$ separately, $X_{j, s}(t)=\mu_{s}(t)+S_{j, s}+I H_{j, s}(t)+\varepsilon_{j, s}(t) \quad(j=1,2, \ldots, N ; t=1,2, \ldots ., n)$

- $\quad$ and the derived annual series, 


$$
X_{j, y}(t)=\mu_{y}(t)+S_{j, y}+I H_{j, y}(t)+\varepsilon_{j, y}(t) \quad(j=1,2, \ldots, N ; t=1,2, \ldots, n)
$$

For this type of examination there is no need to consider the annual cycle or the seasonality and the temporal autocorrelation. In case of a fixed month or fixed season or year it can be accepted that the elements of vector series $\boldsymbol{\varepsilon}_{m}(t)(t=1, . ., n)$ or $\boldsymbol{\varepsilon}_{s}(t)(t=1, \ldots n)$ or $\boldsymbol{\varepsilon}_{y}(t)(t=1, \ldots n)$ are totally independent in time. However after the parallel examinations a synthesis is necessary for the estimated monthly inhomogeneity signals $I \hat{H}_{j, m}(t)(m=1,2, . ., 12)$. If the data series are quasi lognormal distributed (e.g. precipitation) then a multiplicative model can be used that can be transformed into an additive by applying a logarithmic transformation procedure.

\subsection{Methodology for comparison of series in case of additive model}

In the relative homogenisation approach a chosen candidate station series is compared to the other station series as reference series. That removes the common climate change and variability signal, which is unknown.

Using the notations (1) all the examined station series $X_{j, m}(t)(j=1, \ldots, N)$ have to be taken as candidate and reference series alike, furthermore the reference series are not assumed to be homogeneous at the correct examinations since there is no information about it in general. The main aim of the comparison is to filter out the unknown climate change signal $\mu_{m}(t)$.

The problem of comparison of series incorporates the following topics:

- pairwise comparison,

- composite reference series creation,

- difference series constitution, and

- multiple comparisons of series.

These topics are very important for the detection as well as for the adjustment, because efficient series comparison can increase both detection power and correction accuracy. The development of efficient comparison methods can be based on the examination of the spatial covariance structure of data series.

The pairwise difference series between pairs are $Z_{j, m}(t)=X_{j, m}(t)-X_{i, m}(t)$. However, the difference series constitution can be formulated in a more general way as well.

Assuming that $X_{j, m}(t)$ is the candidate series and the other ones are the reference series, then the difference series belonging to the candidate series can be constituted as,

$Z_{j, m}(t)=X_{j, m}(t)-\sum_{i \neq j} \lambda_{j i, m} X_{i, m}(t)=I H_{j, m}(t)-\sum_{i \neq j} \lambda_{j i, m} I H_{i, m}(t)+\varepsilon_{Z_{j, m}}(t)$

where $\sum_{i \neq j} \lambda_{j i, m} X_{i, m}(t)$ is a composite reference series with condition of $\sum_{i \neq j} \lambda_{j i, m}=1$ for the weighting factors. As a result of the last condition, the unknown climate change and variability signal $\mu_{m}(t)$ has been filtered out. Consequently the inhomogeneity can be detected by the examination of the above difference series. Since the difference series (2) includes inhomogeneities from candidate and reference in general, it may be useful to select reference series with better quality and to create several composite reference series for a candidate series. Multiple comparison or examination of multiple difference series can facilitate the attribution of the appropriate detected inhomogeneity for the candidate series. 
There are three considerations to select or weight reference series. 1) reduce the noise of the difference series, 2) reduce the influence of inhomogeneities in the reference, 3) make sure that the reference series has a similar regional climate signal.

To increase the signal to noise ratio in order to increase the power of detection then we have to decrease the variance of the noise term ${ }^{Z_{j, m}}(t)$. The optimal weighting factors $\lambda_{j i, m}$ that minimize the variance are determined by the spatial covariance matrices $\mathbf{C}_{m}$ uniquely (optimal interpolation or kriging weights). To reduce the influence of inhomogeneities in nearby stations, which get large kriging weights, it may be desirable to average over more stations or give further away stations a relatively stronger weight. On the other hand, to make sure that all stations experience the same regional climate signal it may be desirable to limit the number of stations in sparse networks.

It can be proved mathematically that using the maximum likelihood principle for the joint detection or/and the joint adjustment assuming normal distribution then also certain difference series are examined indirectly where the weighting factors of the reference series are determined by the spatial covariance matrices. We will return to this question at section 6.3.1 of Bayesian approach (model selection); penalized likelihood methods.

\subsection{Methodology for breakpoint (changepoint) detection}

One of the basic tasks of the homogenisation is the examination of the difference series (Eq. 2 ) in order to detect the breakpoints and to attribute the appropriate ones for the candidate series.

The scheme of the breakpoint detection is as follows. Let $Z(t)$ be a difference series according to the formula (2), that is

$$
Z(t)=I H_{Z}(t)+\varepsilon_{Z}(t) \quad(t=1, . ., n),
$$

where $I H_{Z}(t)$ is a mixed inhomogeneity of difference series $Z(t)$ with breakpoints from candidate and references. In general the number of breakpoints and their positions with sizes are unknown and we assume $\varepsilon_{Z}(t)$ is a normal noise series. In case of the parallel homogenisation (item 1.2) $\varepsilon_{Z}(t)$ is a normal white (uncorrelated) noise series since their elements are assumed to be independent in time.

Remark 2 (Outlier detection, QC)

Outlier detection is the main quality control (QC) procedure for the monthly data in this process. If an outlier is not removed it may produce two neighbouring break points where their sizes are the same in absolute value, but with opposite sign.

Returning to the detection procedures the basic types are the hierarchical splitting (stepwise detection) and the multiple breakpoints detection. The hierarchical splitting procedure is a repeated single-breakpoint detection. The multiple breakpoint detection procedures were developed for the estimation of all breakpoints in a candidate series.

There may be different principles of the detection methods that are classical methods in the mathematical statistics.

\subsubsection{Breakpoint detection based on Maximum Likelihood Estimation}

The methods based on Bayesian model selection (segmentation) are the penalized likelihood methods. Then joint maximum likelihood estimation is given for the breakpoints assuming normal distribution of the difference series and using some penalty term. The reason of the penalty term is that the number of breakpoints is unknown. The methods may be different in the penalty terms or 
criterions e.g. Akaike criterion, Schwarz criterion, Caussinus-Lyazrhi criterion. The penalty terms depend on some 'a priori' probability of break at each time.

Theoretically this methodology could be also applied for the joint detection of the breakpoints of all the examined series. However the joint likelihood function assuming normal distribution depends on the inverse of the spatial covariance matrix what can cause complicated technical problems in case of larger networks. We have also the non-trivial statistical model problem to be solved that the climate signals may be different in case of larger networks.

\subsubsection{Breakpoint detection based on Test of Hypothesis}

Another way is to use test of hypothesis methods for the detection of breakpoints of the difference series. The null hypothesis is homogeneity. These methods also assume the normal distribution therefore the test statistics are derived from the t-type statistics in general. In case of no reference is used (absolute homogenisation) F-type statistics are derived because of the regression of unknown temporal trend. The significance and the power of the procedures can be defined according to the probabilities of the two types of errors. Type one errors are detecting false breakpoints, while type two errors are missing real breakpoints. A compromise between these two types of errors has to be found. The test statistics can be compared to the critical value that depends on the given significance level. In case of multiple test statistics the critical values can be calculated by Monte Carlo methods.

Most of these methods are stepwise ones which are repeated single-breakpoint detection. However, multiple breakpoint detection procedures also can be developed. The essence of this procedure is that between the neighbouring detected breakpoints the homogeneity can be accepted and between non-neighbouring detected breakpoints the homogeneity cannot be accepted. In addition confidence intervals also can be given for the breakpoints that make it possible to make use of metadata automatically.

It is a favourable property of these methods that the result can be evaluated, and validated by comparison of the test statistics before and after homogenisation.

\subsubsection{Attribution of the detected breakpoints for the candidate series}

During the breakpoints detection procedures the difference series are examined with mixed inhomogeneities (Eq. 2). There are two basic types of the methods in order to solve this problem.

i) If only one difference series is examined for a candidate series then all the detected breakpoints are attributed to the candidate series. Then the possible inhomogeneities in the references are a very serious problem. Therefore it is necessary to select more reference series with better quality for a composite reference series to be created expectedly without breakpoints.

ii) If more difference series are examined for a candidate series then the mixed inhomogeneity is less of a problem, but the attribution of the breakpoints to the candidate series is not a trivial task. Then a synthesis is necessary and the key question of the homogenisation software is to develop automatic procedures for this attribution problem.

\subsection{Methodology for adjustment of series}

Beside detection another basic task is the adjustment of series. Calculation of the adjustment factors can be based on the examination of difference series for estimation of shifts at the detected break points. In general the methods use point estimation for the shifts at the detected break points.

There are methods that use the standard least squares technique after breakpoint detection procedure for joint estimation of the shifts of all the examined series. Probably the generalized least squares estimation technique based on spatial covariance matrix could be more efficient and it would be equivalent with the maximum likelihood estimation for the shifts in the case of normal distribution. However we have the non-trivial statistical model problem to be solved that the climate signals may be different in case of larger networks. 
Another way is that the calculation of the adjustment factors is based on some confidence intervals given for the shifts at the detected break points. The confidence intervals given for the break points and shifts make possible also the automatic use of metadata.

Remark 3 (missing data)

The missing data completion or filling the gaps is essentially an interpolation problem. To use the spatial covariances for the calculation of the weighting factors of the predictors is strongly recommended in order to decrease the interpolation error.

\subsection{Possibilities for evaluation, validation of the methods}

If want to obtain a real image of the methods, then the theoretical evaluation of their mathematical basis is indispensable.

Another possibility is a blind comparison and validation study for the homogenisation methods. Then the methods are tested on a realistic benchmark dataset. The benchmark contains simulated data with inserted inhomogeneity. Testing the methods on a generated benchmark dataset seems to be an objective validation procedure, however, we have to know also the limits of such type of examinations.

The interpretation of benchmark results is not a trivial problem, since these are depending on different factors, such as:

- tested methods (quality, manual or automatic),

- testing benchmark dataset (quality, adequacy),

- testers (skilled or unskilled),

- methodology of evaluation (validation statistics).

The creation of adequate benchmark dataset and the development of appropriate validation statistics are critical points and they need also strong theoretical mathematical background. For example, to understand what statistical characteristics of a benchmark are important and need to be modelled realistically and require more detailed studies to know what range is realistic in real networks.

One of the advantages of automatic homogenisation methods is that they lend themselves better to objective validation for two reasons. 1) It is less work to homogenize large datasets needed to see whether new developments lead to statistically significant improvements. 2) In case of manual methods not only the method, but also the operator is assessed, which may bias the results in either direction. 


\section{CHAPTER 7}

\section{Future research \& collaboration needs}

This guidance is based on our current best understanding of inhomogeneities and homogenisation. However, there is a clear need for a better understanding of the problems.

A better mathematical understanding of statistical homogenisation is important because that is what most of our work is based on. A stronger mathematical basis is a prerequisite for future methodological improvements.

A stronger focus on a (physical) understanding of inhomogeneities would complement and strengthen the statistical work. This kind of work is often performed at the station or network level, but also needed at larger spatial scales. Much of this work is performed using parallel measurements, but they are typically not internationally shared.

In an observational science the strength of the outcomes depends on a consilience of evidence. Thus having evidence on inhomogeneities from both statistical homogenisation and physical studies strengthens the science.

This chapter will discuss the needs for future research on homogenisation grouped in five kinds of problems. In the first section we will discuss research on improving our physical understanding and physics-based corrections. The next section is about break detection, especially about two fundamental problems in statistical homogenisation: the inhomogeneous-reference problem and the multiple-breakpoint problem.

Section 3 will be about computing uncertainties in trends and long-term variability estimates from homogenised data due to remaining inhomogeneities. It may be possible to improve correction methods by treating it as a statistical model selection problem (Section 4). The last section discusses whether inhomogeneities are stochastic or deterministic and how that may affect homogenisation and especially correction methods for the variability around the long-term mean.

For all the research ideas mentioned below goes that we should study variables other than temperature more. In addition, more studies on inhomogeneities across variables could be helpful to understand the causes of inhomogeneities and increase the signal to noise ratio. Homogenisation by national offices has advantages because here all climate elements from one station are stored together. This helps in understanding and identifying breaks. It would help homogenisation science and climate analysis to have a global database for all climate elements, like iCOADS for marine data. A Copernicus project has started working on this for land station data, which is an encouraging development.

\subsection{Physical understanding}

It is good scientific practise make parallel measurements to manage unavoidable changes and to compare the results of statistical homogenisation to the expectations given the cause of the inhomogeneity according to the metadata. This information should also be analysed on continental and global scales to get a better understanding of when historical transitions took place and to guide homogenisation of large-scale (global) datasets. This requires more international sharing of parallel data and standards on the reporting of the size of breaks confirmed by metadata. 
The Dutch weather service KNMI published a protocol how to manage possible future changes of the network, who decides what needs to be done in which situation, what kind of studies should be made, where the studies should be published and that the parallel data should be stored in their central database as experimental data. A translation of this report is attached to this guidance as Appendix A and will hopefully inspire other weather services to formalise their network change management..

Next to statistical homogenisation, making and studying parallel measurements, and other physical estimates, can provide a second line of evidence on the magnitude of inhomogeneities. Having multiple lines of evidence provides robustness to observational sciences. Parallel data is especially important for the large historical transitions that are most likely to produce biases in network-wide to global climate datasets. It can validate the results of statistical homogenisation and be used to estimate possibly needed additional adjustments. The Parallel Observations Science Team of the International Surface Temperature Initiative (ISTI-POST) is working on building such a global dataset with parallel measurements.

Parallel data is especially suited to improve our physical understand of the causes of inhomogeneities by studying how the magnitude of the inhomogeneity depends on the weather and on instrumental design characteristics. This understanding is important for more accurate corrections of the distribution, for realistic benchmarking datasets to test our homogenisation methods and to determine which additional parallel experiments are especially useful.

Detailed physical models of the measurement, for example, the flow through the screens, radiative transfer and heat flows, can also help gain a better understanding of the measurement and its error sources. This aids in understanding historical instruments and in designing better future instruments. Physical models will also be paramount for understanding the influence of the surrounding on the measurement, from nearby obstacles and surfaces influencing error sources and air flow to changes in the regional land use influencing the measurand, such as urbanization in the introduction of irrigation. Land-use changes, especially urbanisation, should be studied together with relocations they may provoke.

\subsection{Break detection}

Longer climate series typically contain more than one break. This so-called multiple-breakpoint problem is currently an important research topic. A complication of relative homogenisation is that also the reference stations can have inhomogeneities. This so-called inhomogeneous-reference problem is not optimally solved yet. It is also not clear what temporal resolution is best for detection and what the optimal way is to handle the seasonal cycle in the statistical properties of climate data and of many inhomogeneities.

For temperature time series about one break per 15 to 20 years is typical and multiple breaks are thus common. Unfortunately, most statistical detection methods have been developed for one break and for the null hypothesis of white (sometimes red) noise. In case of multiple breaks the statistical test should not only take the noise variance into account, but also the break variance from breaks at other positions. For low signal to noise ratios, the additional break variance can lead to spurious detections and inaccuracies in the break position.

To apply single-breakpoint tests on series with multiple breaks, one ad-hoc solution is to first split the series at the most significant break (for example the standard normalized homogeneity test, SNHT) and investigate the subseries. Such a greedy algorithm does not always find the optimal solution. Another solution is to detect breaks on short windows. The window should be short enough to 
contain only one break, which reduces power of detection considerably. This method is not used much nowadays.

Multiple breakpoint methods can find an optimal solution and are nowadays numerically feasible. This can be done in a hypothesis testing (MASH) or in a statistical model selection framework framework. For a certain number of breaks these methods find the break combination that minimize the internal variance, that is variance of the homogeneous subperiods, (or you could also state that the break combination maximizes the variance of the breaks). To find the optimal number of breaks, a penalty is added that increases with the number of breaks. Examples of such methods are PRODIGE (Caussinus \& Mestre, 2004) or ACMANT (based on PRODIGE; Domonkos, 2011). In a similar line of research Lu et al. (2010) solved the multiple breakpoint problem using a minimum description length (MDL) based information criterion as penalty function.

This penalty function of PRODIGE was found to be suboptimal (Lindau \& Venema, 2013a). It was found that the penalty should be a function of the number of breaks, not fixed per break and that the relation with the length of the series should be reversed. It is not clear yet how sensitive homogenisation methods respond to this.

Not only the candidate station, also the reference stations will have inhomogeneities, which complicates homogenisation. Such inhomogeneities can be climatologically especially important when they are due to network-wide technological transitions. An example of such a transition is the current replacement of temperature observations using Stevenson screens by automatic weather stations. Such transitions are important periods as they may cause biases in the network and global average trends and they produce many breaks over a short period.

A related problem is that sometimes all stations in a network have a break at the same date, for example, when a weather service changes the time of observation. Nationally such breaks are corrected using metadata. If this change is unknown in global datasets one can still detect and correct such inhomogeneities statistically by comparison with other nearby networks. That would require an algorithm that additionally knows which stations belong to which network and prioritizes correcting breaks found between stations in different networks. Such algorithms do not exist yet and information on which station belongs to which network for which period is typically not internationally shared.

The influence of inhomogeneities in the reference can be reduced by computing composite references over many stations, removing reference stations with breaks and by performing homogenisation iteratively.

A direct approach to solving this problem would be to simultaneously homogenise multiple stations, also called joint detection. A step in this direction are pairwise homogenisation methods where breaks are been detected in the pairs. This requires an additional attribution step, which attributes the breaks to a specific station. Currently this is done by hand (for PRODIGE; Caussinus and Mestre, 2004) or with ad-hoc rules (by the Pairwise homogenisation algorithm of NOAA; Menne and Williams, 2009).

In the homogenisation method HOMER (Mestre et al., 2013) a first attempt is made to homogenize all pairs simultaneously using a joint detection method from bio-statistics. Feedback from first users suggests that this method should not be used automatically. It should be studied how good this methods works and where the problems come from. 
Multiple breakpoint methods are more accurate as single breakpoint methods. This expected higher accuracy is founded theoretically (Hawkins, 1972). In addition, in the HOME benchmarking study it was numerically found that modern homogenisation methods, which take the multiple breakpoint and the inhomogeneous reference problems into account, are about a factor two more accurate as traditional methods (Venema et al., 2012).

However, the current version of CLIMATOL applies single-breakpoint detection tests, first SNHT detection on a window then splitting, to achieve results comparable to modern multiple-breakpoint methods with respect to break detection and homogeneity of the data (Killick, 2016). This suggests that the multiple-breakpoint detection principle may not be as important as previously thought and warrants deeper study.

The signal to noise ratio is paramount for the reliable detection of breaks. It would thus be valuable to develop statistical methods that explain part of the variance of a difference time series and remove this to see breaks more clearly. Data from (regional) reanalysis could be useful for this.

First methods have been published to detect breaks for daily data (Toreti et al., 2012; Rienzner and Gandolfi, 2013). It has not been studied yet what the optimal resolution for breaks detection is, daily, monthly, annual is, nor what the optimal way is to handle the seasonal cycle in the climate data and exploit the seasonal cycle of inhomogeneities. In the daily temperature benchmarking study of Killick (2016) most annual and monthly detection methods performed better than the daily detection method MAC-D (Rienzner and Gandolfi, 2013).

The selection of appropriate reference stations is a necessary step for accurate detection and correction. Many different methods and metrics are used for the station selection, but studies on the optimal method are missing. The knowledge of local climatologists which stations have a similar regional climate needs to be made objective so that it can be applied automatically (at larger scales).

For detection the reduction of the noise level is most important, for correction it is paramount that all stations are in the same climatic region. Typically the same networks are used for both, but it should be investigated whether a smaller network for correction would be beneficial. Also in general, we need more research on understanding the performance of (monthly and daily) correction methods.

\subsection{Computing uncertainties}

Also after homogenisation uncertainties remain in the data due to various problems.

- Not all breaks in the candidate station have been and can be detected.

- False alarms are an unavoidable trade-off for detecting many real breaks.

- Uncertainty in the estimation of correction parameters due to limited data.

- Uncertainties in the corrections due to limited information on the break positions.

From validation and benchmarking studies we have a reasonable idea about the remaining uncertainties that one can expect in the homogenised data, at least with respect to changes in the long-term mean temperature. For many other variables and changes in the distribution of (sub-)daily temperature data individual developers have validated their methods, but systematic validation and comparison studies are still missing.

Furthermore, such studies only provide a general uncertainty level, whereas more detailed information for every single station/region and period would be valuable. The uncertainties will 
strongly depend on the signal to noise ratios, on the statistical properties of the inhomogeneities of the raw data and on the quality and cross-correlations of the reference stations. All of which vary strongly per station, region and period.

Communicating such a complicated errors structure, which is mainly temporal, but also partially spatial, is a problem in itself. Furthermore, not only the uncertainty in the means should be considered, but, especially for daily data, uncertainties in the complete probability density function need to be estimated and communicated. This could be communicated with an ensemble of possible realizations, similar to Brohan et al. (2006).

An analytic understanding of the uncertainties is important, but is often limited to idealised cases. Thus also numerical validation studies, such as the past HOME and upcoming ISTI studies are important for an assessment of homogenisation algorithms under realistic conditions.

Creating validation datasets also make clear that our understanding of the statistical properties of the break signal is limited. This is especially the case for variables other than temperature and for daily and (sub-)daily data. Information is needed on the real break frequencies and size distributions, but also their auto-correlations and cross-correlations, as well as explained in the next section the stochastic nature of breaks in the variability around the mean.

Validation studies focussed on difficult cases would be valuable for a better understanding. For example, sparse networks, isolated island networks, large spatial trend gradients and strong decadal variability in the difference series of nearby stations (for example, due to El Nino in complex mountainous regions).

The advantage of simulated data is that it can create a large number of quite realistic complete networks. For daily data it will remain hard for the years to come to determine how to generate a realistic validation dataset. Thus even if using parallel measurements is mostly limited to one break per test, it does provide the highest degree of realism for this one break.

\subsection{Deterministic or stochastic corrections?}

Annual and monthly data is normally used to study trends and variability in the mean state of the atmosphere. Consequently, typically only the mean is adjusted by homogenisation. Daily data, on the other hand is used to study climatic changes in weather variability, severe weather and extremes. Consequently, not only the mean should be corrected, but the full probability distribution describing the variability of the weather.

The physics of the problem suggests that many inhomogeneities are caused by stochastic processes. An example affecting many instruments are differences in the response time of instruments, which can lead to differences determined by turbulence. A fast thermometer will on average read higher maximum temperatures than a slow one, but this difference will be variable and sometimes be much higher than the average. In case of errors due to insolation the radiation error will be modulated by clouds. An insufficiently shielded thermometer will need larger corrections on warm days, which will typically be more sunny, but some warm days will be cloudy and not need much correction, while other warm days are sunny and calm and have a dry hot surface. The adjustment of daily data for studies on changes in the variability is thus a distribution problem and not only a regression biascorrection problem. For assimilation accurate bias correction (with regression methods) is probably the main concern. 
Seen as a variability problem, the correction of daily data is similar to statistical downscaling in many ways. Both methodologies aim to produce bias-corrected data with the right variability, taking into account the local climate and large-scale circulation. One lesson from statistical downscaling is that increasing the variance of a time series deterministically by multiplication with a fraction, called inflation, is the wrong approach and that the variance that could not be explained by regression using predictors should be added stochastically as noise instead (Von Storch, 1999). Maraun (2013) demonstrated that the inflation problem also exists for the deterministic Quantile Matching method, which is also used in daily homogenisation. Current statistical correction methods deterministically change the daily temperature distribution and do not stochastically add noise.

Transferring ideas from downscaling to daily homogenisation is likely fruitful to develop such stochastic variability correction methods. For example, predictor selection methods from downscaling could be useful. Both fields require powerful and robust (time invariant) predictors. Multi-site statistical downscaling techniques aim at reproducing the auto- and cross-correlations between stations (Maraun et al., 2010), which may be interesting for homogenisation as well.

The daily temperature benchmarking study of Rachel Killick (2016) suggests that current daily correction methods are not able to improve the distribution much. There is a pressing need for more research on this topic. However, these methods likely also performed less well because they were used together with detection methods with a much lower hit rate than the comparison methods.

The deterministic correction methods may not lead to severe errors in homogenisation, that should still be studied, but stochastic methods that implement the corrections by adding noise would at least theoretically fit better to the problem. Such stochastic corrections are not trivial and should have the right variability on all temporal and spatial scales.

It should be studied whether it may be better to only detect the dates of break inhomogeneities and perform the analysis on the homogeneous subperiods (removing the need for corrections). In case of trend analysis, this would be similar to the work of the Berkeley Earth Surface Temperature group on the mean temperature signal. Periods with gradual inhomogeneities, e.g. due to urbanization, would have to be detected and excluded from such an analysis.

An outstanding problem is that current variability correction methods have only been developed for break inhomogeneities, methods for gradual ones are still missing. In homogenisation of the mean of annual and monthly data, gradual inhomogeneities are successfully removed by implementing multiple small breaks in the same direction. However, as daily data is used to study changes in the distribution, this may not be appropriate for daily data as it could produce larger deviations near the breaks. Furthermore, changing the variance in data with a trend can be problematic (Von Storch, 1999).

At the moment most daily correction methods correct the breaks one after another. In monthly homogenisation it is found that correcting all breaks simultaneously (Caussinus and Mestre, 2004) is more accurate (Domonkos et al., 2011). It is thus likely worthwhile to develop multiple breakpoint correction methods for daily data as well.

Finally, current daily correction methods rely on previously detected breaks and assume that the homogeneous subperiods (HSP) are homogeneous. However, these HSP are currently based on detection of breaks in the mean only. Breaks in higher moments may thus still be present in the "homogeneous" subperiods and affect the corrections. If only for this reason, we should also work on detection of breaks in the distribution. 


\subsection{Correction as model selection problem}

The number of degrees of freedom (DOF) of the various correction methods varies widely. From just one degree of freedom for annual corrections of the means, to 12 degrees of freedom for monthly correction of the means, to 120 for decile corrections applied to every month, to a large number of DOF for quantile or percentile matching.

A study using PRODIGE on the HOME benchmark suggested that for typical European networks monthly adjustment are best for temperature; annual corrections are less accurate probably because they fail to account for changes in seasonal cycle due to inhomogeneities. For precipitation annual corrections were most accurate; monthly corrections were less accurate likely because the data was too noisy to estimate the 12 correction constants/degrees of freedom.

What is the best correction method depends on the characteristics of the inhomogeneity. For a calibration problem just the annual mean could be sufficient, for a serious exposure problem (e.g., insolation of the instrument) a seasonal cycle in the monthly corrections may be expected and the full distribution of the daily temperatures may need to be adjusted. The best correction method also depends on the reference. Whether the variables of a certain correction model can be reliably estimated depends on how well-correlated the neighbouring reference stations are.

An entire regional network is typically homogenised with the same correction method, while the optimal correction method will depend on the characteristics of each individual break and on the quality of the reference. These will vary from station to station, from break to break and from period to period. Work on correction methods that objectively select the optimal correction method, e.g., using an information criterion, would be valuable.

In case of (sub-)daily data, the options to select from become even larger. Daily data can be corrected just for inhomogeneities in the mean or also for the variability around the mean. In between are methods that adjust for the distribution including the seasonal cycle, which dominates the variability and is thus effectively similar mean adjustments with a seasonal cycle. Correction method of intermediate complexity with more than one, but less than 10 degrees of freedom would fill a gap and allow for more flexibility in selecting the optimal correction model.

When applying these methods (Della-Marta and Wanner, 2006; Wang et al., 2010; Mestre et al., 2011; Trewin, 2013) the number of quantile bins (categories) needs to be selected as well as whether to use physical weather-dependent predictors and the functional form they are used (Auchmann and Brönnimann, 2012). Objective optimal methods for these selections would be valuable. 


\section{APPENDIX A}

\section{Protocol Measurement Infrastructure Changes}

This appendix is a translation of the protocol for managing changes in the measurement infrastructure of the Dutch weather service KMNI in De Bilt. The protocol was edited by Theo Brandsma and was published as an internal rapport in 2011 under report number: IR-2011-04. Authors are Theo Brandsma (red.), Aryan van Engelen, Albert Klein Tank, Jitze van der Meulen, Wim Monna and Gerard van der Schrier. It can be found on the KNMI homepage at: http://bibliotheek.knmi.nl/knmipubIR/IR2011-04.pdf 


\section{Content}

\section{Background and purpose}

2 Prerequisites

\section{Changes in the measurement infrastructure}

\section{Communication and general agreements}

\section{Specific agreements for each type of change}

5.1 Relocation of a weather station or relocation of one or more instruments

5.2 Change in standard sensor type, instrument screen or setup

5.3 Change in algorithms/procedures, in data processing systems or in the validation 5.4 Defects or abnormalities in sensors or their setup while measuring

5.5 Changes in the setup and maintenance of the station

5.6 Changes in the instrument maintenance

5.7 Changes in calibration procedures and techniques

5.8 Abrupt or slow changes in the surrounding of the station

5.9 Changes in the protocol for the documentation of metadata

\section{Literature}

\section{Appendix 1: GCOS Essential Climate Variables}

\section{Appendix 2: GCOS Climate Monitoring Principles}

\section{Appendix 3: Abbreviations}




\section{Background and purpose}

Changes in the measurement infrastructure at KNMI are sometimes unavoidable. This often involves a forced relocation (e.g., cancellation of land leases) or relocations due to changes in the nearby terrain surrounding the measurement site. In the latter case, the measurements are often no longer representative of the surroundings. Changes in monitoring infrastructure may also be intended to be more cost efficient or to improve the quality of measurements by, for example, better sensors and/or screens.

Changes in the measurement infrastructure or conditions lead to inhomogeneities ${ }^{5}$ in measurement series. For climate research and applications these artificial (not caused by the climate) jumps or trends are undesirable and should thus be limited as much as possible. It is essential to recognize and quantify such jumps or trends so that it is possible to analyse them. Otherwise an inhomogeneity could be wrongly interpreted as a climatic change.

This Protocol Measurement Infrastructure Changes (PMIC) ensures that (a) inhomogeneities are minimized, and (b) where changes are inevitable, that timely and accurate assessment of the magnitude of any inhomogeneity is made and communicated in the right way. Parallel measure in the 'old' and 'new' situation may be part of this. This makes correction possible, and is thus part of the quality assurance of all climatological observations carried out under the responsibility of the KNMI. In this way, this PMIC contributes to our computation of reliable trends and variability in climate variables on well homogenised series.

The PMIC also helps to implement the GCOS principles of climate monitoring (WMO, 2010; see Appendix 2) at KNMI. GCOS has prepared a list of Essential Climate Variables (ECVs; WMO, 2010; see Appendix 1), this is a list of variables to be observed, which are essential for climate monitoring and research. A number of these variables are relevant for the KNMI. For these this PMIC is applicable. It concerns both land and maritime observations.

TR-278 (Technical Report Wessels, 2005) provides a first step to achieve a PMIC. TR-278 treats the WMO requirements regarding observations, representativeness of the measurements and the WMO recommendations for changes in monitoring infrastructure. TR 278 provides the background for the PMIC described in this document.

\footnotetext{
${ }^{5} \mathrm{~A}$ time series of a climate variable is called homogeneous when the variations in that series are only caused by variations in climate and weather (Conrad and Pollak, 1962). Inhomogeneities are caused by changes in measuring instruments, observation methods and environment (i.e., by local artificial and natural changes, but also by changes in the nearby area).
} 


\section{Prerequisites}

The PMIC expects that the following is fulfilled:

1. Setup and maintenance of the monitoring network shall follow the WMO Standards and Regulations and its implementation in the KNMI Observations Handbook.

2. There is an authorized form of version control system and documentation for algorithms and procedures used for data processing systems, validation, the climate database, etc. This information is centrally available at KNMI Intranet and is archived appropriately and managed.

3. Station metadata is accessible, archived and managed in a special metadata database. 


\section{Changes in the measurement infrastructure}

The following changes in the measurement infrastructure cause inhomogeneities in climate series and are covered in this PMIC:

1. Relocation of the weather station or relocation of one or more instruments outside the measurement site.

2. Change of the sensor type, screen, or the setup of an instrument.

3. Change in algorithms/procedures, data processing systems, in the analysis (e.g., how averages, minimums or maximums are calculated), in the validation, possibly correction or in the archiving of data in climate database (e.g., its resolution).

4. Defects or abnormalities that occurred during measurement in sensors or the setup that were noted due to validation or calibration, as a result of which the required maximum uncertainty is no longer satisfied.

5. Changes in the setup and maintenance of the weather station

6. Changes in the maintenance of instruments.

7. Changes in calibration procedures and techniques.

8. Abrupt or gradual changes in the surrounding of the observing site (both temporary and lasting).

9. Changes in the protocol for documenting metadata. 


\section{Communication and general agreements}

1. The Tactical Meeting on Observations is responsible for the content and management of the PMIC; the Strategic Meeting on Observations authorizes the PMIC.

2. The leader of the Department observing infrastructure is responsible for compliance with the PMIC and the internal communication. External communication is provided by the Department Infrastructure-Information Services.

3. Changes in state ${ }^{6}$ of the measurement infrastructure are reported to all stakeholders ${ }^{7}$ by the leader of the Department observing infrastructure using the same protocol ${ }^{8}$.

4. Per station and per observing system/variable the status can be found on the Intranet (coupled to the Stations Administration and Information System and managed by the Department Infrastructure-Information Services).

5. Usefulness and necessity of a change in the measurement infrastructure are discussed in the Tactical Meeting on Observations (TMO). The TMO decides in consultation with the stakeholders on the plan of action.

6. In the case of parallel measurements, the TMO will agree who is/are responsible for the execution of the measurement, the analysis and reporting. Planning and effort allocation belong to this appointment.

7. Parallel measurements are archived as experimental measurements in the climate database and made available on the Internet with documentation.

8. Implemented changes in the measurement infrastructure are documented and filed in the stations metadata database.

6 The status says something about planned or ongoing changes in the measurement infrastructure, such as the movement of an AWS, the transition to another type of sensor, etc.

7 Stakeholders are all individuals and departments involved in the measurement chain (from sensor up to the climate database) and also include users of the data, which want to stay up to date on the quality of the data. These parties are known to the Department Infrastructure-Information Services.

8 As of May 2011 a communication tool for this will be made available. 


\section{Specific agreements for each type of change}

\subsection{Relocation of a weather station or relocation of one or more instruments}

1. In case of an essential relocation of a complete weather station or of one or more instruments outside the measurement site, parallel measurements will always be performed to examine the effects for KNMI's relevant ECVs. One speaks of an essential relocation if the movement is more than $100 \mathrm{~m}$, or the movement is to a location, which is different in terms of surrounding of the previous one. It is only allowed to deviate from this rule in case of compelling reasons (force majeure) and after assessment by the Tactical Meeting on Observations.

2. Parallel measurements are implemented for a standard period of 2 years. ${ }^{9}$ This period is equal to the WMO directive.

3. The results of the measurements are documented in a public report. The metadata published on the internet noting the change references this report.

4. In case of significant differences between the old and new location, the report indicates whether and how the measurements of the two locations can be combined with one another.

5. The old location will only be terminated after completion of the report mentioned under (4), which makes clear whether the new measurement is considered a continuation of the old station or a new station.

6. When the need arises only the Tactical Meeting on Observations can decide to deviate from the above points.

\subsection{Change in standard sensor type, instrument screen or setup}

1. In case of a change to a new standard sensor type and/or screen or setup of an instrument, the search for a new system/setup tries to minimize the difference between the old and new situation (unless it is expressly intended to improve the measurement, i.e., to reduce the uncertainty or increase the reliability).

2. In the case of undesired significant differences between the old and new system (based on the prior research referred to in (1), which may include non-climatic parallel measurements), the Tactical Meeting on Observations (TMO) will decide whether or not to pursue the change. Continuing such a change is only permitted if the prior examination referred to in (1) indicates the measurements of the two systems can be combined with each other.

3. The new sensor/screen/setup only becomes operational after approval of the TMO. Normally, this is after the completion of the steps (1) and (2).

4. For the introduction of a new sensor/screen/setup on a national scale parallel measurements are carried at a number of locations for a period of 2 years.

5. The results of the measurements are documented in a public report. The metadata published on the internet noting the change references this report.

6. When a sensor/screen/electronics is no longer used, at least one copy (including documentation) will be retained and curated in the archive of historical instruments, unless it is not an essential modification.

7. When the need arises only the TMO can decide to deviate from above points.

\subsection{Change in algorithms/procedures, data processing systems or in the validation}

1. Change in algorithms/procedures, data processing systems, in the analysis (e.g., how averages, minimums or maximums are calculated) in the validation and any corrections and in the archiving

9 This is only waived be if there are, in the opinion of the Tactical Meeting on Observations, good reasons for it (this also applies to other cases where in this document discussed parallel measurements). 
of the data in the climate database (resolution) are preceded by research that indicates what the effect of the change is on the measurements.

2. Changes that (may) affect the homogeneity of measurement series in the climate database are to be submitted to the Tactical Meeting on Observations (TMO). The TMO decides on the steps to be taken.

3. The results of the measurements are documented in a public report. The metadata published on the internet noting the change references this report.

4. Where necessary and where possible changes are retroactively implemented in the climate database (leader: Department of Climate Data and Climate Advice).

\subsection{Defects or abnormalities in sensors or the setup during measurement}

1. Defects or abnormalities in sensors or the setup of instruments, which occurred during the measurement and were noticed during maintenance or inspection, due to validation ${ }^{10}$ or during calibration in the lab. These are documented by the maintenance, inspection, or calibration lab groups in a report indicating: (a) sensor type and serial number, (b) the location of the sensor at the time of the occurrence of the defect/deviation, (c) the nature of the defect or deviation, (d) the magnitude of the error in the measurements (as far as possible), and (e) the period wherein the sensor deviated (or an estimate thereof).

2. The report mentioned above (1) is archived in the stations metadata database.

3. The Department Infrastructure-Information Services applies retroactively the quality codes of the measurements (for the unreliable period). If possible, the measurements themselves are corrected and the QC code should be accordingly updated. ${ }^{11}$

\subsection{Changes in the setup and maintenance of the station}

1. Changes in the setup and maintenance of the station are preceded by an investigation that indicates what the effect of the change on the measurements is.

2. The Inspection Department is responsible for documentation of the compliance with agreements with regard to the setup and maintenance.

3. Changes that (may) affect the homogeneity of measurement series are submitted to the Tactical Meeting on Observations (TMO). The TMO decides on the steps to be taken.

4. The results of the measurements are documented in a public report. The metadata published on the internet noting the change references this report.

10 Validation monitors and routinely evaluates the quality and consistency of the data, including the extremes (see GCOS monitoring Principle 4 in Appendix 2). Reports are publicly available.

11 This may require a change in the procedures of the Department Infrastructure-Information Services. In the near future in the new climate database will store two values: the measured value of the variable (level I) and the value of requested corrected variable (level II), corresponding to WMO guidelines. 


\subsection{Changes in instrument maintenance}

1. Changes in the instrument maintenance are preceded by an investigation that indicates what the effect of the change is on the measurements.

2. Changes that (may) affect the homogeneity of measurement series are submitted to the TMO. The TMO decides on the steps to be taken.

3. The results of the measurements are documented in a public report. The metadata published on the internet noting the change references this report.

\subsection{Changes in calibration procedures and techniques}

1. Changes calibration procedures and techniques are preceded by an investigation that indicates what the effect of the changes is on the measurements.

2. Changes that (may) affect the homogeneity of measurement series are submitted to the TMO. The TMO decides on the steps to be taken.

3. All calibrations are based on reference standards that are traceable to the SI. Calibration certificates of the reference standards are evaluated, documented and archived.

4. The results of the measurements under (1) are documented in a public report. The metadata published on the internet noting the change references this report.

5. If a reference sensor is replaced the old one (if appropriate) is archived.

\subsection{Abrupt or slow changes in the surrounding of the station}

1. Abrupt or slow changes in the surrounding of the measurement site are documented in accordance to the inspection protocol during station visits.

2. For each station the Department of Climate Data and Climate Advice monitors trends in the ECVs compared to surrounding stations so that inhomogeneities, due to, for example, slow changes of the environment, are identified at an early stage. ${ }^{12}$

3. When the surrounding no longer meets the WMO requirements for one or more variables, this is reported in the status overview of the station.

4. If case (3) happens a recommendation will be drafted in consultation with the TMO. The TMO decides on the steps to be taken.

5. The history of the changes in the environment of the measurement site is recorded in the station metadata database in the form of photos and reports. This is made publicly available via the internet.

\subsection{Changes in the protocol for the documentation of metadata}

1. Changes in the protocol for documenting metadata are reviewed and adopted by the TMO. This assessment focuses on the effects of the change on the possibility to monitor the quality and homogeneity of time series.

\footnotetext{
${ }^{12}$ At present, this is not standard practice at KNMI. The Department of Climate Data and Climate Advice will develop a methodology for this. The aim is to automate the method and then transfer it to the Department Infrastructure-Information Services.
} 


\section{Literature}

Conrad, V. and LD Pollak. 1962. Methods in Climatology. Harvard Univ. Press, Cambridge, Mass., $459 \mathrm{pp}$.

KNMI, 2000. Handbook Observations. KNMI, De Bilt (in English).

Wessels, HRA 2005. Protocol change meetinfrastrucuur, procedures change in metering infrastructure, particularly in measurements used for climatological purposes. Technical report 278, KNMI, De Bilt.

WMO, 2010. Implementation Plan for the Global Observing System for Climate in Support of the UNFCCC, 2010 update. WMO-TD / No 1523.

WMO. Technical Regulations. WMO-No.49 (3 volumes).

WMO, 1996. Guide to Meteorological Instruments and Methods of Observation. WMO No.8, 6th Ed., Geneva.

WMO, 1981. Manual on the Global Observing System. WMO 544, Geneva.

WMO, 2010. Guide to Climatological Practices. WMO Nr.100, Geneva (3rd Edition). 


\section{Appendix 1: GCOS Essential Climate Variables}

\begin{tabular}{|c|c|}
\hline Domain & GCOS Essential Climate Variables \\
\hline $\begin{array}{l}\text { Atmospheric } \\
\text { (over land, sea } \\
\text { and ice) }\end{array}$ & $\begin{array}{l}\text { Surface [a]: Air temperature, Wind speed and direction, } \\
\text { Water vapour, Pressure, Precipitation, Surface radiation } \\
\text { budget. } \\
\text { Upper-air [b]: Temperature, Wind speed and direction, Water } \\
\text { vapour, Cloud properties, Earth radiation budget (including } \\
\text { solar irradiance). } \\
\text { Composition: Carbon dioxide, Methane, and other long-lived } \\
\text { greenhouse gases [c], Ozone and Aerosol, supported by their } \\
\text { precursors [d]. }\end{array}$ \\
\hline Oceanic & $\begin{array}{l}\text { Surface [e]: Sea-surface temperature, Sea-surface salinity, } \\
\text { Sea level, Sea state, Sea ice, Surface current, Ocean colour, } \\
\text { Carbon dioxide partial pressure, Ocean acidity, Phytoplankton. } \\
\text { Sub-surface: Temperature, Salinity, Current, Nutrients, } \\
\text { Carbon dioxide partial pressure, Ocean acidity, Oxygen, } \\
\text { Tracers. }\end{array}$ \\
\hline Terrestrial & $\begin{array}{l}\text { River discharge, Water use, Groundwater, Lakes, Snow cover, Glaciers and ice } \\
\text { caps, Ice sheets, Permafrost, Albedo, Land cover (including vegetation type), } \\
\text { Fraction of Absorbed Photosynthetically Active Radiation (FAPAR), Leaf Area } \\
\text { Index (LAI), Above-ground biomass, Soil carbon, Fire disturbance, Soil moisture. }\end{array}$ \\
\hline
\end{tabular}

[a] Including measurements at standardized, but globally varying heights in close proximity to the surface.

[b] Up to the stratopause.

[c] Including nitrous oxide $\left(\mathrm{N}_{2} \mathrm{O}\right)$, chlorofluorocarbons (CFCs), hydrochlorofluorocarbons (HCFCs), hydrofluorocarbons (HFCs), sulphur hexafluoride $\left(\mathrm{SF}_{6}\right)$, and perfluorocarbons (PFCs).

[d] In particular nitrogen dioxide $\left(\mathrm{NO}_{2}\right)$, sulphur dioxide $\left(\mathrm{SO}_{2}\right)$, formaldehyde $(\mathrm{HCHO})$ and carbon monoxide (CO).

[e] Including measurements within the surface mixed layer, usually within the upper $15 \mathrm{~m}$. 


\title{
Appendix 2 GCOS Climate Monitoring Principles
}

\author{
Effective monitoring systems for climate should adhere to the following principles. ${ }^{13}$
}

1. The impact of new systems or changes to existing systems should be assessed prior to implementation.

2. A suitable period of overlap for new and old observing systems should be required.

3. The results of calibration, validation and data homogeneity assessments, and assessments of algorithm changes, should be treated with the same care as data.

4. A capacity to routinely assess the quality and homogeneity of data on extreme events, including high-resolution data and related descriptive information, should be ensured.

5. Consideration of environmental climate-monitoring products and assessments, such as IPCC assessments, should be integrated into national, regional and global observing priorities.

6. Uninterrupted station operations and observing systems should be maintained.

7. A high priority should be given to additional observations in data-poor regions and regions sensitive to change.

8. Long-term requirements should be specified to network designers, operators and instrument engineers at the outset of new system design and implementation.

9. The carefully-planned conversion of research observing systems to long-term operations should be promoted.

10. Data management systems that facilitate access, use and interpretation should be included as essential elements of climate monitoring systems.

Furthermore, satellite systems for monitoring climate need to:

(a) Take steps to make radiance calibration, calibration-monitoring and satellite-to-satellite crosscalibration of the full operational constellation a part of the operational satellite system; and

(b) Take steps to sample the Earth system in such a way that climate-relevant (diurnal, seasonal, and long-term interannual) changes can be resolved.

Thus satellite systems for climate monitoring should adhere to the following specific principles:

11. Constant sampling within the diurnal cycle (minimizing the effects of orbital decay and orbit drift) should be maintained.

12. A suitable period of overlap for new and old satellite systems should be ensured for a period adequate to determine inter-satellite biases and maintain the homogeneity and consistency of time-series observations.

13. Continuity of satellite measurements (i.e., elimination of gaps in the long-term record) through appropriate launch and orbital strategies should be ensured.

14. Rigorous pre-launch instrument characterization and calibration, including radiance confirmation against an international radiance scale provided by a national metrology institute, should be ensured.

\footnotetext{
${ }^{13}$ The basic principles were adopted by the Conference of the Parties (COP) to the United Nations Framework Convention on Climate Change (UNFCCC) through decision 5 / CP.5 at COP 5 in November 1999. The complete set of principles was is adopted by the Congress of the World Meteorological Organization (WMO) through Resolution 9 (Cg-XIV) in May 2003; agreed by the Committee on Earth Observation Satellites (CEOS) at its 17th Plenary in November 2003; and is adopted by COP through decision 11 / CP.9 at COP 9 in December 2003.
} 
15. On-board calibration adequate for climate system observations should be ensured and associated instrument characteristics monitored.

16. Operational production of priority climate products should be sustained and peer-reviewed new products should be introduced as appropriate.

17. Data systems needed to facilitate user access to climate products, metadata and raw data, including key data for delayed-mode analysis, should be established and maintained.

18. Use of functioning baseline instruments that meet the calibration and stability requirements stated above should be maintained for as long as possible, even when these exist on decommissioned satellites.

19. Complementary in situ baseline observations for satellite measurements should be maintained through appropriate activities and cooperation.

20. Random errors and time-dependent biases in satellite observations and derived products should be identified. 


\section{Appendix 3: Abbreviations}

$\begin{array}{ll}\text { AWS } & \text { Automatic Weather Station } \\ \text { ECV } & \text { Essential Climate Variable } \\ \text { GCOS } & \text { Global Climate Observing System } \\ \text { PMIC } & \text { Protocol Measurement Infrastructure Changes } \\ \text { SI } & \text { International System of units } \\ \text { TMO } & \text { Tactical Meeting on Observations } \\ \text { TR } & \text { Technical Report } \\ \text { WMO } & \text { World Meteorological Organization }\end{array}$




\section{APPENDIX B \\ Glossary}

Annual homogenisation: Homogenisation using annual data (averages or sums). May also include the magnitude of the seasonal cycle.

ANOVA: A joint correction method computing all corrections of a network simultaneously assuming all have the same regional climate signal and breaks are a step function. The method minimizes the noise using a least square method and as such the equations are the same as those of the statistical test for differences in means called ANalysis Of VAriance.

Benchmarking: Performance testing of homogenisation methods using realistic open data, which typically involves multiple methods, contributions or operators; it is a community effort. As such benchmarking is more than just a validation of a method.

Corrections: Adjustments made to raw observations aiming to make them more homogeneous. Together all corrections can also be called the estimated break signal in a multiple breakpoint setting. A corrected value is naturally not exactly right, in science we quantify how accurately we know a value.

Daily homogenisation: Homogenisation using daily data (averages or sums). In case of daily homogenisation the default is to consider all days serially in one long time series.

Joint methods: Homogenisation methods that jointly detection or correction all inhomogeneities in multiple stations in the same step.

Monthly homogenisation: Homogenisation using monthly data (averages or sums). This can be done with 12 monthly time series in parallel (default) or with all months considered serially in one long time series. Often in combination with annual homogenisation.

Multiple breakpoint methods: Homogenisation methods that detection or correction multiple inhomogeneities in one station/time series in the same step.

Seasonal homogenisation: Homogenisation using seasonal data (averages or sums). This can be done with 12 monthly time series in parallel (default) or with all months considered serially in one long time series. Often in combination with annual homogenisation.

SNHT: Standard Normal Homogeneity Test. 


\section{References}

Aguilar E., I. Auer, M. Brunet, T.C. Peterson and J. Wieringa. Guidelines on climate metadata and homogenization. World Meteorological Organization, WMO-TD No. 1186, WCDMP No. 53, Geneva, Switzerland, 55 p., 2003.

Alexandersson, A., 1986: A homogeneity test applied to precipitation data. Journal of Climatology, 6, pp. 661-675. https://doi.org/10.1002/joc.3370060607

Alexandersson, H. and A. Moberg, 1997: Homogenization of Swedish temperature data .1. Homogeneity test for linear trends. International Journal of Climatology, 17, pp. 25-34. https://doi.org/10.1002/(SICI)1097-0088(199701)17:1<25::AID-JOC103>3.0.CO;2-J

Auchmann, R. and S. Brönnimann, 2012: A physics-based correction model for homogenizing subdaily temperature series. Journal of Geophysical Research, 117: D17119. https://doi.org/10.1002/joc.3370060607

Azorin-Molina, C., S.M. Vicente-Serrano, T.R. McVicar, S. Jerez, A. Sanchez-Lorenzo, J. López-Moreno, J. Revuelto, R.M. Trigo, J.A. Lopez-Bustins and F. Espírito-Santo, 2014: Homogenization and Assessment of Observed Near-Surface Wind Speed Trends over Spain and Portugal, 1961-2011. Journal of Climate, 27, pp. 3692-3712. https://doi.org/10.1175/JCLI-D-13-00652.1

Begert M., Schlegel T. and Kirchhofer W., 2005: Homogeneous temperature and precipitation series of Switzerland from 1864 to 2000. International Journal of Climatology, 25, pp. 65-80. https://dx.doi.org/10.1002/ioc.1118

Böhm, R., Jones, P.D., Hiebl, J., Frank, D., Brunetti, M. and Maugeri, M., 2010: The early instrumental warm-bias: a solution for long central European temperature series 1760-2007. Climatic Change, 101, pp. 41-67. https://doi.org/10.1007/s10584-009-9649-4

Brückner, E., 1890: Klimaschwankungen seit 1700 nebst Bemerkungen über Klimaschwankungen der Diluvialzeit. E.D. Hölzel, Wien and Olnütz.

Brunet, M., Oscar Saladié, Phil Jones, Javier Sigró, Enric Aguilar, Anders Moberg, David Lister, Alexander Walther, Diego Lopez and Carlos Almarza, 2006: The development of a new dataset of Spanish daily adjusted temperature series (SDATS). International Journal of Climatology, 26, pp. 1777-1802. https://doi.org/10.1002/joc.1338

Brunet, M., J. Asin, J. Sigró, M. Bañón, F. García, E. Aguilar, J. Esteban Palenzuela, T.C. Peterson, P. Jones, 2011: The minimization of the screen bias from ancient Western Mediterranean air temperature records: an exploratory statistical analysis. International Journal Climatology, 31, pp. 1879-1895. https://doi.org/10.1002/joc.2192

Brogniez, H., English, S., Mahfouf, J.-F., Behrendt, A., Berg, W., Boukabara, S., Buehler, S. A., Chambon, P., Gambacorta, A., Geer, A., Ingram, W., Kursinski, E. R., Matricardi, M., Odintsova, T. A., Payne, V. H., Thorne, P. W., Tretyakov, M. Yu. and Wang, J., 2016: A review of sources of systematic errors and uncertainties in observations and simulations at $183 \mathrm{GHz}$. Atmospheric Measurement Techniques, 9, pp. 2207-2221. https://doi.org/10.5194/amt-9-2207-2016

Brunetti M., Maugeri, M., Monti, F. and Nanni, T., 2006: Temperature and precipitation variability in Italy in the last two centuries from homogenized instrumental time series. International Journal of Climatology, 26, pp. 345-381. https://doi.org/10.1002/joc.1251

Caussinus, H. and Mestre, O., 2004: Detection and correction of artificial shifts in climate series. Journal of the Royal Statistical Society, Series C (Applied Statistics), 53, pp. 405-425. https://doi.org/10.1111/j.1467-9876.2004.05155.x

Conrad, V. and C. Pollak, 1950: Methods in Climatology. Harvard University Press, Cambridge, MA, $459 \mathrm{p}$.

Conrad, V., 1925: Homogenitätsbestimmung meteorologischer Beobachtungsreihen. Meteorologische Zeitschrift, 482-485.

Conrad V., 1944: Methods in Climatology. Harvard University Press, 228 p. 
Conrad V. and O. Schreier, 1927: Die Anwendung des Abbe'schen Kriteriums auf physikalische Beobachtungsreihen. Gerland's Beiträge zur Geophysik, XVII, 372.

Costa, AC, and A Soares., 2009: Homogenization of Climate Data: Review and New Perspectives Using Geostatistics. Mathematical Geosciences, 41: 3, pp. 291-305. https://doi.org/10.1007/s11004008-9203-3

Cowtan, K. , Rohde, R. and Hausfather, Z., 2018: Evaluating biases in Sea Surface Temperature records using coastal weather stations. Quarterly Journal of the Royal Meteorological Society, published online. https://doi.org/10.1002/aj.3235

Cook, B.I., S.P. Shukla, M.J. Puma and L.S. Nazarenko, 2014: Irrigation as an historical climate forcing. Climate Dynamics, 44: 1715. https://doi.org/10.1007/s00382-014-2204-7

Craddock, J. M., 1979: Methods of comparing annual rainfall records for climatic purposes. Weather, 34, pp. 332-346. https://doi.org/10.1002/j.1477-8696.1979.tb03465.x

Dai, A., J. Wang, P.W. Thorne, D.E. Parker, L. Haimberger and X.L. Wang, 2011: A New Approach to Homogenize Daily Radiosonde Humidity Data. J. Clim., 24, 965-991. https://doi.org/10.1175/2010JCLI3816.1

Degaetano, A.T., 2000: A Serially Complete Simulated Observation Time Metadata File for U.S. Daily Historical Climatology Network Stations. Bulletin of the American Meteorological Society, 81: 1, pp.49-68. https://doi.org/10.1175/1520-0477(2000)081<0049:ASCSOT>2.3.CO;2

Della-Marta, P.M. and H. Wanner, 2006: A method of homogenizing the extremes and mean of daily temperature measurements. Journal of Climate, 19, pp. 4179-4197. https://doi.org/10.1175/JCLI3855.1

Domonkos, P., V. Venema and O. Mestre, 2013: Efficiencies of homogenisation methods: our present knowledge and its limitation. Proceedings of the Seventh seminar for homogenization and quality control in climatological databases, Budapest, Hungary, 24- 28 October 2011, WMO report, Climate data and monitoring, WCDMP-No. 78, pp. 11-24.

Domonkos, P., 2011: Efficiency evaluation for detecting inhomogeneities by objective homogenization methods. Theoretical and Applied Climatology, 105, pp. 455-467. https://doi.org/10.1007/s00704-011-0399-7

Domonkos, P. and Coll, J. 2016. Homogenisation of temperature and precipitation time series with ACMANT3: Method description and efficiency tests. International Journal of Climatology, 37: 4. https://doi.org/10.1002/joc.4822

Domonkos, P., V. Venema and O. Mestre, 2013: Efficiencies of homogenisation methods: our present knowledge and its limitation. Proceedings of the Seventh seminar for homogenization and quality control in climatological databases, Budapest, Hungary, 24 - 28 October 2011, WMO report, Climate data and monitoring, WCDMP-No. 78, pp. 11-24.

Dunn, R. and P. Thorne, 2017: Towards an integrated set of surface meteorological observations for climate science and applications. Proceedings from the 19th EGU General Assembly conference, Vienna, Austria.

Dunn, R.J.H., K.M. Willett, P.W. Thorne, E.V. Woolley, I. Durre, A. Dai, D.E. Parker and R.S. Vose: HadISD: a quality-controlled global synoptic report database for selected variables at long-term stations from 1973-2011. Climate of the Past, 8, pp. 1649-1679. https://doi.org/10.5194/cp-81649-2012

Gubler, S., Hunziker, S., Begert, M., Croci-Maspoli, M., Konzelmann, T., Brönnimann, S., Schwierz, C., Oria, C. and Rosas, G., 2017: The influence of station density on climate data homogenization. International Journal of Climatology, 37, pp. 4670-4683. https://doi.org/10.1002/joc.5114

Guijarro, J., 2018: Package 'climatol'. Version 3.1.1. https://CRAN.R-project.org/package=climatol and http://www.climatol.eu/

Haimberger, L., 2007: Homogenization of radiosonde temperature time series using innovation statistics. Journal of Climate, 20(7): pp. 1377-1403. https://doi.org/10.1175/JCLI4050.1 
Haimberger, L., C. Tavolato and S. Sperka, 2012: Homogenization of the global radiosonde dataset through combined comparison with reanalysis background series and neighboring stations.

Journal of Climate, 25, pp. 8108- 8131. https://doi.org/10.1175/JCLI-D-11-00668.1

Hann, J., 1880: Untersuchungen über die Regenverhältnisse von Österreich-Ungarn. II. Veränderlichkeit der Monats- und Jahresmengen. S.-B. Akad. Wiss. Wien.

Heidke P., 1923: Quantitative Begriffsbestimmung homogener Temperatur- und Niederschlagsreihen. Meteorologische Zeitschrift, pp. 114-115.

Helmert F.R., 1907: Die Ausgleichrechnung nach der Methode der kleinsten Quadrate. 2. Auflage, Teubner Verlag.

Hungarian Meteorological Service (HMS), 1996: Proceedings of the First Seminar for Homogenization of Surface Climatological Data, Budapest, Hungary, 6-12 October 1996, 44 p.

Huang, B., V. F. Banzon, E. Freeman, J. Lawrimore, W. Liu, T.C. Peterson, T.M. Smith, P.W. Thorne, S.D. Woodruff and H.-M. Zhang, 2015: Extended Reconstructed Sea Surface Temperature Version 4 (ERSST.v4). Part I: Upgrades and Intercomparisons. Journal of Climate, 28, pp. 911-930. https://doi.org/10.1175/JCLI-D-14-00006.1

Jones, P. D., D. H. Lister, T. J. Osborn, C. Harpham, M. Salmon and C. P. Morice, 2012: Hemispheric and large-scale land-surface air temperature variations: An extensive revision and an update to 2010. Journal of Geophysical Research, 117: D05127. https://doi.org/10.1029/2011JD017139

Jovanovic, B., R. Smalley, B. Timbal and S. Siems, 2017: Homogenized monthly upper-air temperature data set for Australia. International Journal of Climatology, 37, pp. 3209-3222. https://doi.org/10.1002/joc/4909

Karl, T.R., C.N. Williams Jr., P.J. Young and W.M. Wendland, 1986: A Model to Estimate the Time of Observation Bias Associated with Monthly Mean Maximum, Minimum and Mean Temperatures for the United States. Journal of Applied Meteorology, 25: 2, pp. 145-160.

https://doi.org/10.1175/1520-0450(1986)025<0145:AMTETT>2.0.CO;2

Kennedy, J.J., N.A. Rayner, R.O. Smith, D.E. Parker and M. Saunby, 2011: Reassessing biases and other uncertainties in sea surface temperature observations measured in situ since 1850: 1.

Measurement and sampling uncertainties. Journal of Geophysical Research, 116: D14103. https://doi.org/10.1029/2010JD015218

Kent, E., J. Kennedy, T. Smith, S. Hirahara, B. Huang, A. Kaplan, D. Parker, C. Atkinson, D. Berry, G. Carella, Y. Fukuda, M. Ishii, P. Jones, F. Lindgren, C. Merchant, S. Morak-Bozzo, N. Rayner, V. Venema, S. Yasui and H. Zhang, 2016: A call for new approaches to quantifying biases in observations of sea-surface temperature. Bulletin of the American Meteorological Society, 98, pp. 1601-1616. https://doi.org/10.1175/BAMS-D-15-00251.1

Killick, R., 2016: Benchmarking the Performance of Homogenisation Algorithms on Daily Temperature Data. PhD thesis, Department of Mathematics, University of Exeter, UK. http://hdl.handle.net/10871/23095

k.k. Hof- und Staatsdruckerei, 1873: Bericht über die Verhandlungen des internationalen Meteorologen-Congresses zu Wien, 2.-10. September 1873, Protokolle und Beilagen.

k.k. Zentralanstalt für Meteorologie und Geodynamik. 1906: Bericht über die internationale meteorologische Direktorenkonferenz in Innsbruck, September 1905. Anhang zum Jahrbuch 1905. k.k. Hof-und Staatsdruckerei.

Kohler M.A., 1949: Double-mass analysis for testing the consistency of records and for making adjustments. Bulletin of the American Meteorological Society, 30, pp. 188-189. https://doi.org/10.1175/1520-0477-30.5.188

Kuglitsch, F. G., R. Auchmann, R. Bleisch, S. Brönnimann, O. Martius, and M. Stewart, 2012: Break detection of annual Swiss temperature series. Journal of Geophysical Research, 117: D13105. https://doi.org/10.1029/2012JD017729

Kreil K., 1854a: Mehrjährige Beobachtungen in Wien vom Jahre 1775 bis 1850. Jahrbücher der k.k. Central-Anstalt für Meteorologie und Erdmagnetismus. I. Band - Jg 1848 und 1849, pp. 35-74. 
Kreil K., 1854b: Mehrjährige Beobachtungen in Mailand vom Jahre 1763 bis 1850. Jahrbücher der k.k. Central-Anstalt für Meteorologie und Erdmagnetismus. I. Band - Jg 1848 und 1849, pp. 75-114.

Leeper, Ronald D., Jared Rennie and Michael A. Palecki, 2015: Observational Perspectives from U.S. Climate Reference Network (USCRN) and Cooperative Observer Program (COOP) Network: Temperature and Precipitation Comparison. Journal Atmospheric and Oceanic Technology, 32, pp. 703-721. https://doi.org/10.1175/JTECH-D-14-00172.1

Li, Yingbo, Robert Lund and Anuradha Hewaarachchi, 2017: Multiple Changepoint Detection with Partial Information on Changepoint Times. ArXiv manuscript. https://arxiv.org/abs/1511.07238

Lindau, R. and V. Venema, 2016: The uncertainty of break positions detected by homogenization algorithms in climate records. International Journal of Climatology, 36, no. 2, pp. 576-589. https://doi.org/10.1002/joc.4366

Lund, R. and J. Reeves, 2002: Detection of undocumented changepoints: A revision of the two-phase regression model. Journal of Climate, 15, pp. 2547-2554.

Mekis, É. and V., Lucie A., 2011: An Overview of the Second Generation Adjusted Daily Precipitation Dataset for Trend Analysis in Canada. Atmosphere-Ocean, 49: 2, pp. 163-177.

https://doi.org/10.1080/07055900.2011.583910

Menne, M. J., C. N. Williams Jr. and M. A. Palecki, 2010: On the reliability of the U.S. surface temperature record. Journal of Geophysical Research, 115: D11108. doi:

https://doi.org/10.1029/2009JD013094

Menne, M.J. and Williams, C.N. Jr., 2009: Homogenization of temperature series via pairwise comparisons. Journal of Climate, 22:7, pp. 1700-1717. https://doi.org/10.1175/2008JCLI2263.1

Menne, M. J., Williams, C. N. Jr. and Vose, R. S., 2009: The U.S. historical climatology network monthly temperature data, version 2. Bulletin of the American Meteorological Society, 90, pp. 993-1007. https://doi.org/10.1175/2008BAMS2613.1

Mestre O., 1999: Step-by-step procedures for choosing a model with change-points. In Proceedings of the second seminar for homogenisation of surface climatological data, Budapest, Hungary, WCDMP-No.41, WMO-TD No.962, pp. 15-26.

Mestre, O., C. Gruber, C. Prieur, H. Caussinus and S. Jourdain, 2011: SPLIDHOM: A Method for Homogenization of Daily Temperature Observations. Journal of Applied Meteorology and Climatology, 50, pp. 2343-2358. https://doi.org/10.1175/2011JAMC2641.1

Minola, L., C. Azorin-Molina and D. Chen, 2016: Homogenization and Assessment of Observed NearSurface Wind Speed Trends across Sweden, 1956-2013. Journal of Climate, 29, pp. 7397-7415. https://doi.org/10.1175/JCLI-D-15-0636.1

OMSZ, 2001: Third Seminar for Homogenization and Quality Control in climatological Databases, Budapest.

Peterson T.C., D.R. Easterling, T.R. Karl, P. Groisman, N. Nicholls, N. Plummer, S. Torok, I. Auer, R. Boehm, D. Gullett, L. Vincent, R. Heino, H. Tuomenvirta, O. Mestre, T. Szentimrey, J. Salinger, E.J. Forland, I. Hanssen-Bauer, H. Alexandersson, P. Jones and D. Parker, 1998: Homogeneity adjustments of in situ atmospheric climate data: A review. International Journal of Climatology, 18, pp. 1493-1517. https://doi.org/10.1002/(SICI)1097-0088(19981115)18:13\%3C1493::AIDJOC329\%3E3.0.CO;2-T

Petrovic, P., 2004: Detecting of inhomogeneities in time series using Real Precision Method. In: Fourth seminar for homogenization and quality control in climatological databases, WCDMP-No. 56, WMO-TD No. 1236, WMO, Geneva.

Quayle, R.G., D.R. Easterling, T.R. Karl and P.Y. Hughes, 1991: Effects of recent thermometer changes in the cooperative station network. Bulletin of the American Meteorological Society, 72, pp. 17181723. https://doi.org/10.1175/1520-0477(1991)072\%3C1718:EORTCI\%3E2.0.CO;2

Reeves, J., J. Chen, X. L. Wang, R. Lund and Q. Lu, 2007: A Review and Comparison of Changepoint Detection Techniques for Climate Data. Journal of Applied Meteorology and Climatology, 46, pp. 900-915. https://doi.org/10.1175/JAM2493.1 
Rennie, Jared, Jay Lawrimore, Byron Gleason, Peter Thorne, Colin Morice, Matthew Menne, Claude Williams, Waldenio Gambi de Almeida, John Christy, Meaghan Flannery, Masahito Ishihara, Kenji Kamiguchi, Abert Klein Tank, Albert Mhanda, David Lister, Vyacheslav Razuvaev, Madeleine Renom, Matilde Rusticucci, Jeremy Tandy, Steven Worley, Victor Venema, William Angel, Manola Brunet, Bob Dattore, Howard Diamond, Matthew Lazzara, Frank Le Blancq, Juerg Luterbacher, Hermann Maechel, Jayashree Revadekar, Russell Vose and Xungang Yin, 2014: The International Surface Temperature Initiative global land surface databank: monthly temperature data version 1 release description and methods. Geoscience Data Journal, 1, pp. 75-102.

https://doi.org/10.1002/gdj3.8

Ribeiro, S., J. Caineta, A.C. Costa and R. Henriques, 2017: GSIMCLI: a geostatistical procedure for the homogenisation of climatic time series. International Journal of Climatology, 37:8, pp. 3452-3467. https://doi.org/10.1002/joc.4929

Rienzner, M. and C Gandolfi, 2013: A procedure for the detection of undocumented multiple abrupt changes in the mean value of daily temperature time series of a regional network. International Journal of Climatology, 33, pp. 1107-1120. https://doi.org/10.1002/joc.3496

Rohde R., R. Muller, R. Jacobsen, S. Perlmutter, A. Rosenfeld, et al., 2013: Berkeley Earth Temperature Averaging Process. Geoinformatics \& Geostatistics: An Overview 1:2. https://doi.org/10.4172/2327-4581.1000103

Schröder, M., M. Lockhoff, J. Forsythe, H. Cronk, T. Vonder Haar and R. Bennartz, 2016: The GEWEX Water Vapor Assessment: Results from Intercomparison, Trend, and Homogeneity Analysis of Total Column Water Vapor. Journal of Applied Meteorology and Climatology, 55, pp. 1633-1649. https://doi.org/10.1175/JAMC-D-15-0304.1

Štěpánek, P., Zahradníček, P., and Skalák, P.: Data quality control and homogenization of the air temperature and precipitation series in the Czech Republic in the period 1961-2007. Advances in Science and Research, 3, pp. 23-26, 2009. https://doi.org/10.5194/asr-3-23-2009

Szentimrey, T., 1999: Multiple Analysis of Series for Homogenization (MASH). Proceedings of the second seminar for homogenization of surface climatological data, Budapest, Hungary; WMO, WCDMP-No. 41, 27-46.

Szentimrey, T., 2007: Manual of homogenization software MASHv3.02. Hungarian Meteorological Service, $65 \mathrm{p}$.

Szentimrey, T., 2008: Development of MASH homogenization procedure for daily data. Proceedings of the fifth seminar for homogenization and quality control in climatological databases. Budapest, Hungary, 2006; WCDMP-No. 71, pp. 123-130.

Szentimrey, T., 2018: New version MASHv4.01 for joint homogenization of mean and standard deviation. EMS Annual Meeting: European Conference for Applied Meteorology and Climatology 2018, Budapest, Hungary, no. EMS2018-331.

Thorne, P.W., K.M. Willett, R.J. Allan, S. Bojinski, J.R. Christy, N. Fox, S. Gilbert, I. Jolliffe, J.J. Kennedy, E. Kent, A. Klein Tank, J. Lawrimore, D.E. Parker, N. Rayner, A. Simmons, L. Song, P.A. Stott and B. Trewin, 2011: Guiding the Creation of A Comprehensive Surface Temperature Resource for Twenty-First-Century Climate Science. Bulletin of the American Meteorological Society, 92, ES40ES47. https://doi.org/10.1175/2011BAMS3124.1

Toreti A., F.G. Kuglitsch, E. Xoplaki, J. Luterbacher and H. Wanner, 2010. A Novel Method for the Homogenization of Daily Temperature Series and Its Relevance for Climate Change Analysis. Journal of Climate, 23, pp. 5325-5331. https://doi.org/10.1175/2010JCLI3499.1

Toreti, Andrea, Franz G. Kuglitsch, Elena Xoplaki, Jürg Luterbacher, 2012: A Novel Approach for the Detection of Inhomogeneities Affecting Climate Time Series. Journal of Applied Meteorology and Climatology, 51, 317-326. https://doi.org/10.1175/JAMC-D-10-05033.1

Trewin, B, 2013. A daily homogenized temperature data set for Australia. International Journal of Climatology, 33: 6, pp. 1510-1529. https://doi.org/10.1002/joc.3530 
Trewin, B. C. and A. C. F. Trevitt, 1996: The development of composite temperature records. International Journal of Climatology, 16, pp. 1227-1242. https://doi.org/10.1002/(SICI)10970088(199611)16:11\%3C1227::AID-JOC82\%3E3.0.CO;2-P

Trewin, B.C., 2012: Techniques involved in developing the Australian Climate Observations Network Surface Air Temperature (ACORN-SAT) dataset. CAWCR Technical Report 49, Centre for Australian Weather and Climate Research, Melbourne. http://cawcr.gov.au/technical-reports/CTR 049.pdf

Trewin B.C., 2013: A daily homogenized temperature data set for Australia. International Journal of Climatology, 33, 1510-1529. https://doi.org/10.1002/joc.3530

Venema, V.K.C., O. Mestre, E. Aguilar, I. Auer, J.A. Guijarro, P. Domonkos, G. Vertacnik, T. Szentimrey, P. Stepanek and coauthors, 2012: Benchmarking homogenization algorithms for monthly data. Climate of the Past, 8, pp. 89-115. https://doi.org/10.5194/cp-8-89-2012

Vose, Russell S., Claude N. Williams Jr., Thomas C. Peterson, Thomas R. Karl and David R. Easterling, 2003: An evaluation of the time of observation bias adjustment in the U.S. Historical Climatology Network. Geophysical Research Letters, 30: 20. https://doi.org/10.1029/2003GL018111

Vincent, L.A., X.L. Wang, E.J. Milewska, H. Wan, F. Yang and V. Swail, 2012: A second generation of homogenized Canadian monthly surface air temperature for climate trend analysis. Journal of Geophysical Research - Atmosphere, 117: D18110. https://doi.org/10.1029/2012JD017859

Vincent, L.A., E. Milewska, R. Hokinson and L. Malone, 2009: Bias in minimum temperature introduced by a redefinition of the climatological day at the Canadian Synoptic stations. Journal of Applied Meteorology and Climatology, 48, pp. 2160-2168. https://doi.org/10.1175/2009JAMC2191.1

Vincent, L. A., 1998: A technique for the identification of inhomogeneities in Canadian temperature series. Journal of Climate, 11, pp. 1094-1104. https://doi.org/10.1175/15200442(1998)011<1094:ATFTIO>2.0.CO;2

Vose, R.S., C.N. Williams Jr., T.C. Peterson, T.R. Karl and D.R. Easterling, 2003: An evaluation of the time of observation bias adjustment in the U.S. Historical Climatology Network. Geophysical Research Letters, 30, no. 20, 2046. https://doi.org/10.1029/2003GL018111

Wan, H., X.L. Wang, and V.R. Swail, 2010: Homogenization and Trend Analysis of Canadian NearSurface Wind Speeds. Journal of Climate, 23, pp. 1209-1225. https://doi.org/10.1175/2009JCLI3200.1

Wang, X.L., H. Xu, B. Qian, Y. Feng and E. Mekis, 2017: Adjusted daily rainfall and snowfall data for Canada. Atmosphere-Ocean, 55: 3, pp. 155-168. https://doi.org/10.1080/07055900.2017.1342163

Wang, X. L. and Y. Feng, 2013: RHtestsV4 User Manual. Climate Research Division, Atmospheric Science and Technology Directorate, Science and Technology Branch, Environment Canada. , published online July 2013, 28 p. http://etccdi.pacificclimate.org/software.shtml

Wang, X., H. Chen, Y. Wu, Y. Feng and Q. Pu, 2010: New Techniques for the Detection and Adjustment of Shifts in Daily Precipitation Data Series. Journal of Applied Meteorology and Climatology, 49, pp. 2416-2436. https://doi.org/10.1175/2010JAMC2376.1

Wang, X.L., Y. Feng and L.A. Vincent, 2013: Observed changes in one-in-20 year extremes of Canadian surface air temperatures. Atmosphere-Ocean, 52, pp. 222-231. https://doi.org/10.1080/07055900.2013.818526

Wang, X. L., 2008a: Accounting for autocorrelation in detecting mean-shifts in climate data series using the penalized maximal $t$ or $F$ test. Journal of Applied Meteorology and Climatology, 47, pp. 2423-2444. https://doi.org/10.1175/2008JAMC1741.1

Wang, X. L., 2008b: Penalized maximal $F$ test for detecting undocumented mean-shift without trend change. Journal of Atmospheric and Oceanic Technology, 25, pp. 368-384 https://doi.org/10.1175/2007/JTECHA982.1 
Wang, X.L., Q.H. Wen, and Y. Wu, 2007: Penalized Maximal $t$ Test for Detecting Undocumented Mean Change in Climate Data Series. Journal of Applied Meteorology and Climatology, 46, pp. 916-931. https://doi.org/10.1175/JAM2504.1

Wang, X.L., 2003: Comments on "Detection of Undocumented Changepoints: A Revision of the TwoPhase Regression Model". Journal of Climate, 16, pp. 3383-3385. https://doi.org/10.1175/15200442(2003)016<3383: CODOUC>2.0.CO;2

Wen, Q. H., X.L. Wang and A. Wong, 2011: A hybrid-domain approach to modeling climate data time series. Journal of Geophysical Research - Atmospheres, 116, D18112. https://doi.org/10.1029/2011JD015850

Willett, Kate M., Claude N. Williams, Ian Jolliffe, Robert Lund, Lisa Alexander, Stefan Bronnimann, Lucie A. Vincent, Steve Easterbrook, Victor Venema, David Berry, Rachel Warren, Giuseppina Lopardo, Renate Auchmann, Enric Aguilar, Matthew Menne, Colin Gallagher, Zeke Hausfather, Thordis Thorarinsdottir, and Peter W. Thorne, 2014: A framework for benchmarking of homogenisation algorithm performance on the global scale. Geoscientific Instrumentation, Methods and Data Systems, 3, pp. 187-200. https://doi.org/10.5194/gi-3-187-2014

Winkler, P., 2009: Revision and necessary correction of the long-term temperature series of Hohenpeissenberg. 1781-2006. Theoretical and Applied Climatology, 98: 259.

https://doi.org/10.1007/s00704-009-0108-y

World Meteorological Organization, 2011: Guide to climatological practices. WMO No. 100, World Meteorological Organization, Geneva.

Rennie, J.J. and coauthors, 2014: The international surface temperature initiative global land surface databank: monthly temperature data release description and methods. Geoscience Data Journal, 1: 2 . https://doi.org/10.1002/gdj3.8

World Meteorological Organization, 2004: Guidelines on climate data rescue. WMO/TD No. 1210, World Meteorological Organization, Geneva.

WMO, 1966: Climatic Change, Report of a working group of the Commission for Climatology. Technical Note 79, WMO - No. 195. TP.100, 79 p.

WMO, 1999: Proceedings of the Second Seminar for Homogenization of Surface Climatological Data. Budapest, Hungary, 9 - 13 November 1998, 214 p.

WMO, 2004: Fourth Seminar for Homogenization and Quality Control in Climatological Databases. Budapest, Hungary, 6-10 October 2003, WCDMP-No 56, WMO-TD No. 1236, 243 p.

WMO, 2006: Proceedings of the Fifth Seminar for Homogenization and Quality Control in Climatological Databases. Budapest, Hungary, 29 May -2 June 2006. Climate Data and Monitoring WCDMP- No 71, WMO/TD- No. 1493.

WMO, 2010: Proceedings of the Meeting of COST-ESO601 (HOME) Action, Management Committee and Working groups and Sixth Seminar for Homogenization and Quality Control in Climatological Databases. Budapest, Hungary, 26 - 30 May 2008, WMO reports on Climate Data and Monitoring, WCDMP-No. 76.

WMO, 1993: Guide on the global data-processing system. Report, WMO-No. 305, Secretariat of the World Meteorological Organization, Geneva, Switzerland.

WMO, 2017: WIGOS Metadata Standard. Report. WMO-NO 1192, World Meteorological Organization, Geneva, Switzerland.

Zhang, L., G.-Y. Ren, Y.-Y. Ren, A.-Y. Zhang, Z.-Y. Chu and Y.-Q. Zhou, 2014: Effect of data homogenisation on estimate of temperature trend: a case of Huairou station in Beijing Municipality. Theoretical and Applied Climatology, 115, pp. 365- 373.

https://doi.org/10.1007/s00704-013-0894-0 